\title{
Deformation and Failure in Extreme Regimes by High-Energy Pulsed Lasers: A Review*
}

\author{
Tane P. Remington ${ }^{1}$, Bruce A. Remington ${ }^{2}$, Eric N. Hahn ${ }^{1}$, Marc A. Meyers ${ }^{1, \mathrm{a}}$ \\ ${ }^{I}$ The University of California, San Diego, San Diego, La Jolla, CA 92093 USA \\ ${ }^{2}$ Lawrence Livermore National Laboratory, Livermore, CA 94550 USA \\ ${ }^{a}$ Corresponding author mameyers@ucsd.edu
}

\begin{abstract}
The use of high-power pulsed lasers to probe the response of materials at pressures of hundreds of $\mathrm{GPa}$ up to several $\mathrm{TPa}$, time durations of nanoseconds, and strain rates of $10^{6}-10^{10} \mathrm{~s}^{-1}$ is revealing novel mechanisms of plastic deformation, phase transformations, and even amorphization. This unique experimental tool, aided by advanced diagnostics, analysis, and characterization, allows us to explore these new regimes that simulate those encountered in the interiors of planets. Fundamental Materials Science questions such as dislocation velocity regimes, the transition between thermally-activated and phonon drag regimes, the slip-twinning transition, the ultimate tensile strength of metals, the dislocation mechanisms of void growth are being answered through this powerful tool. In parallel with experiments, molecular dynamics simulations provide modeling and visualization at comparable strain rates $\left(10^{8}-10^{10} \mathrm{~s}^{-1}\right)$ and time durations (hundreds of picoseconds). This powerful synergy is illustrated in our past and current work, using representative face-centered cubic (fcc) copper, body-centered cubic (bcc) tantalum and diamond cubic silicon as model structures.

* This paper is dedicated to Professor J.C.M. Li on his $90^{\text {th }}$ birthday. The seminal contributions of Professor Li have covered over sixty years of uninterrupted work and have advanced our understanding of the mechanical response of metals, polymers and metallic glasses in a significant manner. One of us (M.A. Meyers) had the fortune to
\end{abstract}


follow Professor Li's work since his graduate student days (1970-1974), and the stellar example of his teaching role has been a guiding light in his career.

\section{Introduction}

The importance of understanding the response of materials in extreme environments is ever more important, in view of the regimes encountered in planetary formation dynamics and in planetary interiors, including the now more than 2000 discovered exoplanets. These regimes can now be created in laboratories using high power lasers [1]. In the domain of Materials Science, there are important questions that are being successfully addressed by laser shock experiments coupled with molecular dynamics simulations. Some of these are: dislocation velocity and the possible transonic and supersonic regimes, the transition between thermally-activated and phonon drag regimes, the slip-twinning transition, the ultimate tensile strength of metals, the dislocation mechanisms of void growth.

Ever since the early experiments by the Hopkinsons, researchers have sought to understand the response of materials to rapid loading. Hopkinson used explosives and the rapid energy release rate of the chemical reactives was the early source of interest [2]. Actually, the Hopkinsons were preceded by French military engineers who conducted the famous Metz cannon ball experiments in the late 1830s [2]. Hollow spherical steel containers ( $\sim 16 \mathrm{~cm}$ diameter) were filled with explosives which were detonated. The fragments were captured in clay and their kinetic energy was correlated to the crater volume and penetration into clay. This enabled the calculation of the fragment velocities. Thus, correlations were obtained between parameters and fragment size. The Munroe charges followed, in the late $19^{\text {th }}$ century. 
Explosively-driven flyer plates have been used since World War 2 to create high pressure pulses in materials under controlled stress state and pulse duration [3]. The work of Cyril S. Smith in this respect represents a milestone. He was the first to use dislocation concepts to explain the formation of deformations structures at the shock front [4].

Several methods have been developed to study materials at high pressures, temperatures and strain rates. Some of the most widely known are the flyer plates (accelerated by explosives, exploding wires, or gas/powder guns) and direct energy deposition by explosives or lasers. Figure 1 shows three techniques that are commonly used to generate shock waves: (a) the direct application of explosives to the surface of materials with a grazing detonation, which generates a triangular shock pulse, (b) the detonation of explosives at a normal to the surface with the acceleration of a flyer plate that impacts the material simultaneously over its surface, and (c) the gas gun, in which a flyer plate mounted on a sabot is driven by compressed gas [5]. The source of the gas can be either a high-pressure tank or the deflagration of gun powder. Figure 1(a) shows shock waves generated by direct contact of the explosive with the metal; the wave travels at an oblique angle which is determined by the velocities of detonation and shock-wave propagation. In Figure 1(b) an explosive lens is used to accelerate a flyer plate that impacts the target simultaneously over its top surface and creates a wave propagating normally to the surface. In a laboratory environment gas and powder guns are often used to accelerate flyer plates. This is shown in Fig. 1(c), where the flyer plate is mounted on a sabot; this ensures good planarity and normality of the wave. The flyer plate method accelerates a metal "flyer" onto the sample creating a shock wave traveling through both in opposite directions. The resultant shock wave can be explained using simple governing 
equations: Newton's third law and the conservation of momentum, energy, and mass. The reaction force between the flyer and the target is equivalent and the impulse is proportional to the change in momentum. These equations are known as RankineHugoniot in honor of these scientists that applied them for the first time. In combination with an equation of state for the material, all shock variables (pressure, temperature, internal energy, shock and particle velocities) are defined. The thickness of the flyer determines the pulse duration. Explosively detonated flyer plates can also force the two materials together, effectively bonding them; this is known as explosive welding. This method is used for experiments that study how fast fragments are released by explosives. The gas gun configuration uses highly compressed gas (air, hydrogen, nitrogen, or combustion products) to accelerate projectiles at velocities over $7.5 \mathrm{~km} / \mathrm{s}$ (in the twostage configuration). These velocities simulate impacts of particles on spacecraft and satellite components.

The use of the gas gun technique increased the reproducibility, planarity of impact, and control of shock wave parameters in a laboratory setting. Two types of gas guns have evolved over the years: a single-stage gas gun limited to impact velocities of $\sim 1 \mathrm{~km} / \mathrm{s}$, and a two-stage gas gun with maximum velocities of $\sim 7 \mathrm{~km} / \mathrm{s}$. The duration of the shock pulses reachable by all the aforementioned methods range from 1-3 $\mu$ s for explosively-driven flyer plates to $0.1-1 \mu$ s for gas guns. Specialty techniques have also been developed but are less common, such as electromagnetically accelerated devices. Both the explosively driven and gas gun experiments require large specimens, of which only a minute portion is used in subsequent characterization. Hence, most of the material is lost. The former require special ranges and bunkers and the attachment of 
instrumentation to field experiments is complex and cumbersome. Lasers present a unique advantage of miniaturization and enable repeated experiments with relative ease of instrumentation, such as VISAR or simultaneous diffraction. The recovery is also much easier because of the small sizes.

The first functional laser became operational in 1960 (a synthetic ruby crystal was used to produce red laser light at $694 \mathrm{~nm}$ wavelength). This was accomplished under the direction of Theodore H. Maiman at Hughes Research Laboratories and revolutionized many technologies [6]. In particular, high power pulsed lasers have been increasingly used to generate shock waves in materials, the pioneering experiments being those of Askaryon and Morez [7], White [8], Andelholm [9], and Inal and Murr [10].

Fairand and Clauer [11] and Clauer et al. [12] were the first to investigate the potential of laser shock strengthening for the hardening of aluminum alloys and stainless steel. Their intended application was welds and they demonstrated significant increase in fatigue life. By using three sequential shock events, they increased the hardness of stainless steel from KHN 250 to KHN 400. The fatigue life of welded aluminum alloys 5456 and 2024 was significantly increased and this was attributed to residual compressive stresses at the surface. They were able to generate pressures in the 6 to $10 \mathrm{GPa}$ range using a transparent (to the laser beam) overlay. This confined the vaporized metal at the surface and increased the peak pressure. The duration of the shock pulse produced in lasers is lower by orders of magnitude than the one in the explosively or gun accelerated projectiles: 100 ps-10 ns. Lasers can be used in different modes: direct laser irradiation of the surface; conversion to soft x-rays that then launch a compression or shock wave into a sample; generation of a "plasma piston" that then compresses or shocks a sample; 
or acceleration of a flyer foil [13]. Five experimental configurations are shown in Figure 2. The pressures achievable by lasers can be very high, in the range of hundreds of GPa.

Direct drive refers to direct laser bombardment upon the surface of a material. The target material will influence the process greatly, but in essence the laser interacts with the electrons near the surface, exciting them to elevated energy states if the laser energy is sufficient. As bombardment endures, the temperature rises tremendously. The result is a portion of the surface, either molten or plasma, expanding quickly away from the laser spot (Figure 3). As the matter travels away from the surface, the target is accelerated by the rocket effect: $\dot{x}=v \ln \left(m_{0} / m_{1}\right)$. The resultant shock velocity is determined by the amount of mass (m) and the velocity (v) at which the matter exhausts. If we assume that some fraction of the laser intensity is absorbed and that the resultant shock pressure is proportional to the density times the square of the velocity we arrive at the classical Lindl [14] equation: $P=C_{A}\left(I_{12} / \lambda_{\mu m}\right)^{2 / 3}$. The pressure (P) is given in GPa, the laser intensity $\left(\mathrm{I}_{12}\right)$ is given in $\mathrm{TW} / \mathrm{cm}^{2}$, and wavelength $(\lambda)$ is in micrometers. The adsorption coefficient $\left(\mathrm{C}_{\mathrm{A}}\right)$ is $\sim 40$ for low $\mathrm{Z}$ matter. An empirical value of the scaling power is 0.71 from diamond ablators at OMEGA (Section 2.3 ). The laser intensity can be found simply using dimensional analysis; $\mathrm{I}=\mathrm{E} /(\mathrm{At})$, where $\mathrm{E}$ is the laser energy, $\mathrm{t}$ is the pulse duration, and $\mathrm{A}$ is the spot size of the laser. An example high-energy laser pulse that might be generated at OMEGA is $532 \mathrm{~nm}, 50 \mathrm{~J}, 3 \mathrm{~ns}$, and a spot size of $1 \mathrm{~mm}^{2}$ resulting from a square phase plate - the resulting peak pressure is an astounding 100 GPa.

Mikkola and coworkers $[15,16]$ were some of the first to explore the ultra-short shock-compression regime. They used exploding foils to drive flyer plates with small 
thicknesses and generated pressure pulses with durations as low as $0.04 \mathrm{~ns}$. The $\mathrm{Cu}-8.7$ at. \% Ge alloys that they studied twinned profusely by virtue of the stacking fault energy that was significantly lower than that of copper. They observed an interesting phenomenon: the twin width increased with shock pulse duration from $0.8 \mathrm{~nm}$ at $0.017 \mathrm{~ns}$ to $5 \mathrm{~nm}$ for $0.3 \mathrm{~ns}$. These comparisons are made for a constant pressure of $20 \mathrm{GPa}$, although different pressures showed the same effects. This had an effect on the residual hardness that was unexpected. The nanosized twins enhanced the hardness more than their larger counterparts, and therefore a decrease in strengthening was observed with an increase in pulse duration beyond 0.04 ns. This work showed that ultrashort pulse durations produce unique substructural effects.

Figure 4 shows the essential differences between the flyer plate (Fig. 4a) and laser shock generated stress waves (Fig. 4b) [17]. In Fig. 4b, the laser pulse is triangular shaped, which leads to nonstationary propagation (i.e. the peak pressure decays continuously with distance from the free surface). The laser pulse can also be shaped and quasi-isentropic waves can be launched through proper design. This presents advantages and disadvantages.

One advantage is that the short duration and rapid decay of the wave generates a self-quenching regime by which the temperature equilibrates in time of around one millisecond or less. In flyer plate experiments this time is on the order of seconds. Thus, post shock recovery effects take place and the structure observed is very different form the one generated during the passage of the shock pulse. Another advantage is the pressures that can be reached in laser shock are higher than in flyer plate experiments. Pressures between 0 and $200 \mathrm{GPa}$ can easily be achieved by simply changing the laser 
energy or time scale. However, the pressure front obtained in early laser experiments was not easily controlled in the laser shock method; pressure spikes were commonly observed. The Gaussian shape of the laser beam can create 'hot spots' and the use of diffraction techniques through the phase plate can minimize this. This problem can also be minimized by the use of flyer foils or ablators generating pressure plasmas, which homogenize the pressure over the front. Figure 5 shows the decay of the pressure pulse as calculated by the code HYADES [18]. It can be seen that the short duration of the shock pulse created by the 3-ns laser pulse leads to a rapid pressure decay in the specimen. Snapshots of the pressure profiles in the $\mathrm{Cu}$ at times from 1 to $200 \mathrm{~ns}$ and up to a depth of $1 \mathrm{~mm}$ are shown in Figures 5(a), (b), and (c) for three initial energy levels: 70, 200, and $300 \mathrm{~J}$. The amplitude of the pressure wave in the sample decays substantially and the pulse duration broadens as a function of distance. In Figure 5(d) one can see the decrease of the maximum pressure at three energy levels. This decay is exponential. This is a result of the release at the front free surface immediately following the end of the laser pulse, generating a triangular-shaped pulse. In the other hand, geometries using a flyer plate decay much more gradually after a plateau in which the pressure is constant. In addition to the quenching of the specimen that is much faster than for larger duration shots, with a much greater chance of preserving the shock structure for post shock characterization, this decay in pressure with distance presents another significant advantage: one single specimen can provide a broad range of shock conditions and transmission electron microscopy can reveal features of an entire range of conditions, if the distance from the surface is measured and correlated to the pressure pulse calculated by HYADES. 
A disadvantage is that the area that can be shocked is much reduced to $1-2 \mathrm{~mm}^{2}$, rendering characterization and mechanical testing only accessible in a transmission electron microscope (TEM), scanning electron microscope (SEM), and micro or nanoindentation. Another disadvantage is that the planarity of shock front is not as exact as in flyer plate experiments and the front develops curvature that is followed by the formation of a crater. However, the use of a phase plate significantly reduces the lack of planarity.

This article will review our work on laser shock loading, initiated in the early 2000s, with an emphasis on recent results. This review supplements and updates the extensive article published by our group in Dislocations in Solids, 2009 [5]. We compare the results of experiments, analyses and molecular dynamics modeling in order to present a unified, comprehensive viewpoint of the effects of high amplitude stress waves generated by lasers on fcc and bcc metals. A brief discussion is introduced on dislocation dynamics (DD) modeling, which extends the spatial capability of molecular dynamics, restricted to $100 \mathrm{~nm}$ size boxes. We briefly discuss shock-induced phase transformations and amorphization in silicon. We also review our results on spalling or tensile failure generated by the reflection of shock waves at free surfaces and recent results on pressure/shear amorphization in silicon.

\section{Principal Pulsed Laser Facilities in the United States: NIF, JLF and LLE}

Worldwide there are many state-of-the-art laser shock facilities. The Laboratoire pour l'Utilisation des Lasers Intenses (LULI) facility in France and the Central Laser Facility (Vulcan) in the UK, Shanghai Institute of Optics and Fine Mechanics (SIOM) in China are examples. However, given budget constraints, proposal acceptance 
probabilities and travel fund limits, the laser facilities chosen for specific experiments are based more on practical reasons than in finding the perfect fit. Solely within the United States there are great options for researchers to use laser facilities for their experimental work: the National Ignition Facility (NIF) at the Lawrence Livermore National Laboratory (LLNL) is currently transforming from a purely government-only to outside user facility for a fraction of its operations. The Jupiter Laser Facility (JLF) at LLNL, including Janus and Titan, is currently open to academic institutions. The Omega laser facility, part of the Laboratory for Laser Energetics (LLE) at the University of Rochester, NY is accessible to international and academic users. They are described below.

\subsection{National Ignition Facility}

The National Ignition Facility (NIF) at LLNL in Livermore, California is the largest and highest-energy research laser facility in the world. Spanning three football field lengths, Fig. 6a, NIF has 40,000 optics that guide, reflect and amplify 192 laser beams to a single point with the capability of reaching $1.85 \mathrm{MJ}$ of energy during a span of only 10-20 nanoseconds. This translates to pressures and temperatures such as those in the interiors of stars and giant planets. With such an incredible capability, NIF is paving the way to research advancing national security, inertial fusion energy, and disciplines including astrophysics, materials science, planetary science, particle physics and nuclear science.

NIF is operational twenty-four hours a day, five days a week, and firing one or more laser shots a day. Recent use of NIF has widened to include academic institutions as well through the NIF Discovery Science program [19,20]. This transition is slowly taking shape within the NIF user community. 


\subsection{Jupiter Laser Facility}

The Jupiter Laser Facility (JLF) is part of the Physical and Life Sciences Directorate at LLNL and consists of 5 laser facilities: Titan, Janus, Callisto, Europa and COMET. The mission of JLF is to both carry out frontier science and also to promote collaborations with university institutions, engage young scientists with aspirations of becoming NIF users, and support inter-laboratory research in High Energy Density (HED) science.

Janus has been operational since the 1980s and is a several hundred joule-class Nd-glass laser system, Figure 6b. Janus has an east and west beam, each capable of producing 1 to 20 nanosecond pulses with energies ranging from $20 \mathrm{~J}$ to $1 \mathrm{~kJ}$ with a wavelength of $1053 \mathrm{~nm}$ (infrared) or $528 \mathrm{~nm}$ (green), as of 2014 [21].

\subsection{Omega Laser Facility}

OMEGA is a high-energy laser facility at the Laboratory for Laser Energetics (LLE) at the University of Rochester, New York. In 1970 LLE was founded by Dr. Moshe Lubin; together with Kodak they built Delta, a 4 beam laser system. More and more laser beams were added as the years progressed and by 1995 the laser system had increased to a total of 60 laser beams now known as OMEGA, Figure 6c. The entire length is $\sim 100 \mathrm{~m}$ and the chamber has a diameter or $\sim 8 \mathrm{~m}$. In 2008 the OMEGA extended performance (EP) system was opened, fashioned similar to NIF; this four-laser system gave OMEGA the capability of firing short pulsed shots [22].

\subsection{Coherent Light Sources}

Beyond the large pulsed laser facilities (e. g., Janus and Omega) the transformative capability of the coherent light sources such as Stanford's Linear 
Accelerator LCLS (Linac Coherent Light Source) and the Argonne APS (Advanced Photon Source) provide a new tool which can be a qualitative jump in our ability to capture extreme events. This geometry is poorly suited for recovery but can be used successfully in connection with X ray diffraction. Milathianaki et al. [26] subjected copper to pulses with a diffraction of $\sim 300$ ps with a spot of $30 \times 30 \mu \mathrm{m}^{2}$. Independent $X$ rays probed the structure, through diffraction, as the sample was loaded. At the strain rate of $10^{9} \mathrm{~s}^{-1}$ imparted by this system, the elastic limit for copper was estimated to be 73 $\mathrm{GPa}$, corresponding to a shear stress of $\sim 37 \mathrm{GPa}$. This is significantly higher than the theoretical shear strength calculated by Mackenzie's method, as described by Kelly, in "Strong Solids": 12 GPa [27]. This difference remains unexplained.

\section{Compression of Materials in Laser Target Chambers}

Many specimen configurations and geometries can be subjected to laser compression and release, depending on the desired effect. The simplest (and commonly used) setup is just a disk or rectangle in the center of which the laser beam is directed. It the specimen is sufficiently thin, spalling can be generated and the variation in thickness actually provides the flexibility of different stress pulses. In the case of phase plate, one can illuminate a region of $1 \mathrm{~mm}^{2}$. If reflected waves are a concern, the assembly developed in explosive and gas gun experiments can be miniaturized and applied to specimens, generally of cylindrical geometry. Lateral momentum trapping devices, such

as a hollow cylinder that is press fit (by heating and then allowing it to cool after insertion of specimen) are also useful to trap reflectd lateral waves. A momentum trap of the same material is also used to prevent reflected tensions. The use of the laser shock has a significant advantage over thicker flyer plates in that the wave attenuates right away. 
Figure 5 shows that, a a distance of $1 \mathrm{~mm}$ from the front surface the maximum amplitude is approximately $10-20 \%$ of the initial value. In our experiments, we have often used specimens with a $3 \mathrm{~mm}$ diameter and equal thickness. The reflections are minimized in this case. However, for brittle materials such as ceramics, Si, and Ge, the encapsulation of specimen in a material with similar shock impedance (approximated as the product of density and sound velocity) . is essential for successful recovery.

The samples are placed in the center of the laser chamber and the laser beams are focused to converge on the surface of the specimen, placed at the extremity of the recovery tube [23]. Figure 7 shows an example of a recovery tube illuminated by multiple laser beams. Perfect synchronization of the laser pulses is required to produce the desired stress pulse. Figure 8 shows a longitudinal section of a recovery tube in which the target sample is placed [24]. For convenience in the preparation of transmission electron microscopy (TEM) specimens, the diameter of the samples is often chosen to be $3 \mathrm{~mm}$. The specimen may be protected by a momentum trap and lateral tube to trap as much as possible the reflected tensile pulses. The momentum trap and lateral tube should be of close impedance (sound velocity $\mathrm{x}$ density) to minimize reflection. The rapid attenuation and reflection of stress pulses in the laser-induced shocks can cause recovery problems in brittle specimens due to failure by low amplitude tensile pulses. The configuration used in Fig. 9 is not one of direct laser shock on the surface. Upon shock breakout from the ablator, the expansion of the releasing plasma across the vacuum gap produces a ramp compression wave into the specimen that steepens into a shock. Some degree of pulse control can be obtained by proper design of the lasers. Thus, a quasi-isentropic loading, in which the strain rate is decreased from $10^{9}$ to $10^{6} \mathrm{~s}^{-1}$, can be achieved. Nevertheless, 
the quasi-isentropic ramp-compression pulse only travels a very short distance into the specimen before it steepens into a shock at around 50-60 ns.

Another characteristic feature of laser compression is the formation of a crater. An example of a laser shock induced crater is shown in tantalum (Fig. 10) shocked to a peak pressure of $110 \mathrm{GPa}$ [24]. Associated with the formation of the crater are voids and bubbles close to the surface due to melting and vaporization (Fig. 11) [23,25]. Hence one should be aware of the unique advantages and limitations of laser shock compression. Although the surface region and the adjoining layer to a depth of $50 \mu \mathrm{m}$ can be made to ramp up isentropically, the bubbles, which are evidence of vaporization serve as a warning that the temperatures may be high over the regions close to the surface.

\section{Science Questions Addressed by Laser Shock}

Laser compression and release enables the exploration of extreme regimes not achievable before in a controlled reproducible manner with diagnostics, computations and physics based analysis. Laser compression can produce strain rates up to $10^{9} \mathrm{~s}^{-1}$ with duration of $\sim 1$ to several tens of ns. This method of deformation is well suited for direct comparison with molecular dynamics in which the strain rates imposed are on the order of $10^{8}-10^{10} \mathrm{~s}^{-1}$ with durations of up to $100 \mathrm{ps}$. These two techniques have been used by our group with great success. This combined methodology has been applied to investigate five fundamental materials science concepts:

1) Finding the maximum strength of materials under extreme strain rates and pressures in compression

2) Exploring homogenous generation versus dislocation multiplication in shock compression

3) Defining the slip/twinning transition in materials 
4) Studying the defects and kinetics of void nucleation and growth in this extreme regime of high strain rate tension

5) Investigating shock and shear-induced transformations and amorphization.

These will be discussed in Sections 4.1-4.5.

\subsection{Strength Under Extreme Compression through in situ X-ray Diffraction}

In 2003, Meyers et al. [28] conducted strength experiments using laser compression on single crystalline [100] copper. The samples were mounted into recovery tubes and laser shocked at varying energies to study the material's response at increasing pressures. Within the laser target chamber an X-ray diagnostic was set up for in-situ Laue (transmission) and Bragg (reflection) diffraction. Figure 12 shows the experimental setup inside the laser chamber. The laser beam hits the target (shown as a thick line) from the top and the x-ray source (creating Bragg and Laue diffraction) impacts the sample from the bottom. As mentioned previously, an advantage to the laser shock method over the flyer plate method is that it offers the means to incorporate such diagnostics into the experimental process [28,29]. Figure 13 shows how the distortions from laser compression are viewed at the atomic scale from Laue and Bragg diffraction. In the experimental setup, the incoming X-ray beam was incident on the back surface of the sample, opposite to the laser beam. the shock was moving in the [100] direction The (200) atomic planes were diffracted in Bragg geometry, and the (020) planes that were perpendicular to the shock velocity were imaged in Laue geometry [28]. The distortions of the planes at the shock front give valuable information as to the deformation generated. Figure 13a shows the ambient lattice (unshocked). For example, Figure 13b shows purely elastic compression through the lattice, where the Laue diffraction angle 
would remain unaltered and the Bragg diffraction angle would detect the shock compression of the (200) lattice planes. In purely elastic loading, the relationship between the maximum shear stress, $\tau_{\max }$, and the hydrostatic component of stress $(\mathrm{P})$ is

$$
\tau_{\max }=\frac{3(1-2 v) P}{2(1+2 v)}
$$

where $v$ is the Poisson's ratio and pressure $\mathrm{P}$ is the hydrostatic component of the stress system imposed by shock loading. At a critical applied pressure, the deviatoric component of the stress is sufficient to generate defects (dislocations, twinning, phase transitions). The purely elastic distortion of the lattice generates uniaxial strains ( $\left.\varepsilon_{22}=\varepsilon_{233}=0, \varepsilon_{11} \neq 0\right)[30]$.

If the material has zero strength, ${ }_{11}={ }_{22}={ }_{33}$ and the state of stress relaxes to hydrostatic. However, material strength creates a partial relaxation of the strains. This was used to calculate the elastic shear stresses in the lattice. This method was applied to the X-ray diffraction results, in transmission (Bragg diffraction) and reflection (Laue diffraction). Both transversal and longitudinal strains could be approximately measured through the change of $\mathrm{d}$ spacing. Figure 13c shows elastic and plastic compression where the Laue and Bragg diffraction angles detect dislocations behind or at the shock front that do not accommodate for the distortions in the lattice. Diffraction calculations of the Laue and Bragg angles thus give insight into the distortions at the shock front. Lastly, Figure 13d shows hydrostatic compression where the Laue and Bragg diffraction angular changes are equal to each other (the perpendicular and parallel compressions are equal). This corresponds to a zero shear-strength case [28,31]. 
Figure 14 shows time integrated and time resolved results for copper at a calculated shock strength (pressure) of $18 \mathrm{GPa}$. This is related to the following ratio,

$$
\left(\frac{d}{d_{0}}\right)^{3}=\frac{V}{V_{0}}
$$

where $d=0.966 d_{0}$ is the compressed lattice spacing, and the ratio $V / V_{0}=0.903$ is the ratio of final to initial specific volumes. The results presented in Figure 14 suggest that the copper lattice has elastic strains similar to the example illustrated in Figure 13c, where the dislocations do not accommodate entirely the distortions (deviatoric stresses) in the lattice. Taking purely elastic considerations, the deviatoric stresses can be calculated, taking $\varepsilon_{11}$ as the strain in the [100] direction of the lattice (parallel to the direction of the shock velocity) and $\varepsilon_{22}=\varepsilon_{33}$ as the strain in the [010] direction perpendicular to the shock velocity. Thus the shear strain is expressed as

$$
\gamma=\frac{\pi}{2}-\tan ^{-1}\left(\frac{1+\varepsilon_{11}}{1+\varepsilon_{22}}\right)
$$

In a single crystal, the stresses are related to the strains by the $\mathrm{C}_{\mathrm{ij}}$ elastic constant matrix. For crystals with cubic symmetry, there are three: $\mathrm{C}_{11}, \mathrm{C}_{12}$, and $\mathrm{C}_{44}$. For the simpler, isotropic, case, the shear stress, $\tau$, is related to the strain by:

$$
\tau=2 \mathrm{G} \gamma
$$

which is effectively Hooke's Law. This is the shear stress that the lattice can sustain elastically, or the shear strength. The strain rate at the shock front is estimated as the strain divided by the rise time of the shock pulse,

$$
\dot{\varepsilon}=\frac{\varepsilon}{t_{f}}=\frac{\frac{2}{3} \ln \left(\frac{V}{V_{0}}\right)}{t_{f}}
$$


where $V_{0}$ is the initial specific volume and $V$ is the specific volume of the material after lattice compression. The shear strength (from Eq. 4) and strain rate for copper for an 18 GPa shock are plotted in Figure 15, together with measurements at lower strain rates by Tong et al. [32]. This estimate is surprisingly consistent with other measurements by split Hopkinson bar and pressure shear experiments.

This technique was extended to higher shock strengths by Murphy et al. [33] who measured the shear strain and shear stress in copper at pressures exceeding $100 \mathrm{GPa}$, Fig. 16. The strength they observed for $\mathrm{P}_{\text {shk }} \sim 100 \mathrm{GPa}$ was measured to be $1.2 \mathrm{GPa}$ at a strain rate of $10^{10} \mathrm{~s}^{-1}$. The time resolved diffraction was measured from two different reflection planes and crystalline anisotropy was incorporated into the calculations: $G$ was replaced by $\mathrm{C}_{44}$. There is a significant effect of orientation on the elastic stiffness and shear modulus.

\subsection{Phase Transitions Under Extreme Compression through in situ X-ray Diffraction}

In situ diffraction experiments are also used to determine phase transformations and the classic example is iron, undergoing an $\alpha(\mathrm{bcc})$ to $\varepsilon(\mathrm{hcp})$ displacive transformation at a pressure of $13 \mathrm{GPa}$; this was investigated by Kalantar et al. [34]. The experimental setup uses a film plate at a specified angle with the specimen. The diffraction cones intersect the plane along diffraction curves which can be calculated exactly and are shown in Fig. 17(a). The X-rays are created by laser illumination of a metal, generating k-shell X-rays that are emitted from the hot plasma and cross an aperture before hitting the sample. It is this 'backlighter' beam that is diffracted. Figure 17(b) shows the diffracted lines from iron prior to and during shock compression. The lines prior to compression are marked by squares; during compression, a state of uniaxial strain is 
established in the [001] oriented specimen, and the changing d spacing shift the lines in predictable ways that can be calculated. These are the triangular lines. However, the transformation to the hexagonal phase generates new lines which are marked with stars in Fig. 17(b). This experiment shows conclusively the formation of the new phase and its crystallographic relationship to the parent.

\subsection{Strength under Extreme pressures through Instability Evolution}

Another manner in which strength of metals can be inferred at the extreme pressures and strain rates imparted by laser shock is through the growth of instabilities. The extremely high strengths of materials deformed at high rates and $100 \mathrm{GPa}$ pressures are able to suppress the development of the RT instability significantly. A laser-based, experimental platform developed to study solid-state material dynamics at ultrahigh strain rates and pressures [1,35-37] is shown schematically in Fig. 18. Two laser beams are used: one to generate the pressures (in a quasi-isentropic mode, with a much reduced temperature rise) and a second, through a backlighter, to capture the evolution of the ripples. The strain rates are very high, on the order of $107 \mathrm{~s}^{-1}$, corresponding to a characteristic strain of $\sim 10 \%$ over a characteristic time scale of $\sim 10 \mathrm{~ns}$. The ramped plasma drive accelerates this rippled foil, and the interface (between the stagnating plasma drive and the rippled foil) is subject to the Rayleigh Taylor instability. Figure 18 shows the variation in the size of the ripples as a function of time for tantalum subjected to a pressure of approximately $100 \mathrm{GPa}$. In order to measure dynamic plasticity and infer flow stress under high-pressure, high-strain-rate conditions, the growth of these ripples through a process of Rayleigh-Taylor (RT) instability, is measured and compared with predictions from different constitutive models. The growth would be zero if the strength 
is infinite and, as indicated in Fig. 18c, maximum for a fluid with zero strength. Specimens with different grain sizes, varying from 0.25 to $92 \mathrm{um}$, were tested and the growth rate is independent of grain size. Different constitutive equations were used to estimate the evolution of the ripples and are shown in plot: Steinberg-Guinan (SG); Preston-Tonks-Wallace (PTW) [38]; Steinberg-Lund (SL) [39]; and a multiscale model named Livermore Multi Scale (LMS) [40]. The strength of the tantalum was dialed in to

determine the best fit and two factors were determined: (a) the strength of 6 GPa using LMS gives the best fit; (b) there seems to be no effect of grain size, at the strain rate investigated $\left(10^{7} \mathrm{~s}^{-1}\right)$.

\subsection{Dislocation Generation Models at the Shock Front}

Plasticity from laser shock loading differs from that of the Hopkinson bar where the deformation state in the lattice is one of uniaxial stress; it is also assumed to be homogeneous over the length of the specimen. In shock compression, the uniaxial strain state is reached with three principal non-zero stress components [30]. There are two distinct mechanisms that describe dislocation generation, motion and interactions in a metal: homogeneous dislocation generation and dislocation multiplication. Studying these two mechanisms is important because the dislocation density in the metal directly affects its mechanical behavior.

\subsubsection{Homogeneous Dislocation Generation}

Meyers [30] in 1978 predicted dislocation densities based on a homogeneous nucleation model and compared their analysis with results from different investigations. Dating back to 1958 , Smith was the first to publish work on dislocation mechanisms 
induced by an explosively driven shock [4,41]. Shortly after, Hornbogen [42] added to Smith's work on edge dislocations by considering screw and edge components forming loops. The concept of homogeneous dislocation nucleation by Meyers et al. followed. Consecutive layers of dislocation loops are nucleated and remain behind the shock front, relaxing deviatoric stresses, moving either towards or away from the front at different velocities. He proposed that dislocations are homogenously generated at the shock front and move by loop expansion to accommodate the deviatoric stresses in the lattice [30]. Since the dislocations cannot keep up with the front, successive loop events take place generating the residual dislocation density. This mechanism was later improved and its predictions were critically compared with computational and experimental (transmission electron microscopy) results.

Two cases are considered: moving and stationary dislocations. For stationary dislocations, the following equation illustrates the spacing between dislocation arrays:

$$
h_{1}=\frac{0.8(1-v)}{\pi^{2} b} d_{2}^{2}
$$

$h_{1}$ is the spacing between dislocation generation events (perpendicular to front) and $d_{2}$ is the spacing along the front. In Fig. 19, only $\mathrm{h}$ is used, and it represents both $h_{1}$ and $h_{2}$. As the dislocations try to move as fast as the shear wave velocity, driven by the residual shear stress, the spacing between sequential dislocation loop generation events is $h_{2}$ (Figure 19). The dislocations try to "catch up" to the shock front, resulting in an increase from $h_{1}$ to $h_{2}$,

$$
h_{2}=h_{1}\left[1+\frac{k V_{d p}}{U_{s}}\right]
$$


where $V_{d p}$ is the dislocation velocity, $k$ is an orientation factor and $U_{s}$ is the shock wave velocity. Assuming the shock velocity is approximately equal to the sound speed velocity,

$$
U_{s} \cong C_{0}=\left(\frac{\lambda+2 \mu}{\rho}\right)^{\frac{1}{2}}
$$

When the dislocation velocity is zero, $h_{2}$ reduces to $h_{1}$. When the dislocation velocity equals the shear wave velocity, $V_{s w}, h_{2}$ reaches a maximum. Assuming dislocation density is primarily based on the two conditions of $h$, static (where $V_{d p}=0$ ) and dynamic (where $V_{d p}=V_{s w}$ ) dislocations, a model was fit to experimental results from three separate sources.

The dislocation density, $\rho$, can be analytically expressed in terms of the specific volume, $V$, for a static dislocation,

$$
\rho=\frac{2 \pi^{2}}{0.8(1-v) k^{3} b_{0}^{2}}\left(\frac{V}{V_{0}}\right)^{-2 / 3}\left[1-\left(\frac{V}{V_{0}}\right)^{1 / 3}\right]^{3}
$$

where $v$ is Poisson's ratio, $k$ is an orientation parameter, $b_{0}$ is the Burgers vector, $V_{0}$ is the initial specific volume, and $V$ is the specific volume of the material after lattice compression $[28,43]$.

The analytical dislocation density expression can also be expressed as a function of pressure, $P$, obtained through the Rankine-Hugoniot equations and the equation of state, 


$$
P=\frac{C_{0}^{2}\left(1-\frac{V}{V_{0}}\right)}{V_{0}\left[1-S\left(1-\frac{V}{V_{0}}\right)\right]^{2}}
$$

where $C_{0}$ is the sound velocity and $S$ is a parameter from the equation of state that is specific to the material [28]. Rearranging Eq. (10) gives,

$$
\frac{V}{V_{0}}=1-\frac{1}{2 P V_{0} S^{2}}\left[2 P S V_{0}+C_{0}^{2} \pm \sqrt{\left(2 P S V_{0}+C_{0}^{2}\right)^{2}-4 P^{2} V_{0}^{2} S^{2}}\right]
$$

Substituting Eq. (11) into Eq. (9) lends to the dislocation density in terms of pressure $[28,44]$. Figure 20 shows experimental results by a number of investigators and predictions from the homogeneous dislocation generation mechanism. The differences are of at least one order of magnitude and neither the early model [30] nor improvements [28]were able to predict values of dislocations densities that approached experimental results. This remained a mystery until 2008.

Transmission electron microscopy (TEM) images of laser shock compressed copper, Fig. 21a showed large and small dislocation loops. When compared to the dislocation loop densities in undeformed copper samples, laser compressed copper has much higher loop densities. This firmly suggested that dislocation loop generation was an important event during elastic to plastic transition in laser compression. Consistent with results found in copper and other fcc metals, partial dislocation loops were emitted in MD simulations during laser shock compression in copper and Figure 21b shows a crosssectional view from MD simulations of the staking faults in single crystal [001] Ni after compression just above the HEL. The three-dimensional view, Fig. 21c, from the MD 
simulations shows the plastic zone lagging behind the partial dislocation loops generated from the leading elastic precursor wave. Figures 21a-c illustrate the qualitative match between observations, the analytical homogeneous dislocation model, and simulations.

The homogeneous dislocation density model proposed and improved by Meyers for copper was extended to nickel in 2008 [44]. The nucleation of partial dislocation loops is schematically shown in Figure 22a at slip planes behind the shock front. Figure $22 \mathrm{~b}$ shows that all four stacking-fault variants were activated during shock loading under TEM observation [28,45]. In 2008 Jarmakani et al. [44] compared the laser shock experimental results for a range of pressures from 10-80 $\mathrm{GPa}$ of nickel to MD simulations. The comparison of the experimental, analytical, and computational components of this work clarified a long-standing disagreement in the literature: it is now recognized that a large fraction of the dislocations must be annihilated upon unloading. Thus, MD predictions, analytical calculations and experimental findings are in agreement if this annihilation is incorporated.

Analytical models were also applied to predict the critical pressures for the cellto-stacking-fault transition and the onset of twinning as a function of grain-size and stacking-fault energy. In 2009 Meyers et al. [5] recalculated the densities but the values are still very different from experimental results.

Molecular dynamics (MD) simulations of stress unloading in nickel were conducted by Jarmakani et al. [44] and compared to experiments. Dislocations generated during experimental compression were annihilated upon unloading, an effect often neglected in MD simulations up until this point in time. Figure 23 shows the pressure 
increase and decrease during compression and release, respectively. The stacking faults are shown, since only defective atoms from the MD simulations [44] and imaged. The stacking-fault density clearly decreases significantly after unloading. Interestingly, the dislocation densities in the simulations after unloading matched the experimental results by Murr [46] fairly well. In Fig. 24 it can be seen that there is a disparity between theory (analytical results) and experimental results. Holian and Lomdahl [47] were responsible for the important molecular dynamics simulations in 1998 showing the shock waveinduced plasticity dependence on shock strength for a fcc lattice. In Figure 24, the two dimensionless parameters are defined by Holian and Lomdahl [47] have the following meaning :

- $\quad$ Plasticity is defined as the ratio $a_{0} l l$, where $a_{0}$ is the lattice parameter and $l$ is the dislocation spacing. The higher the dislocation density, the smaller is the 'plasticity.'

- $\quad$ Shock strength. This is defined as $u_{p} / c_{0}$, the ratio between particle velocity and sound speed. The larger the pressure, the higher $u_{p}$.

Experimental results by Murr [46] and computational results for $\mathrm{Cu}$ from Cao et al. [45] are also included in this plot as well as theoretical and molecular dynamics results for nickel.. The molecular dynamics results for nickel and the experimental results of Murr fall beneath the trends of the theoretical (homogeneous dislocation generation model) and molecular dynamics results of Cao et al. [45]. These results show that the shock generates a high dislocation density, that decreases upon unloading due to dislocation annihilation.

Focus transitioned from fcc to bcc metals, especially with the introduction of lasers as a new technique to induce shocks. Lasers have the advantages of generating 
higher pressures, albeit at much reduced durations. Flyer plate experiments are better at generating highly controlled and prescribed planar waves with well-established configurations. However, the pulse durations are much higher. Lu et al. [24] studied the recovery of single crystalline tantalum subjected to laser shock compression. Figure 25a$\mathrm{b}$ shows a schematic of a shock front propagating through a bec crystal, moving through the single crystal in the [001] orientation. Fig. 25a shows the \{110\} slip planes of a [001] bcc single crystal. Four of the six slip planes in $\{110\}$ have equal Schmid factors, which are shown as sides to the pyramid. The dislocation loops that nucleate on these planes are shown schematically and their Burgers vectors are <111>, parallel to the slip plane directions. Fig. $25 \mathrm{~b}$ shows the $\{112\}$ planes with the Burgers vectors also with the same direction and magnitude. The comparison of Figs. 22a and 25a illustrates the differences between the fcc and bcc structures. In bcc metals, the MD simulations (in monocrystals) do not predict the homogeneous nucleation of dislocation loops up to pressures on the order of $60 \mathrm{GPa}$ (for tantalum). In the bcc structure, the PN is very high, and dislocations are only generated by molecular dynamics if flaws, such as voids (or vacancy clusters), existing dislocations, or grain boundaries, are introduced into the crystal. Thus, other mechanisms have to be postulated, which are explored in Section 3.4.2.

\subsubsection{Dislocation Multiplication}

Face-centered cubic and body-centered cubic metals have very different strainrate and temperature response by virtue of the significant differences in the activation volume for plastic deformation and magnitude of Peierls-Nabarro barriers. This has a direct bearing on the nucleation of dislocation loops, absent in MD simulations for tantalum at $\mathrm{P}<60 \mathrm{GPa}$. 
The Orowan [48] expression, which connects continuum and dislocation mechanics, has a simple form:

$$
{ }_{p}=k b l
$$

where ${ }_{p}$ is the plastic shear strain, $k$ is the Taylor factor, $\rho$ is the mobile dislocation density, $b$ is the Burgers vector, and $l$ is the mean distance that the dislocation moves. The increase in dislocation density by multiplication is the alternative to the homogeneous dislocation nucleation at the shock front. Every crystal has preexisting, pre-shock dislocations and these act as multiplication sources. Equation 12 is barely expressed mathematically in the original paper by Orowan and this has given rise to the idea that Orowan never proposed the Orowan equation. However, he got very close to it. Taking the time derivative considering both $\rho$ and $l$ to be strain-rate dependent:

$$
\dot{\gamma}_{p}=\frac{d \gamma_{p}}{d t}=k b\left(\rho \frac{\partial l}{\partial t}+l \frac{\partial \rho}{\partial t}\right)=k b(\rho v+l \dot{\rho})
$$

where $v$ is the mobile dislocation velocity, equal to $\partial l / \partial t$. In order to apply Eqn. 13 to the shock regime, an analytical dislocation velocity expression was obtained by fitting the MD simulation data from Tang et al. [49] and Deo et al. [50]:

$$
v=v_{s}\left\{1-A \exp \left[-B\left(\frac{\sigma}{\tau_{p}}\right)^{m}\right]\right\}
$$

where the predicted maximum velocity is equal to the shear wave velocity, $v_{s}, A, B$ and $m$ are parameters and $\tau_{\mathrm{p}}$ is the Peierls-Nabarro stress. 
The strain rates in shock compression are extremely high. The relationship between the strain rate at the shock front and the pressure observed experimentally, and expressed mathematically by Swegle and Grady [51], is:

$$
P \propto \dot{\varepsilon}^{1 / 4}
$$

Experimental work done on tantalum by Furnish et al. [52] yielded the following relationship between pressure and strain rate,

$$
\dot{\varepsilon}=27.34 \times 10^{-36} \times P_{\text {shock }}^{4}
$$

The relationship between uniaxial and shear strain, necessary to connect Eqns. 13 and 16, is established below for a state of uniaxial strain, imposed by the laser shock compression experiments:

$$
\begin{aligned}
& \tau=\frac{\sigma_{1}-\sigma_{2}}{2}=\frac{1-2 v}{2(1-v)} \sigma \\
& G \gamma=\frac{1-2 v}{2(1-v)} \varepsilon E \frac{(1-v)}{(1+v)(1-2 v)}=\frac{\varepsilon E}{2(1+v)}=G \varepsilon
\end{aligned}
$$

where $\tau$ is the shear stress, $\sigma$ is the normal stress, $\gamma$ is the shear strain, $\varepsilon$ is the normal strain, $v$ is the Poisson ratio, $G$ is the shear modulus, and $E$ is the Young's modulus.

Equation 18 simplifies to $\gamma=\varepsilon$, and thus $\dot{\gamma}=\dot{\varepsilon}$ follows. The evolution of dislocation density is a function of the strain rate, since both the velocity and $\dot{\rho}$ are dependent on it. The velocity as a function of applied stress has been first expressed by the Johnson-Gilman [53] equation and then by more realistic expressions predicting a maximum velocity equal to the shear-wave speed, as shown in Fig. 26 and Eqn. 14. The change in dislocation density, $\dot{\rho}$, has been expressed by Kocks [54] as the difference between the rate of generation and the rate of annihilation of dislocations: 


$$
\dot{\rho}=\dot{\rho}_{g e n}-\dot{\rho}_{a n n}
$$

The following expression was proposed by Barton et al. [55](a similar expression was proposed earlier by Kocks [54]):

$$
\dot{\rho}=\left(k_{1} \rho^{1 / 2}-k_{2} \rho\right) \dot{\gamma}
$$

An expression connecting the dislocation density to the strain rate can be developed.

$$
\rho=\rho\left(\dot{\varepsilon}_{p}, v_{d}\right)=\rho(P)=\frac{\dot{\varepsilon}_{p}-M b v_{d} t R \dot{\varepsilon}_{p}}{\left(M b v_{d}-\frac{M b v_{d} t R \dot{\varepsilon}_{p}}{\rho_{s a t}\left(\dot{\varepsilon}_{p}\right)}\right)}
$$

By applying the Swegle-Grady equation (Eqns. 15 and 16), one can predict the curves shown in Figure 27. It shows dislocation density calculations as a function of pressure from separate laser shock experiments using both the homogeneous dislocation generation model, and the Orowan multiplication model. The analytically calculated dislocation density values for the homogeneous dislocation generation are magnitudes higher than the experimentally measured values. As mentioned earlier in Section 3.2.1, this is due to fact that the analytical model does not incorporate two important factors that take place during shock compression: relaxation during shock compression and defect motion/annihilation during rarefaction. By not taking these two factors into consideration it can be understood why the analytical model predicts a higher dislocation density. It is clear that dislocation multiplication compares much more favorably with experimental results. The data are bracketed by the Kocks [54] and the Barton et al. [55] models. More details on the analytical expressions can be found in Lu et al. [24]. 
As a conclusion, the MD does not predict dislocation generation up to pressures

of $60 \mathrm{GPa}$. This is the threshold for homogeneous dislocation generation and exceeds the $\mathrm{G} / 2 \pi$ theoretical shear strength prediction.

\subsection{Slip-Twinning Transition}

Slip and twinning are competing defects in the deformation process in fcc and bcc metals. Meyers et al. [56] and Murr et al. [57] extensively studied the mechanics of twinning in fcc metals and alloys. Both twinning and slip are dominated by the shear stresses acting in the direction of displacement and either on the slip or twin plane. The slip-twinning transition can be approximated to the first order as the shear stress for both being equal,

$$
\tau_{s}=\tau_{t}
$$

The shear stresses can be related to the perpendicular $\left(\sigma_{22}\right)$ and parallel $\left(\sigma_{11}\right)$ components of the shock propagation during elastic loading (uniaxial strain state), given by Eqn. 17.

Meyers et al. [28] and Jarmakani et al. [44] extended the initial efforts to find the constitutive prediction of the slip-twinning transition in shock compression of copper and nickel. The stress required for slip to take place is determined, in a simple but elegant manner, by the Zerilli-Armstrong (Z-A) relation for bec metals in the thermal activation regime,

$$
\sigma_{s}=\sigma_{s}^{*}+C_{2} e^{-C_{3} T} \dot{\varepsilon}^{C_{4} T}+k_{s} d^{-1 / 2},
$$

where $C_{2}, C_{3}, C_{4}$ and $k_{s}$ are Z-A parameters specific to the material. The constitutive relation to twinning in bcc metals is developed as a function of grain size, temperature, 
strain and strain rate. The stress required for twinning to take place is determined by the Armstrong and Worthington [58] equation,

$$
\sigma_{T}=\sigma_{0}+m\left(\frac{G(P, T) b}{C_{1}}\right)^{1 / 2}\left[\frac{U^{*}}{R T} \ln \frac{\dot{\varepsilon}}{\dot{\varepsilon}_{0}}\right]^{1 / q} d^{-1 / 2}
$$

where $G(P, T)$ is an expression of shear module as function of pressure and temperature. The strain rate is related to the pressure, as stated before, by the Swegle-Grady equation (Eqn. 14).

In 2012, Lu et al. [24] found experimental evidence of twinning in tantalum after laser shock under TEM observation, Fig. 28. After further investigation, a slip-twinning transition was found for tantalum at a critical threshold range shown in Figure 29 between 29-35 GPa. This range is due to the temperature rise in tantalum during loading [24]. Recovered samples from the experiments yielded dislocation density results calculated from TEM observation. The twinning density is plotted in Fig. 30, and compared to experimental results for tantalum dislocation density by Gray and Vecchio [59] and Hsiung [60]. After the onset of twinning, the dislocation density no longer increases significantly because the shear strain is accommodated by both slip and twinning. This was already observed by Mikkola and coworkers [15,16]. Figure 30 shows the drop in dislocation density after the onset of twinning. In Figure 26 the same decrease in the rate of dislocation generation can also be seen. This can be analytically expressed by:

$\gamma=\left\{\begin{array}{ccc}M b \rho_{\perp} l & \text { if } & \dot{\gamma}<\dot{\gamma}_{\text {threshold }} \\ M b \rho_{\perp} l+f_{T} \gamma_{T} & & \dot{\gamma}>\dot{\gamma}_{\text {threshold }}\end{array}\right.$ 
where $\rho_{\perp}$ is the dislocation density, $f_{T}$ is the twin fraction, $\gamma_{T}$ is the twin strain $(=0.707$

in BCC), and $\dot{\gamma}_{\text {threshold }}$ is the threshold strain rate at which the slip-twinning transition occurs.

\subsection{Dynamic Failure by Spalling: Void and Crack Nucleation and Growth}

Spall, fracture, cracking, and failure in materials are of interest for a wide range of fields applications including ballistic penetration, dynamic fragmentation in hypervelocity particle target interactions, optical, diagnostic equipment hazards in high energy density (HED) facility chambers, and asteroid and meteor dynamics as they enter the atmosphere and break up [61-63]. Previous research by the U.S. Army Research Laboratory (ARL) has studied spall in several different metals, in order to provide protection from 'behind-armor debris'. There has been extensive study of spall strength and its mechanisms of dynamic failure in fcc metals. The following sections will briefly introduce spall, the numerical derivation of spall strength and the important experiments that have led to the research achieved today (e.g. Meyers and Aimone [64]).

When a shock wave travels through a specimen and reaches the free rear surface of the sample, a rarefaction (or release) wave is formed and travels back into the sample. When the pressure source on the driven (front) side of the sample turns off, a release wave travels into the sample from the front side, as well. When these two release waves meet, a strong tensile stress is created, which can lead to failure (spall). If the tensile pulse exceeds a critical threshold stress, the material undergoes failure, known as spall. Spall is classified into ductile and brittle. Meyers and Aimone [64] present a cornucopia of examples of both. Spall due to brittle failure can occur through trans- or inter-granular cleavage. Spall caused by ductile failure in the material can also occur through trans- and 
inter-granular void nucleation, growth, and coalescence, necking or shear localization. The mechanism of spall failure can easily be identified by scanning electron microscopy. Brittle fracture is clearly identified by sharp cracks on the surface of the sample whereas ductile failure is easily identified by its dimple like structures or shear markings.

Figure 31 shows the spall strengths of different materials ranked by their bulk moduli; and compares them with their cohesive strengths, calculated by Grady [65]. Two observations can be made from the plot:

1) The cohesive (and spall) strengths increase with bulk modulus. Grady's [65] expression has the form

$$
\sigma_{t h}=\sqrt{\frac{U_{c o h} B_{0}}{8 V_{0}}},
$$

where $\sigma_{t h}$ is the theoretical spall strength, $U_{c o h}$ is the specific cohesive energy, $B_{0}$ is the bulk modulus, and $V_{0}$ is the specific volume at zero pressure for the material.

2) The spall strengths are proportional to the cohesive strengths; the spall strength in gas-gun experiments $(\sim 1 \mu$ s duration) is lower than the one in laser experiments (1-10 ns, Fig. 31). Although experimental techniques have developed to further push the limits of metals, spall strength results still fall short of their theoretical values [65]. In 2000, Moshe et al. [66] did get very close to experimentally reaching the theoretical spall strength in $\mathrm{Cu}$ and $\mathrm{Al}$. The values in Fig. 31 for lasers are collected from various sources: Eliezer et al. [67] for Al, Jarmakani et al. [68] for V, Schneider et al. [18] for Cu. At very short time scales (20 and $100 \mathrm{ps}$ laser durations) and extreme strain rates (above $10^{8} \mathrm{~s}^{-1}$ ), the spall strength for $\mathrm{Al}$ was just below the equation of state (theoretical strength) and the spall strength for $\mathrm{Cu}$ reached its theoretical limit within the experimental error bars [66]. 
In 2010 Jarmakani et al. [68] studied the spall strength of vanadium, a bec metal. Single and polycrystalline vanadium was subjected to laser shock compression. Spalling in the polycrystals occurred by a ductile tearing mechanism that favored grain boundaries. In the single crystals it occurred by a mixture of cleavage fracture along the $\{010\}$ planes and ductile dimple fracture (laser-induced). Spall strengths in vanadium were found to be considerably higher than results from gas gun experiments, and the monocrystals showing a higher value than polycrystals (Fig. 31). This higher spall strength is suggestive of a strong strain rate dependence, consistent with the nucleation, growth, and coalescence kinetics of voids or microcraks and the strain rate sensitivity embedded in the Curran-Shockey-Seaman theory [69]. The laser experimental data by Jarmakani et al. [68] for spall strength of vanadium agrees favorably with gas-gun results when the strain rates are on the order of $\sim 10^{6} \mathrm{~s}^{-1}$. When the strain rate increases in the laser shock experiments to $\sim 10^{7} \mathrm{~s}^{-1}$, the spall strength of vanadium also increases, falling on the laser experiment trend (Fig. 31).

The experimental and computationally obtained spall strengths of tantalum are plotted vs. strain rate in Figure 32 [70-80] adapted from Hahn et al. [81]; this plot shows in an eloquent manner how spall strength increases with strain rate. This a clear consequence of the time and stress dependence of the processes of void/crack nucleation, growth, and coalescence. The extreme strain rate of $10^{13} \mathrm{~s}^{-1}$ provides the maximum spall strength that corresponds to the cohesive strength of tantalum. Indeed, the results are consistent and the ultimate tensile strength at this strain rate is $\sim 35 \mathrm{GPa}[81]$.

Dating back to 1986, Christy et al. [82] performed recovery experiments on polycrystalline copper plates studying void nucleation during spall. It was initially 
assumed that spall strength had a direct correlation to hardness, however, that was proven during the experiments to be untrue. Instead it was found that spall strength was correlated to nucleation, growth and coalescence of voids.

From the micrograph in Figure 33, a peanut shaped void can be seen. This TEM was obtained using the Kratos microscope operating at $1 \mathrm{MeV}$; this enabled penetration into thicker specimens so that the entire void can be visualized. This and similar voids in copper were observed to primarily nucleate and grow along grain boundaries. Thus it was concluded that grain boundaries are favored initiation sites for voids during spall [82] . Profuse plastic deformation is also seen through the dark region surrounding the void. Additional examples of voids nucleating at grain boundaries are seen in Fig. 34 [64]. The slip lines emanating from the voids can be seen.

Copper containing inclusions had a much reduced spall strength because of the availability of nucleation sites. Christy et al. [82] compared the spall strength of small and large grained polycrystalline copper samples. For large-grained specimens the majority of voids initiated at grain boundaries. The rationale for these results is presented in Figure 31. Additionally, monocrystalline copper exhibits the highest spall strength, in contrast to the quasistatic behavior, in which monocrystals have the lowest yield strength. The differences are rationalized by the schematic plot of Figure 35 . The yield strengths of monocrystalline, coarse grain sized, and fine grain sized obey the classic Hall-Petch relationship. The interfacial strength of grain boundaries, dictated by the imperfect matching of atoms, impurities, and even second phase particles, is assumed to have a lower strain rate sensitivity than the plastic deformation processes by dislocations within the material. Thus, the failure mechanism changes to grain-boundary separation at a 
critical strain rate. Experimental results by Kanel [83] and Kanel et al. [84] for mono and polycrystalline copper, aluminum, and iron were the first to establish the generality of this 'inverse' response.

The significant effect that microstructural effects have on the spalling strength and morphology can be evaluated from the single plot summarizing the results obtained by Minich et al. [85] for monocrystalline ([100], [110], [111]) and polycrystalline copper (grain sizes of 8, 50, and $133 \mathrm{um}$ ). This is shown in Figure 36. Although these results were obtained in gas-gun experiments at pulse durations of $\sim 1$ us, using flyer plate thickness of $1.5 \mathrm{~mm}$, they show the same trends observed under laser shock: monocrystals have higher spall strength than polycrystasls. Copper with $\mathrm{SiO}_{2}$ particles was also studied and shows the predicted significant drop in spall strength.

Molecular dynamics simulations provide an additional tool for understanding the mechanisms of void nucleation, growth and coalescence. The work done by Traiviratana and Bringa [86] in the late 2000's has opened up incredible insight into the inner workings of spall at the atomic level. The effects of the loading orientation with respect to the crystals was studied to investigate the potential outcome of dislocation emission. It was postulated by Lubarda et al. [87] that loop emission occurred at $45^{\circ}$ angles from the slip plane. Figure 37 shows a schematic of the [110] loading direction (z-axis) with two slip planes drawn as dashed lines intersecting the surface of a dislocation loop at $45^{\circ}$ angles. These represent maximum shear stress planes. Two types of loops were postulated: prismatic loops (already well known in the literature) and a new type of shear loop. Similar to the dislocation distribution predicted by Lubarda et al. [87] . 
In 2010, molecular dynamics simulations of copper by Bringa et al. [86] showed the growth sequence of dislocations for the [100], [110], and [111] loading directions. For [111] loading, the three slip planes are shown in Figure 38 where the leading and trailing partials and stacking faults are schematically illustrated. The emission of shear loops is seen in a most eloquent manner. This occurs first for the leading partial dislocation and then for the trailing dislocation and the stacking fault shown in MD.

For bcc crystals the configurations of loops are quite different. Generation and propagation of loops were studied by Tang et al. [49] in hydrostatic tension. Figure 39 shows the three shear loops on the three slip planes. These shear loops transform into prismatic loops, as they grow. A similar mechanism was observed by Remington et al. [88] under a nanoindenter. Figure 40 shows a sequence of shear loop formation in tantalum under uniaxial compression at high strain rates $\left(\sim 10^{8} \mathrm{~s}^{-1}\right)$ from molecular dynamics simulations [49]. In this case, the shear loops do not transform into prismatic loops. It is thought that this is due to the difference in stress state.

Illustrating the void growth process, Tang et al. [49] also calculated analytically the dislocation emission from a single void in uniaxial tension for fcc and bcc metals. Figure 41 shows a shear loop emission from the top view of a void. As the void undergoes uniaxial tension, volume must be conserved such that dislocations are emitted at the exterior of the void surface. Figure $41 \mathrm{~b}$ shows this process in the side view, as the shear loop is being emitted, the radius of the void increases, leading to void growth [89].

The stress required for loop emission decreases with increasing size of the void. This was both computationally observed and analytically predicted. Using an analysis 
based on Rice-Thomson [90] and Weertman [91], Tang et al. [89] developed the equations below for fcc and bcc metals, respectively:

$$
\begin{aligned}
\frac{\sigma_{\max }}{G_{112}} & =\frac{0.165 \tau_{\max }}{G_{112}}=0.165\left[\frac{2 \gamma}{G_{112} \pi \rho b_{p}}+\frac{b_{p}\left(2-v_{112}\right)}{4 \pi\left(1-v_{112}\right) R_{1}} \ln \frac{8 m R_{1}}{e^{2} \rho b_{p}}+\frac{2 \gamma_{S F}}{G_{112} b_{p}}\right] \\
\frac{\sigma_{\max }}{G_{111}} & =\frac{0.224 \tau_{\max }}{G_{111}}=0.224\left[\frac{2 \gamma}{G_{111} \pi \rho b}+\frac{b\left(2-v_{111}\right)}{4 \pi\left(1-v_{111}\right) R_{1}} \ln \frac{8 m R_{1}}{e^{2} \rho b}\right]
\end{aligned}
$$

where $G_{112}$ is the shear modulus of the $\{111\}$ planes shearing in the <112> direction, $\mathrm{G}_{<111>}$ is the shear modulus of the $\{110\}$ planes in the $\left\langle 111>\right.$ direction, $b_{p}$ is Burgers vector of partial dislocations, $\rho$ is the core radius factor, $v$ is the Poisson ratio, $R_{l}$ is the dislocation loop radius (assumed to be equal to one half the void radius $\mathrm{R}$ ), $m$ is a constant, $\gamma$ is the surface energy and $\gamma_{S F}$ is the stacking fault energy [89]. Figure 42 shows the predictions. Unfortunately, MD simulations are limited to void sizes less than $20 \mathrm{~nm}$. Nevertheless, the agreement between computations and analysis is excellent.

Tang et al. [89] furthermore show in a molecular dynamics simulation a sequence of events beginning with a tri-vacancy within tantalum, Figure 43a, undergoing uniaxial tension at a high strain rate of $10^{9} \mathrm{~s}^{-1}$ emitting planar defects (stacking faults) at $11 \%$ strain, Figure 43b [89]. This shows that defects as small as a trivacancy act as nucleation sites for spalling.

Another interesting aspect of void growth is that there is anisotropy of plastic deformation leading to non-spherical shapes. In 1983 Meyers and Aimone [64] also observed void growth due to spall in a nickel sample. Nickel is a very ductile metal, giving the sample a dimpled appearance on the spalled surface after laser compression and release. A few, however, important observations were made by Meyers et al. about 
the void initiation sites. Many voids were analyzed to have initiated homogeneously. Two of these are shown, in Fig. 44a and b. Prior to this, in 1972, Stevens, Davison and Warren [92] performed spall experiments on monocrystalline aluminum disks. Portions of the spalled samples were cut out and the damage was analyzed through optical and scanning electron microscopy, Figure 44c. The voids had octahedral form with $\{111\}$ planes as faces. A kinematic model based on dislocation motion was proposed to describe the growth of the voids. The dynamic model describing the rate of growth for an individual void was combined with an empirically established nucleation model in order to get the total growth rate in a spall sample [92]. However, the interpretation given by Stevens et al. [92], that bulk dislocations slipped into the voids, being annihilated, was wrong. The explanation for the faceting of voids lies in the emission of dislocation loops from the void. This is shown in Figure 44d: the sequence shows how the shear loops on $\{111\}$ planes generate the faceting of a void. These MD simulations of monocrystalline copper show the growth of voids with stacking fault emission when undergoing uniaxial tension [86].

In 2013 Tang et al. [93] once again studied the uniaxial tension on polycrystalline tantalum, a model bcc metal. Figure 45a shows nanocrystalline Ta with grain size of 27.3 $\mathrm{nm}$ undergoing tension, shown by the left and right arrows. Figure $45 \mathrm{~b}$ shows crack nucleation preferentially at the grain boundary and the propagation of a traveling crack from this initiation cite, Figure 45c. Figures 45 b-c are mostly devoid of dislocations, however, in Figure 45d twinning can be observed. A schematic of the principal mechanisms of failure in nanocrystalline bcc metals is shown in Figure 46. The nucleation, growth and coalescence of cracks at the grain boundaries are depicted in 
Figure 46a-b, and the crack initiation at the grain boundary and subsequent twinning in the crystalline is shown schematically in Figure 46c-d [93] .

It should be mentioned that these MD simulations are conducted on 'annealed' tantalum devoid of dislocations. When shock compression of nanocrystalline Ta precedes spalling, profuse dislocation and twin generation occurs, and the boundaries are the principal sources. Upon tension produced by spalling, void initiation and growth takes place at the grain boundaries, but in a much more ductile manner, propitiated by the existing defects. This is shown in the sequence shown in Figure 47. Only the grain boundaries are imaged by the MD simulation and the separation and formation of the voids is seen. Dislocations and twins are also present.

In polycrystalline fcc metals, $\mathrm{MD}$ simulations of nanocrystalline $\mathrm{Cu}$ also predict void formation at grain boundaries and accompanied by profuse dislocations emission. This is shown in the sequence displayed in Figure 48, which shows a slice of copper being deformed in tension. The light blue lines designate stacking faults, which are bound by a leading and trailing partial dislocation [86].

\subsection{Dislocation Dynamics vs. Molecular Dynamics}

Dislocation dynamics (DD) has evolved with the increase in capacity of computers and the early work by Kubin and Canova [94,95] is noteworthy, although the power of this methodology was rather limited in the early days. The beginnings of this approach date from the 1980s. In DD the dislocations are modeled as lines embedded in a elastic matrix and the laws of dislocation mechanics are applied to each line, that is divided into segments. Thus, the crystallography of slip, cross slip, dislocation loops, reactions, bowing of dislocation lines, dislocation d dynamics, long and short range 
interactions of dislocations, and other dislocation characteristics are incorporated into the model. The application to shock compression of copper was implemented by Zbib and Diaz de la Rubia [96] and Shehadeh et al. [97-100]. Shehadeh et al. [97-100] were able to follow the evolution of dislocation density behind the front. Figure 49 shows three snapshots at different times: 67,90 , and 120 ps. The nucleation of loops at the front is seen: at 67 ps (a), a large number of loops are generated, which can be seen to grow at 90 ns. At $120 \mathrm{ps,} \mathrm{new} \mathrm{arrays} \mathrm{of} \mathrm{loops} \mathrm{are} \mathrm{again} \mathrm{homogeneously} \mathrm{nucleated.} \mathrm{A} \mathrm{criterion} \mathrm{for} \mathrm{the}$ nucleation of these loops was established using MD results: the shear stress required was $3 \mathrm{GPa}$. Additionally, a minimum longitudinal stress $\sigma_{11}=30 \mathrm{GPa}$ was established as a threshold. As the loops expand they eventually interact and an extremely high dislocation density is generated. The uniaxial strain state is relaxed to a hydrostatic stress as the loops are generated and propagate. This is shown in Fig. 49(d). Initially $\sigma_{11}$ is higher than $\sigma_{22}=$ $\sigma_{33}$. The three stresses become equal as do the strains through the nucleation and motion of dislocations. As this occurs, the dislocation density increases and reaches a saturation. The dislocation density evolution was measured and is plotted in Fig. 49 (e) as a function of pressure. It is compared with MD predictions and is lower. It was also found that the density decreases with the increase in the rise time of the wave. In Figure 49 the rise time is $50 \mathrm{ps}$. This is consistent with residual hardness measurements made by Jarmakani et al. [68], who observed enhanced hardening in shock as compared to isentropic loading.

\subsection{Shock and Shear-Induced Phase Transitions and Amorphization}

Thadhani and Meyers [101], Chang and Meyers [102], and Sano et al. [103] used reflected waves to induce martensitic transformations in Fe-Ni-C and Fe-Ni-Mn alloys. 
There is a dilatational strain of $\sim 0.05$ in these alloys as well as a shear strain and in the spall area; both contribute to increase the martensite start $\left(\mathrm{M}_{\mathrm{s}}\right)$ temperature. These experiments, using pulse durations of $100 \mathrm{~ns}$ to $3 \mu \mathrm{s}$, enabled the analysis of the martensitic transformation in an extreme regime not achievable by other means. The experiments provided valuable information on the kinetics of martensitic nucleation and propagation.

The high pressures induced by shocks lead to new phases, such as the hexagonal $\varepsilon$ phase in iron and a high pressure stishovite phase in silica. The $\alpha$ to $\varepsilon$ transformation induced by laser compression was studied by Kalantar et al. [104] among others. In tantalum, Lu et al. [105] confirmed the earlier discovery of an $\omega$ phase by Hsiung and Lassila [106,107]. The earlier experiments were conducted using an explosivelyaccelerated flyer plate and the threshold pressure was $\sim 40 \mathrm{GPa}$. In laser experiments, the critical pressure for the transformation increased to $\sim 65-70 \mathrm{GPa}$. Figure 50 shows an experimentally obtained transmission electron micrograph of the $\omega$ phase after shock compression of Ta crystals with three orientations: a) [110], b) [111], and c) [123]. The dark field TEM shows the illuminated $\omega$ phase which has boundaries that tend to be faceted. In the dark field image, a diffraction spot characteristic of the $\omega$ phase was used. MD simulations revealed nanosized nodules of hexagonal phase, seen in Fig. 50 d. It should be mentioned that in order for these nodules to be generated, pressure as well as shear had to be applied. The shear stresses generated by the uniaxial strain state imparted by shock compression are essential. 
We show below that the shear component of stress cannot be neglected. The uniaxial shock stress in the direction of shock wave propagation, $\sigma_{11}$, hydrostatic pressure, $\mathrm{P}$, and maximum shear stress, $\tau_{\max }$ are related by,

$$
\sigma_{11}=\mathrm{P}+\frac{4}{3} \tau_{\max }
$$

In elastic compression, the ratio of $\tau_{\max }$ over $\mathrm{P}$, for cubic materials loaded along [001] direction, is given as a function of the elastic constants:

$$
\frac{\tau_{\max }}{P}=\frac{3\left(C_{11}-C_{12}\right)}{2\left(C_{11}+2 C_{12}\right)}
$$

where $\mathrm{C}_{11}$ and $\mathrm{C}_{12}$ are second order elastic moduli (pressure dependent stiffness), resulting in a pressure dependent relationship between shear stress and hydrostatic pressure. MD simulations using the Modified Tersoff potential (MOD) potential [108] show good agreement with the pressure dependent stiffness and predicted $\frac{\tau_{\max }}{P}$ as a function of pressure. For Ta, this ratio is 0.49 , using zero pressures values. This is below the HEL. When the material flows plastically, the shear stress relaxes.

Zhao et al. [109] reported that a laser driven shock, at a strain rate of $\sim 10^{7} \mathrm{~s}^{-1}$, induced amorphization in monocrystalline silicon. A bulk amorphous surface layer and amorphous bands along favorable crystallographic directions were observed, both under TEM in recovered samples and in MD simulations. It was proposed that large shear stress coupled with high pressure leads to the amorphization. In this investigation, Zhao et al. [110] addressed this phenomenon, analyzing it quantitatively using thermodynamic parameters. Additionally, they evaluated the crystallization process from the amorphous state. Assuming a purely elastic compression, the shear stress, which was $\sim 0.5 \mathrm{P}$ at zero 
stress, was reduced to $\sim 0.3 \mathrm{P}$ at $14 \mathrm{GPa}$. Thus, it reached significant values. Figure 51a,b shows both pressure-induced amorphization and shear-induced amorphization. The laser irradiation creates pressure at the surface that launches a shock pulse traveling from left to right. The shear-induced amorphization forms in bands that either oriented at $45^{0}$ to the shock front (maximum shear stress) or in specific crystallographic orientations at which dislocations propagate ((111) planes). The MD simulations are in full agreement with experimental results, as can be seen in Figs. $51 \mathrm{c}, \mathrm{d}$. One can see the amorphization at a lower (c) and higher (d) energy and the predictions are consistent with TEM observations.

\section{Conclusions}

This review presents the fundamental aspects of laser shock compression and release and addresses five fundamental materials science questions in the extreme regime of pressure and strain rate:

- It was possible to experimentally determine the strength of metals (copper) under extreme strain rates and pressures. An abundance of work has been done successfully determining the strength up to strain rates of $10^{10} \mathrm{~s}^{-1}$. This has been enabled by using X-ray diffraction in conjunction with laser compression. Different reductions of lattice parameters along planes parallel and perpendicular (or inclined) to the shock wave propagation direction enable extraction of the deviatoric elastic strains and associated shear stresses under these extreme strain rates. For copper, the strength reaches a level of $1.2 \mathrm{GPa}$ at $10^{10} \mathrm{~s}^{-1}$ for a shock strength of $\sim 100 \mathrm{GPa}$. A methodology that is complementary to the determination of lattice spacings during compression is the observation of the of ripples on the 
surface of metals subjected to compression; these ripples grow by a process of Raleigh-Taylor instability. The strength can be extracted at strain rates on the order of $10^{7} \mathrm{~s}^{-1}$ and pressures up to $100 \mathrm{GPa}$.

- Homogenous dislocation generation was proposed by Meyers [30] in 1978 as a defect generation mechanism in fcc metals. Through MD simulation, it is shown that in fcc metals, homogeneous dislocation generation takes place, whereas in bcc metals dislocation multiplication is favored, except for the strongest shocks.

- The slip-twinning transition in metals was explored as it relates to pressure and shear induced stresses. A simple criterion establishing a critical shear stress for the initiation of twinning provides satisfactory results that compare well with experimental observations.

- Void and crack nucleation, growth and coalescence is discussed and the results of laser experiments are compared with gas-gun spall strength. It is shown that the time plays an important role in determining the spall strength. Thus, spall strengths in laser shock are higher than in explosively or gas gun experiments.

- The analysis of the time dependent spall strength by molecular dynamics enables the prediction of the ultimate tensile strength of metals, when the strain rate is on the order of the Debye frequency.

- A mechanism for void initiation and growth based on dislocation loop emission from the void surface is proposed and validated for both bcc and fcc structures by MD simulations.

- Shock and shear-induced structural changes observed experimentally and reproduced with $\mathrm{MD}$ simulations are presented. In tantalum, a $\beta$-to- $\omega$ 
transformation is observed for pressures above $65 \mathrm{GPa}$. Silicon undergoes amorphization above $12 \mathrm{GPa}$. Both the transformation and amorphization are pressure and shear induced.

\section{Acknowledgements}

This research was funded by the Department of Energy Stewardship Science grant (DE-NA0002080) under the principal investigator Marc A. Meyers. Supplemental funding sources: National Laser Users Facility Grant (PE-FG52-09NA-29043) and UC Research Laboratories Grant (09-LR-06-118456-MEYM). We thank the staff of the Jupiter Laser Facility and Laboratory for Laser Energetics for support over the past fifteen years. A special gratitude goes to the current and past members of our group, Dr. B. Kad, Dr. H. Jarmakani, Dr. T. Traiviratana, Dr. C.H. Lu, Dr. Y. Tang, Dr. C. Ruestes, Dr. Tramontina, S. Zhao and to the LLNL researchers Dr. B. Maddox, Dr. H.S. Park, and Dr. C. Wehrenberg who were instrumental in the planning and execution of the laser shock experiments. We thank B. Wang for help with figures, S. Zhao for editing of the text.

\section{References}

[1] B.A. Remington, R.E. Rudd, J.S. Wark, From microjoules to megajoules and kilobars to gigabars: Probing matter at extreme states of deformation, Phys. Plasmas. 22 (2015).

[2] B. Hopkinson, A Method of Measuring the Pressure Produced in the Detonation of High Explosives or by the Impact of Bullets, Philos. Trans. R. Soc. Lond. Ser. Contain. Pap. Math. Phys. Character. 213 (1914) 437-456.

[3] F.R. Tuler, B.M. Butcher, A criterion for the time dependence of dynamic fracture, Int. J. Fract. Mech. 4 (1968) 431-437. doi:10.1007/BF00186808.

[4] C.S. Smith, L. Guttman, Measurement of internal boundaries in threedimensional structures by random sectioning, Trans AIME. 197 (1953) 81-87. 
[5] M.A. Meyers, H. Jarmakani, E.M. Bringa, B.A. Remington, Chapter 89 Dislocations in Shock Compression and Release, in: L. Kubin, J.P. Hirth (Eds.), Dislocations Solids, Elsevier, 2009: pp. 91-197. http://www.sciencedirect.com/science/article/pii/S1572485909015022.

[6] T.H. Maiman, Stimulated optical radiation in ruby, Pergamon Press LTD, Oxford, 1960.

[7] G. Askaryon, E. Morez, Use of Laser Generated Shocks to Improve Metals \& Alloys, JETP LETT. 16 (1963) 1638.

[8] R.M. White, Generation of elastic waves by transient surface heating, J. Appl. Phys. 34 (1963) 3559-3567.

[9] N. Anderholm, Laser-generated stress waves, Appl. Phys. Lett. 16 (1970) 113115.

[10] O. Inal, L. Murr, Laser-shock-induced microstructural changes and a comparison with explosive-shock-induced phenomena in metals: Field-ion and electron microscopic studies, J. Appl. Phys. 49 (1978) 2427-2434.

[11] B.P. Fairand, A.H. Clauer, Laser generation of high-amplitude stress waves in materials, J. Appl. Phys. 50 (1979) 1497-1502. doi:10.1063/1.326137.

[12] A.H. Clauer, J.H. Holbrook, B.P. Fairand, Effects of Laser Induced Shock Waves on Metals, in: M.A. Meyers, L.E. Murr (Eds.), Shock Waves High-Strain-Rate Phenom. Met., Springer US, 1981: pp. 675-702. http://link.springer.com/chapter/10.1007/978-1-4613-3219-0_38.

[13] S. Luo, D. Swift, T. Tierney IV, D. Paisley, G. Kyrala, R. Johnson, A. Hauer, O. Tschauner, P.D. Asimow, Laser-induced shock waves in condensed matter: some techniques and applications, High Press. Res. 24 (2004) 409-422.

[14] J.D. Lindl, P. Amendt, R.L. Berger, S.G. Glendinning, S.H. Glenzer, S.W. Haan, R.L. Kauffman, O.L. Landen, L.J. Suter, The physics basis for ignition using indirectdrive targets on the National Ignition Facility, Phys. Plasmas 1994-Present. 11 (2004) 339-491. doi:10.1063/1.1578638.

[15] S. LaRouche, D. Mikkola, Shock hardening behavior of Cu-8.7 Ge at very short pulse durations, Scr. Metall. 12 (1978) 543-547.

[16] R.N. Wright, D.E. Mikkola, S. LaRouche, Short Duration Shock Pulses as a Tool to Study the Time Dependence of Plastic Deformation, in: M.A. Meyers, L.E. Murr (Eds.), Shock Waves High-Strain-Rate Phenom. Met., Springer US, 1981: pp. 703-716. http://link.springer.com/chapter/10.1007/978-1-4613-3219$0 \_39$.

[17] B.Y. Cao, D.H. Lassila, M.S. Schneider, B.K. Kad, C.X. Huang, Y.B. Xu, D.H. Kalantar, B.A. Remington, M.A. Meyers, Effect of shock compression method on the defect substructure in monocrystalline copper, Mater. Sci. Eng. A. 409 (2005) 270-281.

[18] M. Schneider, B. Kad, M. Meyers, F. Gregori, D. Kalantar, B. Remington, Laserinduced shock compression of copper: orientation and pressure decay effects, Metall. Mater. Trans. A. 35 (2004) 2633-2646.

[19] E. Moses, R. Boyd, B. Remington, C. Keane, R. Al-Ayat, The National Ignition Facility: Ushering in a new age for high energy density science, Phys. Plasmas. 16 (2009). 
[20] B.A. Remington, Experiments in ICF, materials science, and astrophysics, EPJ Web Conf. 59 (2013) 1013. doi:10.1051/epjconf/20135901013.

[21] J.S. Wark, R.R. Whitlock, A.A. Hauer, J.E. Swain, P.J. Solone, Subnanosecond xray diffraction from laser-shocked crystals, Phys. Rev. B. 40 (1989) 5705.

[22] A. Heller, Collaboration Ignites Laser Advances, 1999. https://str.llnl.gov/str/Verdon.html.

[23] B.R. Maddox, H.-S. Park, C.-H. Lu, B.A. Remington, S. Prisbrey, B. Kad, R. Luo, M.A. Meyers, Isentropic/shock compression and recovery methodology for materials using high-amplitude laser pulses, Mater. Sci. Eng. A. 578 (2013) 354-361. doi:10.1016/j.msea.2013.04.050.

[24] C.H. Lu, B.A. Remington, B.R. Maddox, B. Kad, H.S. Park, S.T. Prisbrey, M.A. Meyers, Laser compression of monocrystalline tantalum, Acta Mater. 60 (2012) 6601-6620. doi:10.1016/j.actamat.2012.08.026.

[25] J.M. McNaney, M.J. Edwards, R. Becker, K.T. Lorenz, B.A. Remington, Highpressure, laser-driven deformation of an aluminum alloy, Metall. Mater. Trans. A. 35 (2004) 2625-2631. doi:10.1007/s11661-004-0208-3.

[26] D. Milathianaki, S. Boutet, G.J. Williams, A. Higginbotham, D. Ratner, A.E. Gleason, M. Messerschmidt, M.M. Seibert, D.C. Swift, P. Hering, J. Robinson, W.E. White, J.S. Wark, Femtosecond Visualization of Lattice Dynamics in Shock-Compressed Matter, Science. 342 (2013) 220-223. doi:10.1126/science.1239566.

[27] A. Kelly, N.H. Macmillan, Strong Solids, Oxf. Univ. Press Oxf. UK. (1986).

[28] M.A. Meyers, F. Gregori, B.K. Kad, M.S. Schneider, D.H. Kalantar, B.A. Remington, G. Ravichandran, T. Boehly, J.S. Wark, Laser-induced shock compression of monocrystalline copper: characterization and analysis, Acta Mater. 51 (2003) 1211-1228. doi:10.1016/S1359-6454(02)00420-2.

[29] A. Loveridge-Smith, A. Allen, J. Belak, T. Boehly, A. Hauer, B. Holian, D. Kalantar, G. Kyrala, R.W. Lee, P. Lomdahl, M.A. Meyers, D. Paisley, S. Pollaine, B. Remington, D.C. Swift, S. Weber, J.S. Wark, Anomalous Elastic Response of Silicon to Uniaxial Shock Compression on Nanosecond Time Scales, Phys. Rev. Lett. 86 (2001) 2349-2352. doi:10.1103/PhysRevLett.86.2349.

[30] M.A. Meyers, A mechanism for dislocation generation in shock-wave deformation, Scr. Metall. 12 (1978) 21-26. doi:10.1016/00369748(78)90219-3.

[31] E.M. Bringa, K. Rosolankova, R.E. Rudd, B.A. Remington, J.S. Wark, M. Duchaineau, D.H. Kalantar, J. Hawreliak, J. Belak, Shock deformation of facecentred-cubic metals on subnanosecond timescales, Nat. Mater. 5 (2006) 805809. doi:10.1038/nmat1735.

[32] W. Tong, R.J. Clifton, S. Huang, Pressure-shear impact investigation of strain rate history effects in oxygen-free high-conductivity copper, J. Mech. Phys. Solids. 40 (1992) 1251-1294.

[33] W.J. Murphy, A. Higginbotham, G. Kimminau, B. Barbrel, E.M. Bringa, J. Hawreliak, R. Kodama, M. Koenig, W. McBarron, M.A. Meyers, B. Nagler, N. Ozaki, N. Park, B. Remington, S. Rothman, S.M. Vinko, T. Whitcher, J.S. Wark, The strength of single crystal copper under uniaxial shock compression at 100 
GPa, J. Phys. Condens. Matter. 22 (2010) 65404. doi:10.1088/09538984/22/6/065404.

[34] D.H. Kalantar, G.W. Collins, J.D. Colvin, J.H. Eggert, J. Hawreliak, H.E. Lorenzana, M.A. Meyers, R.W. Minich, K. Rosolankova, M.S. Schneider, J.S. Stölken, J.S. Wark, In situ diffraction measurements of lattice response due to shock loading, including direct observation of the $\alpha-\varepsilon$ phase transition in iron, Int. J. Impact Eng. 33 (2006) 343-352. doi:10.1016/j.ijimpeng.2006.09.050.

[35] H.-S. Park, R.E. Rudd, R.M. Cavallo, N.R. Barton, A. Arsenlis, J.L. Belof, K.J.M. Blobaum, B.S. El-dasher, J.N. Florando, C.M. Huntington, B.R. Maddox, M.J. May, C. Plechaty, S.T. Prisbrey, B.A. Remington, R.J. Wallace, C.E. Wehrenberg, M.J. Wilson, A.J. Comley, E. Giraldez, A. Nikroo, M. Farrell, G. Randall, G.T. Gray III, Grain-Size-Independent Plastic Flow at Ultrahigh Pressures and Strain Rates, Phys. Rev. Lett. 114 (2015) 65502. doi:10.1103/PhysRevLett.114.065502.

[36] H.-S. Park, K.T. Lorenz, R.M. Cavallo, S.M. Pollaine, S.T. Prisbrey, R.E. Rudd, R.C. Becker, J.V. Bernier, B.A. Remington, Viscous Rayleigh-Taylor Instability Experiments at High Pressure and Strain Rate, Phys. Rev. Lett. 104 (2010) 135504. doi:10.1103/PhysRevLett.104.135504.

[37] H.-S. Park, B.A. Remington, R.C. Becker, J.V. Bernier, R.M. Cavallo, K.T. Lorenz, S.M. Pollaine, S.T. Prisbrey, R.E. Rudd, N.R. Barton, Strong stabilization of the Rayleigh-Taylor instability by material strength at megabar pressures, Phys. Plasmas. 17 (2010) 56314. doi:10.1063/1.3363170.

[38] D.L. Preston, D.L. Tonks, D.C. Wallace, Model of plastic deformation for extreme loading conditions, J. Appl. Phys. 93 (2003) 211-220. doi:10.1063/1.1524706.

[39] D.J. Steinberg, C.M. Lund, A constitutive model for strain rates from 10-4 to 106 s-1, J. Appl. Phys. 65 (1989) 1528-1533. doi:10.1063/1.342968.

[40] R.E. Rudd, A. Arsenlis, N.R. Barton, R.M. Cavallo, A.J. Comley, B.R. Maddox, J. Marian, H.-S. Park, S.T. Prisbrey, C.E. Wehrenberg, L. Zepeda-Ruiz, B.A. Remington, Multiscale strength (MS) models: their foundation, their successes, and their challenges, J. Phys. Conf. Ser. 500 (2014) 112055. doi:10.1088/1742-6596/500/11/112055.

[41] C.S. Smith, Metallographic Studies of Metals After Explosive Shock, Trans Met Soc AIME. Vol: 212 (1958) 574-589.

[42] E. Hornbogen, Shock-induced dislocations, Acta Metall. 10 (1962) 978-980.

[43] E.N. Hahn, S. Zhao, E.M. Bringa, M.A. Meyers, Supersonic Dislocation Bursts in Silicon, Sci. Rep. 6 (2016) 26977. doi:10.1038/srep26977.

[44] H.N. Jarmakani, E.M. Bringa, P. Erhart, B.A. Remington, Y.M. Wang, N.Q. Vo, M.A. Meyers, Molecular dynamics simulations of shock compression of nickel: From monocrystals to nanocrystals, Acta Mater. 56 (2008) 5584-5604. doi:10.1016/j.actamat.2008.07.052.

[45] B. Cao, E.M. Bringa, M.A. Meyers, Shock Compression of Monocrystalline Copper: Atomistic Simulations, Metall. Mater. Trans. A. 38 (2007) 2681-2688. doi:10.1007/s11661-007-9248-9.

[46] L.E. Murr, Residual Microstructure - Mechanical Property Relationships in Shock-Loaded Metals and Alloys, in: M.A. Meyers, L.E. Murr (Eds.), Shock 
Waves High-Strain-Rate Phenom. Met., Springer US, 1981: pp. 607-673. http://link.springer.com/chapter/10.1007/978-1-4613-3219-0_37.

[47] B.L. Holian, P.S. Lomdahl, Plasticity Induced by Shock Waves in Nonequilibrium Molecular-Dynamics Simulations, Science. 280 (1998) 20852088. doi:10.1126/science.280.5372.2085.

[48] E. Orowan, A type of plastic deformation new in metals, Nature. 149 (1942) 643-644.

[49] Y. Tang, E.M. Bringa, B.A. Remington, M.A. Meyers, Growth and collapse of nanovoids in tantalum monocrystals, Acta Mater. 59 (2011) 1354-1372. doi:10.1016/j.actamat.2010.11.001.

[50] C.S. Deo, D.J. Srolovitz, W. Cai, V.V. Bulatov, Stochastic simulation of dislocation glide in tantalum and Ta-based alloys, J. Mech. Phys. Solids. 53 (2005) 12231247. doi:10.1016/j.jmps.2005.01.003.

[51] J.W. Swegle, D.E. Grady, Shock viscosity and the prediction of shock wave rise times, J. Appl. Phys. 58 (1985) 692-701. doi:10.1063/1.336184.

[52] M.D. Furnish, L.C. Chhabildas, D.J. Steinberg, S.C. Schmidt, J.W. Shaner, G.A. Samara, M. Ross, Dynamical behavior of tantalum, AIP Conf. Proc. 309 (1994) 1099-1102. doi:10.1063/1.46296.

[53] W.G. Johnston, J.J. Gilman, Dislocation Velocities, Dislocation Densities, and Plastic Flow in Lithium Fluoride Crystals, J. Appl. Phys. 30 (1959) 129-144. doi:10.1063/1.1735121.

[54] U.F. Kocks, Laws for Work-Hardening and Low-Temperature Creep, J. Eng. Mater. Technol. 98 (1976) 76-85. doi:10.1115/1.3443340.

[55] N.R. Barton, J.V. Bernier, R. Becker, A. Arsenlis, R. Cavallo, J. Marian, M. Rhee, H.-S. Park, B.A. Remington, R.T. Olson, A multiscale strength model for extreme loading conditions, J. Appl. Phys. 109 (2011) 73501. doi:10.1063/1.3553718.

[56] M.A. Meyers, O. Vöhringer, V.A. Lubarda, The onset of twinning in metals: a constitutive description, Acta Mater. 49 (2001) 4025-4039. doi:10.1016/S1359-6454(01)00300-7.

[57] L.E. Murr, M.A. Meyers, C.-S. Niou, Y.J. Chen, S. Pappu, C. Kennedy, Shockinduced deformation twinning in tantalum, Acta Mater. 45 (1997) 157-175. doi:10.1016/S1359-6454(96)00145-0.

[58] R.W. Armstrong, P.J. Worthington, A Constitutive Relation for Deformation Twinning in Body Centered Cubic Metals, in: R.W. Rohde, B.M. Butcher, J.R. Holland, C.H. Karnes (Eds.), Metall. Eff. High Strain Rates, Springer US, 1973: pp. 401-414. http://link.springer.com/chapter/10.1007/978-1-4615-86968_22.

[59] G.T. Gray, K.S. Vecchio, Influence of peak pressure and temperature on the structure/property response of shock- loaded Ta and Ta-10W, Metall. Mater. Trans. A. 26 (1995) 2555-2563. doi:10.1007/BF02669413.

[60] L.L. Hsiung, Shock-induced phase transformation in tantalum, J. Phys. Condens. Matter. 22 (2010) 385702. doi:10.1088/0953-8984/22/38/385702.

[61] J.D. Hogan, J. Kimberley, K. Hazeli, J. Plescia, K.T. Ramesh, Dynamic behavior of an ordinary chondrite: The effects of microstructure on strength, failure and 
fragmentation, Icarus. 260 (2015) 308-319.

doi:10.1016/j.icarus.2015.07.027.

[62] J. Kimberley, K.T. Ramesh, The dynamic strength of an ordinary chondrite, Meteorit. Planet. Sci. 46 (2011) 1653-1669. doi:10.1111/j.1945-

5100.2011.01254.x.

[63] J. Kimberley, K. Ramesh, O. Barnouin, P. Swaminathan, C. Ernst, The Dynamic Fracture of Rocky Bodies: Applications to Planetary Impact Problems, Johns Hopkins APL Tech. Dig. 28 (2010) 242.

[64] M.A. Meyers, C.T. Aimone, Dynamic fracture (spalling) of metals, Prog. Mater. Sci. 28 (1983) 1-96. doi:10.1016/0079-6425(83)90003-8.

[65] D.E. Grady, The spall strength of condensed matter, J. Mech. Phys. Solids. 36 (1988) 353-384. doi:10.1016/0022-5096(88)90015-4.

[66] E. Moshe, S. Eliezer, Z. Henis, M. Werdiger, E. Dekel, Y. Horovitz, S. Maman, I.B. Goldberg, D. Eliezer, Experimental measurements of the strength of metals approaching the theoretical limit predicted by the equation of state, Appl. Phys. Lett. 76 (2000) 1555-1557. doi:10.1063/1.126094.

[67] S. Eliezer, I. Gilath, T. Bar-Noy, Laser-induced spall in metals: Experiment and simulation, J. Appl. Phys. 67 (1990) 715-724. doi:10.1063/1.345777.

[68] H. Jarmakani, B. Maddox, C.T. Wei, D. Kalantar, M.A. Meyers, Laser shockinduced spalling and fragmentation in vanadium, Acta Mater. 58 (2010) 4604-4628. doi:10.1016/j.actamat.2010.04.027.

[69] L. Seaman, D.R. Curran, D.A. Shockey, Computational models for ductile and brittle fracture, J. Appl. Phys. 47 (1976) 4814-4826. doi:10.1063/1.322523.

[70] B. Glam, M. Werdiger, Y. Horovitz, E. Moshe, S.L. Pistinner, Dynamic strength of tantalum under impact, J. Phys. Conf. Ser. 500 (2014) 112029. doi:10.1088/1742-6596/500/11/112029.

[71] J.-P. Cuq-Lelandais, M. Boustie, L. Soulard, L. Berthe, J. Bontaz-Carion, T. de Resseguier, Investigation of laser shock induced ductile damage at ultra-high strain rate by using large scale MD simulations, AIP Conf. Proc. 1426 (2012) 1167-1170. doi:10.1063/1.3686487.

[72] L. Soulard, J. Bontaz-Carion, J.P. Cuq-Lelandais, Experimental and numerical study of the tantalum single crystal spallation, Eur. Phys. J. B. 85 (2012) 1-15. doi:10.1140/epjb/e2012-30269-9.

[73] A.K. Zurek, W.R. Thissell, J.N. Johnson, D.L. Tonks, R. Hixson, Micromechanics of spall and damage in tantalum, J. Mater. Process. Technol. 60 (1996) 261267. doi:10.1016/0924-0136(96)02340-0.

[74] J.M. Rivas, A.K. Zurek, W.R. Thissell, D.L. Tonks, R.S. Hixson, Quantitative description of damage evolution in ductile fracture of tantalum, Metall. Mater. Trans. A. 31 (2000) 845-851. doi:10.1007/s11661-000-1004-3.

[75] S.V. Razorenov, G.I. Kanel, G.V. Garkushin, O.N. Ignatova, Resistance to dynamic deformation and fracture of tantalum with different grain and defect structures, Phys. Solid State. 54 (2012) 790-797.

doi:10.1134/S1063783412040233.

[76] Q. An, R. Ravelo, T.C. Germann, W.Z. Han, S.-N.N. Luo, D.L. Tonks, I.I.I. W. A. Goddard, Shock compression and spallation of single crystal tantalum, AIP Conf. Proc. 1426 (2012) 1259-1262. doi:10.1063/1.3686509. 
[77] E.N. Hahn, T.C. Germann, R.J. Ravelo, J.E. Hammerberg, M.A. Meyers, Nonequilibrium molecular dynamics simulations of spall in single crystal tantalum, in: AIP Publishing, 2017. doi:10.1063/1.4971594.

[78] G. Roy, Vers une modélisation approfondie de l'endommagement ductile dynamique: investigation expérimentale d'une nuance de tantale et développements théoriques, Ph.D. Thesis, Poitiers, 2003.

[79] J.C.F. Millett, G. Whiteman, N.T. Park, S. Case, N.K. Bourne, The role of cold work on the shock response of tantalum, J. Appl. Phys. 113 (2013) 233502. doi:10.1063/1.4810896.

[80] G.T. Gray III, N.K. Bourne, V. Livescu, C.P. Trujillo, S. MacDonald, P. Withers, The influence of shock-loading path on the spallation response of Ta, J. Phys. Conf. Ser. 500 (2014) 112031. doi:10.1088/1742-6596/500/11/112031.

[81] E.N. Hahn, T.C. Germann, R. Ravelo, J.E. Hammerberg, M.A. Meyers, On the ultimate tensile strength of tantalum, Acta Mater. 126 (2017) 313-328. doi:10.1016/j.actamat.2016.12.033.

[82] S. Christy, H. Pak, M. Meyers, Metallurgical applications of shock wave and high-strain-rate phenomena, N. Y. Basel. (1986).

[83] G.I. Kanel, Spall fracture: methodological aspects, mechanisms and governing factors, Int. J. Fract. 163 (2010) 173-191. doi:10.1007/s10704-009-9438-0.

[84] G. Kanel, S. Rasorenov, V. Fortov, The dynamic strength of copper single crystals, Dekker, New York, 1992.

[85] R.W. Minich, J.U. Cazamias, M. Kumar, A.J. Schwartz, Effect of microstructural length scales on spall behavior of copper, Metall. Mater. Trans. A. 35 (2004) 2663-2673. doi:10.1007/s11661-004-0212-7.

[86] E.M. Bringa, S. Traiviratana, M.A. Meyers, Void initiation in fcc metals: Effect of loading orientation and nanocrystalline effects, Acta Mater. 58 (2010) 44584477. doi:10.1016/j.actamat.2010.04.043.

[87] V.A. Lubarda, M.S. Schneider, D.H. Kalantar, B.A. Remington, M.A. Meyers, Void growth by dislocation emission, Acta Mater. 52 (2004) 1397-1408. doi:10.1016/j.actamat.2003.11.022.

[88] T.P. Remington, C.J. Ruestes, E.M. Bringa, B.A. Remington, C.H. Lu, B. Kad, M.A. Meyers, Plastic deformation in nanoindentation of tantalum: A new mechanism for prismatic loop formation, Acta Mater. 78 (2014) 378-393. doi:10.1016/j.actamat.2014.06.058.

[89] Y. Tang, E.M. Bringa, M.A. Meyers, Ductile tensile failure in metals through initiation and growth of nanosized voids, Acta Mater. 60 (2012) 4856-4865. doi:10.1016/j.actamat.2012.05.030.

[90] J.R. Rice, R. Thomson, Ductile versus brittle behaviour of crystals, Philos. Mag. 29 (1974) 73-97. doi:10.1080/14786437408213555.

[91] J. Weertman, Dislocation based fracture mechanics, World Scientific, 1996.

[92] A.L. Stevens, L. Davison, W.E. Warren, Spall fracture in aluminum monocrystals: a dislocation-dynamics approach, J. Appl. Phys. 43 (1972) 4922-4927. doi:10.1063/1.1661046.

[93] Y. Tang, E.M. Bringa, M.A. Meyers, Inverse Hall-Petch relationship in nanocrystalline tantalum, Mater. Sci. Eng. A. 580 (2013) 414-426. doi:10.1016/j.msea.2013.05.024. 
[94] L.P. Kubin, G. Canova, M. Condat, B. Devincre, V. Pontikis, Y. Bréchet, Dislocation microstructures and plastic flow: a 3D simulation, in: Solid State Phenom., Trans Tech Publ, 1992: pp. 455-472.

[95] M. Tang, L.P. Kubin, G.R. Canova, Dislocation mobility and the mechanical response of b.c.c. single crystals: A mesoscopic approach, Acta Mater. 46 (1998) 3221-3235. doi:10.1016/S1359-6454(98)00006-8.

[96] H.M. Zbib, T. Diaz de la Rubia, A multiscale model of plasticity, Int. J. Plast. 18 (2002) 1133-1163. doi:10.1016/S0749-6419(01)00044-4.

[97] M.A. Shehadeh, H.M. Zbib, T. Diaz de la Rubia, Multiscale dislocation dynamics simulations of shock compression in copper single crystal, Int. J. Plast. 21 (2005) 2369-2390. doi:10.1016/j.ijplas.2004.12.004.

[98] G.J. Cheng, M.A. Shehadeh, Dislocation behavior in silicon crystal induced by laser shock peening: A multiscale simulation approach, Scr. Mater. 53 (2005) 1013-1018. doi:10.1016/j.scriptamat.2005.07.014.

[99] G.J. Cheng, M.A. Shehadeh, Multiscale dislocation dynamics analyses of laser shock peening in silicon single crystals, Int. J. Plast. 22 (2006) 2171-2194. doi:10.1016/j.ijplas.2006.03.006.

[100] M.A. Shehadeh, H.M. Zbib, On the homogeneous nucleation and propagation of dislocations under shock compression, Philos. Mag. 96 (2016) 2752-2778. doi:10.1080/14786435.2016.1213444.

[101] N. Thadhani, M. Meyers, Kinetics of martensitic transformation induced by a tensile stress pulse, Acta Metall. 34 (1986) 1625-1641.

[102] S.-N. Chang, M.A. Meyers, Martensitic transformation induced by a tensile stress pulse in Fe-22.5 wt\% Ni-4wt\% Mn alloy, Acta Metall. 36 (1988) 10851098.

[103] Y. Sano, S. Chang, M. Meyers, S. Nemat-Nasser, Identification of stress-induced nucleation sites for martensite in Fe-31.8 wt\% Ni-0.02 wt\% C alloy, Acta Metall. Mater. 40 (1992) 413-417.

[104] D.H. Kalantar, J.F. Belak, G.W. Collins, J.D. Colvin, H.M. Davies, J.H. Eggert, T.C. Germann, J. Hawreliak, B.L. Holian, K. Kadau, P.S. Lomdahl, H.E. Lorenzana, M.A. Meyers, K. Rosolankova, M.S. Schneider, J. Sheppard, J.S. Stölken, J.S. Wark, Direct observation of the $\alpha-\varepsilon$ transition in shock-compressed iron via nanosecond X-ray diffraction, Phys. Rev. Lett. 95 (2005) 75502. doi:10.1103/PhysRevLett.95.075502.

[105] C.-H. Lu, E.N. Hahn, B.A. Remington, B.R. Maddox, E.M. Bringa, M.A. Meyers, Phase Transformation in Tantalum under Extreme Laser Deformation, Sci. Rep. 5 (2015) 15064. doi:10.1038/srep15064.

[106] L.M. Hsiung, D.H. Lassila, Shock-Induced Omega Phase in Tantalum, Scr. Mater. 38 (1998) 1371-1376. doi:10.1016/S1359-6462(98)00049-9.

[107] L.M. Hsiung, D.H. Lassila, Shock-induced displacive transformations in tantalum and tantalum-tungsten alloys, Scr. Mater. 39 (1998) 603-609. doi:10.1016/S1359-6462(98)00203-6.

[108] T. Kumagai, S. Izumi, S. Hara, S. Sakai, Development of bond-order potentials that can reproduce the elastic constants and melting point of silicon for classical molecular dynamics simulation, Comput. Mater. Sci. 39 (2007) 457464. doi:10.1016/j.commatsci.2006.07.013. 
[109] S. Zhao, B. Kad, E.N. Hahn, B.A. Remington, C.E. Wehrenberg, C.M. Huntington, H.-S. Park, E.M. Bringa, K.L. More, M.A. Meyers, Pressure and shear-induced amorphization of silicon, Extreme Mech. Lett. 5 (2015) 74-80. doi:10.1016/j.eml.2015.10.001.

[110] S. Zhao, E.N. Hahn, B. Kad, B.A. Remington, C.E. Wehrenberg, E.M. Bringa, M.A. Meyers, Amorphization and nanocrystallization of silicon under shock compression, Acta Mater. 103 (2016) 519-533. doi:10.1016/j.actamat.2015.09.022.

[111] C.H. Lu, B.A. Remington, B.R. Maddox, B. Kad, H.S. Park, M. Kawasaki, T.G. Langdon, M.A. Meyers, Laser compression of nanocrystalline tantalum, Acta Mater. 61 (2013) 7767-7780. doi:10.1016/j.actamat.2013.09.016.

[112] M.A. Meyers, M.S. Schneider, H. Jarmakani, B. Kad, B.A. Remington, D.H. Kalantar, J. McNaney, B. Cao, J. Wark, Deformation Substructures and Their Transitions in Laser Shock-Compressed Copper-Aluminum Alloys, Metall. Mater. Trans. A. 39 (2008) 304-321. doi:10.1007/s11661-007-9359-3.

\section{Figure Captions}

Figure 1. a) Experimental assembly to generate an HE-induced shock in metal by direct explosive detonation causing a grazing shock wave propagation; b) Setup of a shock recovery experiment using the acceleration of a flyer plate by an explosive charge; c) typical schematic of a gas gun configuration producing shock compression experiments. Adapted from Meyers et al. [5].

Figure 2. Methods of laser shocking materials; (a) direct laser illumination at an intensity above the ablation threshold; (b) laser irradiation through a transparent overlay to increase achievable pressures; (c) laser accelerated flyer plate;(d) use of reservoir create more uniform pressure distribution and (iv) laser generated X-rays through a hohlraum (indirect drive). Adapted from Meyers et al. 2009 [5].

Figure 3. Direct drive laser ablation of a target. The pulsed laser arrives from the left hand side and interacts with the surface of the material by exciting electrons. As the temperature increases the material may melt or ablate. As the material expands it sends a 
shock wave into the target as a result of the reaction force, the speed of which can be determined using the rocket effect and pressure as determined by Lindl et al. [14].

Figure 4. Two different types of shock wave configurations that can be produced: a) trapezoidal shock wave propagation created by a flyer plate impact; and b) triangular shock wave propagation created by a pulsed laser irradiation. Adapted from Cao et al. [17].

Figure 5. Change in pressure pulse shape as a function of distance for laser-generated shock calculated by HYADES. (a) laser energy of $70 \mathrm{~K}$; (b) $200 \mathrm{~J}$; (c) $300 \mathrm{~J}$; (d) pressure decay for three laser energy levels. From Schneider et al. [18].

Figure 6. The three main laser facilities used for laser shock compression experiments: a) schematic of the interior laser bay design of the National Ignition Facility, Lawrence Livermore National Laboratory (LLNL) [19], with permission from LLNL; b) photo of the Jupiter Laser Facility, LLNL with permission from LLNL; and c) the Omega laser facility at Rochester University, NY with 60 laser beams [19], with permission for the Laboratory for laser Energetics, U. of Rochester.

Figure 7. A recovery tube housing a sample illuminated by multiple lasers simultaneously within a laser target chamber. A second target was illuminated with the same laser irradiation conditions using different beams, to measure the drive (pressure vs. time) with a time resolved VISAR diagnostic.

Figure 8. Detailed cross-section of the recovery tube fixture for high strain rate laser driven shock experiments at the Omega laser facility, LLE.

Figure 9. The target assembly for one variation of the laser driven shock experiments consists of the Ta sample target mounted on a washer offset by a vacuum gap from the "drive package". The drive package consists of a polycarbonate ablator that the laser irradiates, driving a shock through the $\mathrm{CH}(2 \% \mathrm{Br})$ reservoir. At shock breakout, the reservoir releases across the vacuum gap then stagnates on the Ta sample, launching a compression wave which quickly steepens into a shock. Adapted from Lu et al. [24,111].

Figure 10. Crater formation from laser compression in tantalum: a) SEM image of single crystalline tantalum after laser shock; and b) the profilometry of the crater depth. From Lu et al. [24].

Figure 11. Voids and bubbles due to melting and vaporization from laser compression. From Maddox et al. [23].

Figure 12. Solid-state lattice response under shock driven compression. A set-up for using time resolved diffraction to simultaneously measure the lattice compression in the direction of the shock velocity (labeled Bragg in the figure), and the lattice response 
perpendicular to the direction of the shock velocity (labeled Laue), using a laser drive and a pulsed x-ray source at the Omega laser.

Figure 13. Schematic representation of distortions caused in an ideal lattice due to a planar shock passing through the lattice from top to bottom in the figure; (a) original configuration of planes; (b) elastic compression, prior to the onset of plasticity; (c) plastic response with the lattice compression parallel to shock velocity larger than the compression perpendicular to the shock velocity, with the difference determined by the material strength of the lattice; (d) hydrostatic compression, when the compressions parallel and perpendicular are equal, as would be expected if there were no material strength. From Meyers et al. [28].

Figure 14. X-ray diffraction: data from Bragg (reflection) and Laue (transmission) diagnostics. Flash X-ray diffraction was used, typical results for copper (200) and (020) planes are shown in the Bragg (left) and Laue (right); a) time-integrated and b) timeresolved data. The shock was driven into this $2 \mu \mathrm{m}$ thick single crystal $\mathrm{Cu}$ sample along the [200] direction. From Meyers et al. [28].

Figure 15. Copper shear strength results from the diffraction data from shock compression shown in Fig. 14. Adapted from Meyers et al. [28].

Figure 16. Highest strain rate for [001] $\mathrm{Cu}$ was found to be at a shock strength of $\sim 100$ GPa. From Murphy et al. [33].

Figure 17. (a) Schematics of dynamic $X$ ray diffraction setup showing simultaneous shock pulse propagation and diffraction of $X$ rays and providing both time resolved and time integrated change in the diffraction angles due to changes in lattice parameter and/or appearance of a new phase; (b) Intersection of diffraction cones with recording plane for iron shocked at a pressure of $26 \mathrm{GPa}$, above the threshold for the alpha to epsilon transition $(=13 \mathrm{GPa})$. The diffraction lines from the static crystal are marked by squares, the ones from uniaxial compression by laser by triangles, and the lines from the epsilon phase are crosses. The shift in diffraction angle with pressure as well as the new lines provide a wealth of information on the extreme state accessed by shock compression. From Kalantar et al. [34].

Figure 18. (a) Experimental setup for determination of ripple growth in Va sample as a function of time; (b) schematic representation of ripple evolution with and without material strength; (c) growth of waviness on surface of tantalum specimen under the effect of shock compression for different constitutive equations: SG: Steinberg-Guinan; PTW: Preston-Tonks-Wallace; SL: Steinberg Lund; LMS: Livermore Multi Scale; it is clear that growth is independent of grain size. If strength is zero, the growth is highest; if strength is infinite, growth is zero. By dialing the appropriate strength parameters into constitutive equation one can match experimental and calculated results and extract a strength. From Park et al. [35].

Figure 19. Dislocation interface in homogeneous generation model. 
Figure 20. Experimental results by Murr, Kuhlmann-Wilsdorf, Meyers and Trueb for dislocation density compared with theoretical predictions for both dislocation motion at the shear wave speed and stationary dislocations. From Meyers et al. [5].

Figure 21. a) Experimental observation under TEM of dislocation loops in laser shocked copper, the different loop sizes $(1=$ large; $\mathrm{s}=$ small $)$ and shapes $(\mathrm{e}=$ elongated $)$ are indicated(Adapted from Meyers et al. [96]); b) laser shock compression of nickel generated stacking-faults; c) elastic and plastic compression, where dislocation loops are generated in the regions showing plastic relaxation. Adapted from Meyers et al. [112].

Figure 22. Face-centered cubic metals: generation of partial dislocation loops; a) Schematic showing dislocation loops nucleating at slip planes behind the shock front, shown in red with propagation along [001] Adapted from [5]; b) Stacking fault sets are marked as A, B, C, and D, where set A exhibits the highest density of occurrence (laser energy 205 J). From Schneider et al. [18] .

Figure 23. MD simulations showing peak applied stress ( $\sigma_{z z}$, equivalent to $\sigma_{11}$ in paper) during shock compression of nickel along [001], the lattice $\mathrm{z}$ direction.

Figure 24. Holian-Lomdahl plot showing plasticity $\left(a_{o} / l\right)$ vs. shock strength $\left(U_{p} / C_{o}\right)$ in the as-shocked and unloaded conditions.

Figure 25. Schematic representation of a shock front traveling through a bcc metal in the [001] direction; a) dislocation loops nucleating at the slip planes have Burgers vectors parallel to the slip plane direction; b) shear loops nucleating on the $\{211\}$ planes in shock compression have Burgers vectors intersecting the slip plane directions.

Figure 26. Predictions of dislocation velocity as a function of stress from the results of Tang et al. [49] and Deo et al. [50]. Adapted from Lu et al. [24].

Figure 27. Homogeneous dislocation generation vs. dislocation multiplication in tantalum, a bcc metal. Experimental results from Gray and Vecchio [59], Hsiung [60], and $\mathrm{Lu}$ et al. [24] are plotted alongside models of dislocation multiplication and homogeneous nucleation.

Figure 28. Recovery of single crystalline tantalum after laser shock compression revealed twins under TEM observation. From Lu et al. [24].

Figure 29. The slip-twinning transition for tantalum under laser compression is inferred to happen at a critical shock strength, above which slip cannot relieve the shock induced shear stress rapidly enough, and twinning commences (to relieve the shear stress). From Lu et al. [24]. 
Figure 30. Dislocation density experimentally deduced from TEM results of recovered shocked samples.

Figure 31. Theoretical and experimental spall strengths for well known fcc and bcc metals. Adapted from Jarmakani et al. [68].

Figure 32. Effect of strain rate on spall strength of tantalum; experimental and molecular dynamics predictions results from several sources. Strain rate of $10^{13} \mathrm{~s}^{-1}$, corresponding to atomic frequency can be considered ultimate value and gives spall strength of $\sim 35 \mathrm{GPa}$ close to Grady's theoretical cleavage stress (43 GPa). Adapted from Hahn et al. [81].

Figure 33. Elongated void in copper with high density of surrounding dislocations.

Figure 34. Experimentally observed dislocation activity (slip) around growing voids; b) optical micrograph of a slip band initiating from a void.

Figure 35. Schematic showing tensile strength and grain-boundary strength for copper as a function of strain rate; single crystal, large grain size and small grain size polycrystals shown.

Figure 36. Spall strength, measured by pullback velocity, as a function of shock pressure for copper with different grain sizes: 8,45 , and $90 \mu \mathrm{m}$ as well as $\mathrm{Cu}$ with the addition of $\mathrm{SiO}_{2}$ particles and monocrystalline $\mathrm{Cu}$ with three orientations. The presence of grain boundaries and second phase particle provides initiation sites for void initiation. From Minich et al. [85].

Figure 37. Representation of two slip planes intersecting a void at $45^{\circ}$ angle. Loading on the [110] axis (z-axis).

Figure 38. a) Schematic and b) MD simulation showing tripolar loops due to uniaxial loading of a spherical void in $\mathrm{Cu}$, along the [111] axis. Adapted from Bringa et al. [86].

Figure 39. Molecular dynamics sequence showing shear and prismatic loop formation in single crystalline tantalum for high strain rates $\left(\sim 10^{8} \mathrm{~s}^{-1}\right)$ under hydrostatic tension.

Figure 40. Molecular dynamics sequence showing shear loop formations in single crystalline tantalum for high strain rates $\left(\sim 10^{8} \mathrm{~s}^{-1}\right)$ under uniaxial compression.

Figure 41. Representation of dislocation emission from a void for uniaxial tension: a) top view; and b) side view showing shear loop emission.

Figure 42. Stress, scaled with shear modulus, versus void size (void radius scaled with Burgers vector), for both fcc and bcc metals, including MD simulation results and analytical predictions. 
Figure 43. Plasticity in bec tantalum under uniaxial strain along [001] at a high strain rate of $10^{9} \mathrm{~s}^{-1}$; a) tri-vacancy at strain of $0 \%$; and b) stacking faults that nucleated from the trivacancy at strain of $11 \%$.

Figure 44. a) Experimental evidence of void initiation, with deviation from spherical shapes resulting from the anisotropy of plastic deformation. Square/rectangular shapes observed in (a) Ni, (b) $\mathrm{Cu}$, and (c) Al (Stevens et al. [92]); ; and (d) sequence showing void growth in monocrystalline $\mathrm{Cu}$ under uniaxial tension. The light blue lines correspond to stacking faults, which are bounded by partial dislocations.

Figure 45. Simulation showing nanocrystalline Ta with an average grain size of $27 \mathrm{~nm}$, undergoing uniaxial tension; a) the arrows on the right and left signify the direction of tension; b) circled in red is the initiation of a void; c) void growth and coalescence leads to crack formation and propagation; and d) twinning is observed inside one of the grains, as circled in red.

Figure 46. Schematic of a nanocrystalline bcc metal undergoing uniaxial tension; a) a void preferentially nucleates at the grain boundary; b) voids coalesce along grain boundaries; and c-d) at the grain boundary, crack induced twinning.

Figure 47. MD sequence showing spalling in nanocrystalline tantalum. Notice plastic deformation around growing voids, in contrast with situation in Fig. 45 and 46, where tension is applied uniformly on a nanocrystalline specimen without defects. Quasiisentropic compression followed by tension. The compressive strain rate is $10^{9} \mathrm{~s}^{-1}$ and tensile strain rate is $10^{8} \mathrm{~s}^{-1}$. Pre-compression leads to much more realistic grain boundary and defect structures. This in turn dictates that tensile failure is much more ductile including multiple dislocation and twin emissions that can be seen in(c) and (d). Adapted from Hahn et al. [81].

Figure 48. Sequence showing how an initially roughly spherical nanosized void develops facets as it grows; also notice associated emission of shear loops at void corners.

Figure 49. (a-c) Snapshots generated by dislocation dynamics (DD) simulations in copper subjected to shock compression at times of 67, 90, and 120 ps. Pressure of 35 GPa, 50 ps rise time shock wave. Loops nucleate homogeneously as the wave travels through copper from left to right, while those previously nucleated grow as the crystal relaxes. (d) stresses and strains as a function of time; notice that stress state relaxes to hydrostatic as dislocations are nucleated and move; (e) dislocation density as a function of pressure from DD and MD simulations compared to experimental results. Adapted from Shehadeh et al. [97].

Figure 50. Shock/shear induced transformation in tantalum observed after a $\sim 70 \mathrm{GPa}$ laser-induced pulse with an initial duration of $3 \mathrm{~ns}$ (a, b, c). Dark-field TEM images showing $\omega$ phase (bright areas) for shock orientations of a) [110], b) [111], c) [123]; d) MD simulation showing the hexagonal phase forming as nodules within the bcc structure of tantalum; and e) detail of a nodule with hexagonal structure. 
Figure 51. (a,b) Shock and shear-induced amorphization in silicon observed after pulsed laser compression at Omega Facility (LLE). C-Si designates crystalline $\mathrm{Si}$ whereas a-Si indicates the amorphous phase; and (c,d) MD simulations showing both the pressureinduced layer close to the energy deposition surface and the shear induced amorphization on right. From Zhao et al. [109]. 
a)

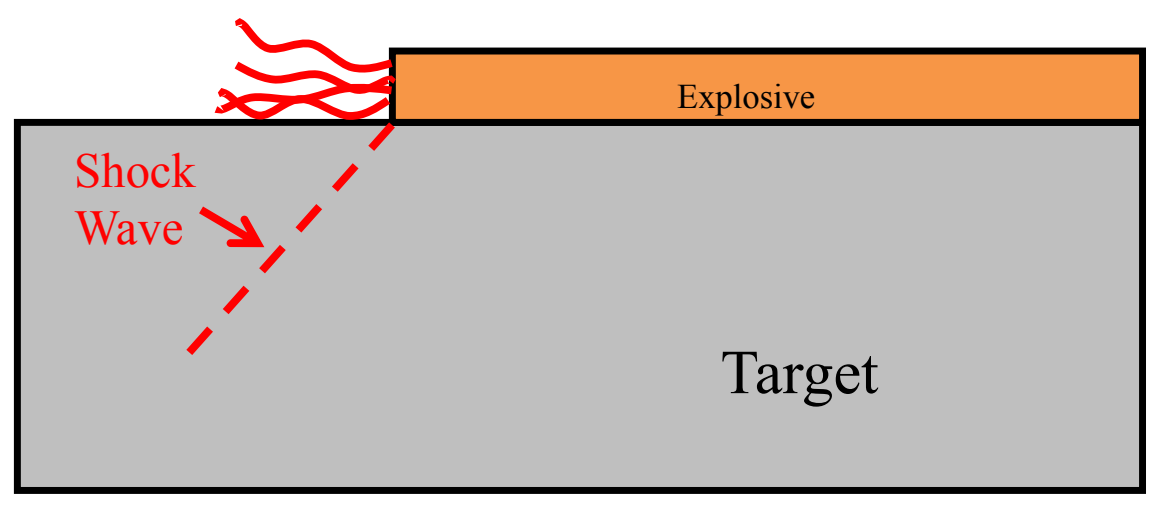

b)

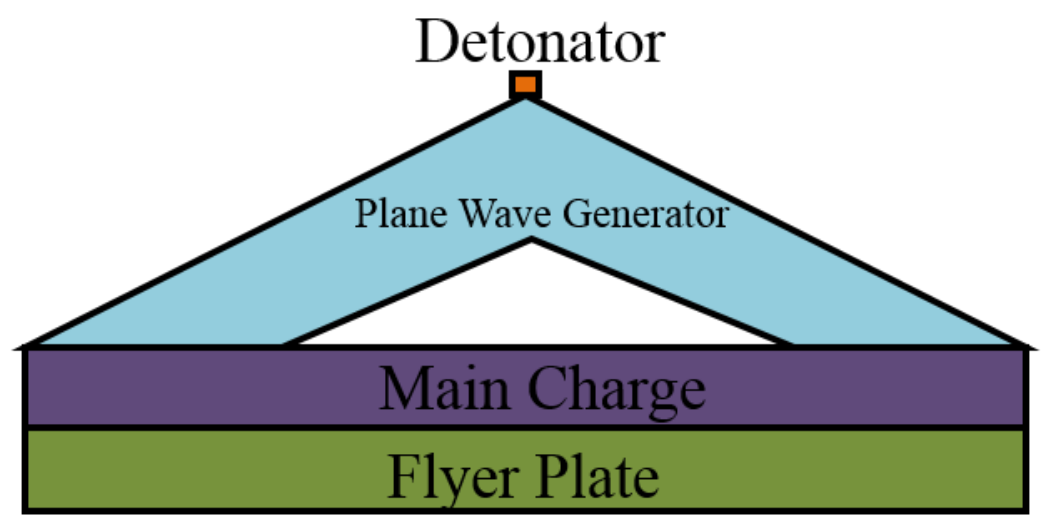

Target

c)

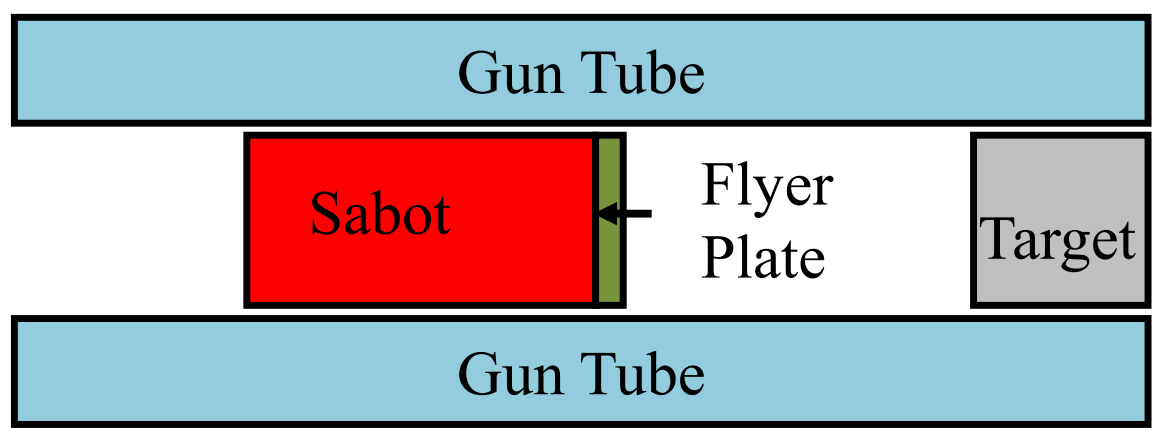

Figure 1. a) Experimental assembly to generate an HE-induced shock in metal by direct explosive detonation causing a grazing shock wave propagation; b) Setup of a shock recovery experiment using the acceleration of a flyer plate by an explosive charge; c) typical schematic of a gas gun configuration producing shock compression experiments. Adapted from Meyers et al. [5]. 
(a) LASER

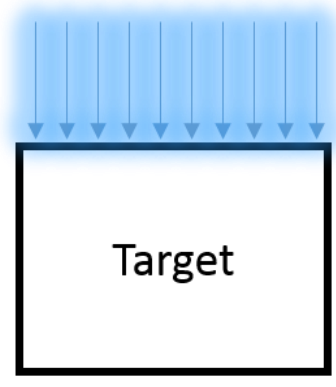

(c) LASER

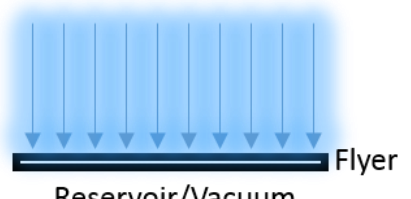

Reservoir/Vacuum

Target (b) LASER

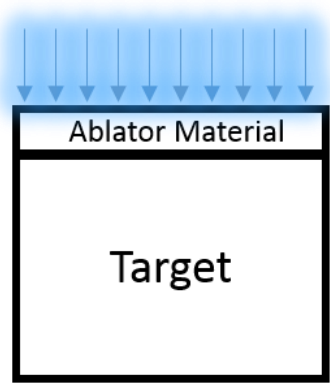

(d) LASER

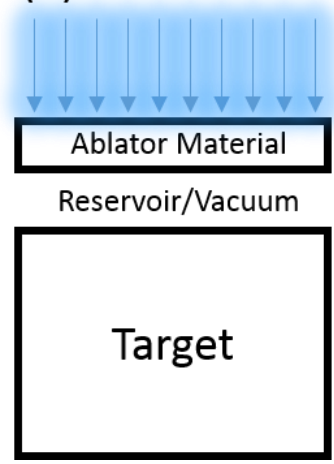

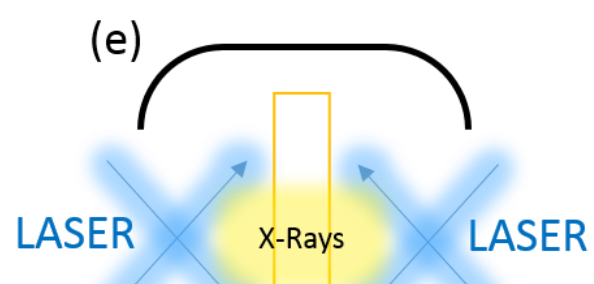

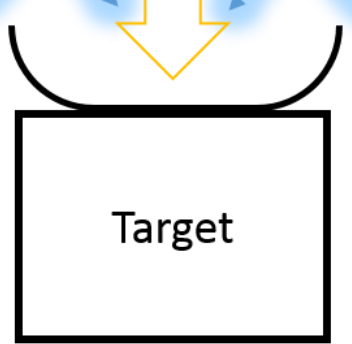

Figure 2. Methods of laser shocking materials; (a) direct laser illumination at an intensity above the ablation threshold; (b) laser irradiation through a transparent overlay to increase achievable pressures; (c) laser accelerated flyer plate;(d) use of reservoir create more uniform pressure distribution and (iv) laser generated X-rays through a hohlraum (indirect drive). Adapted from Meyers et al. 2009 [5]. 


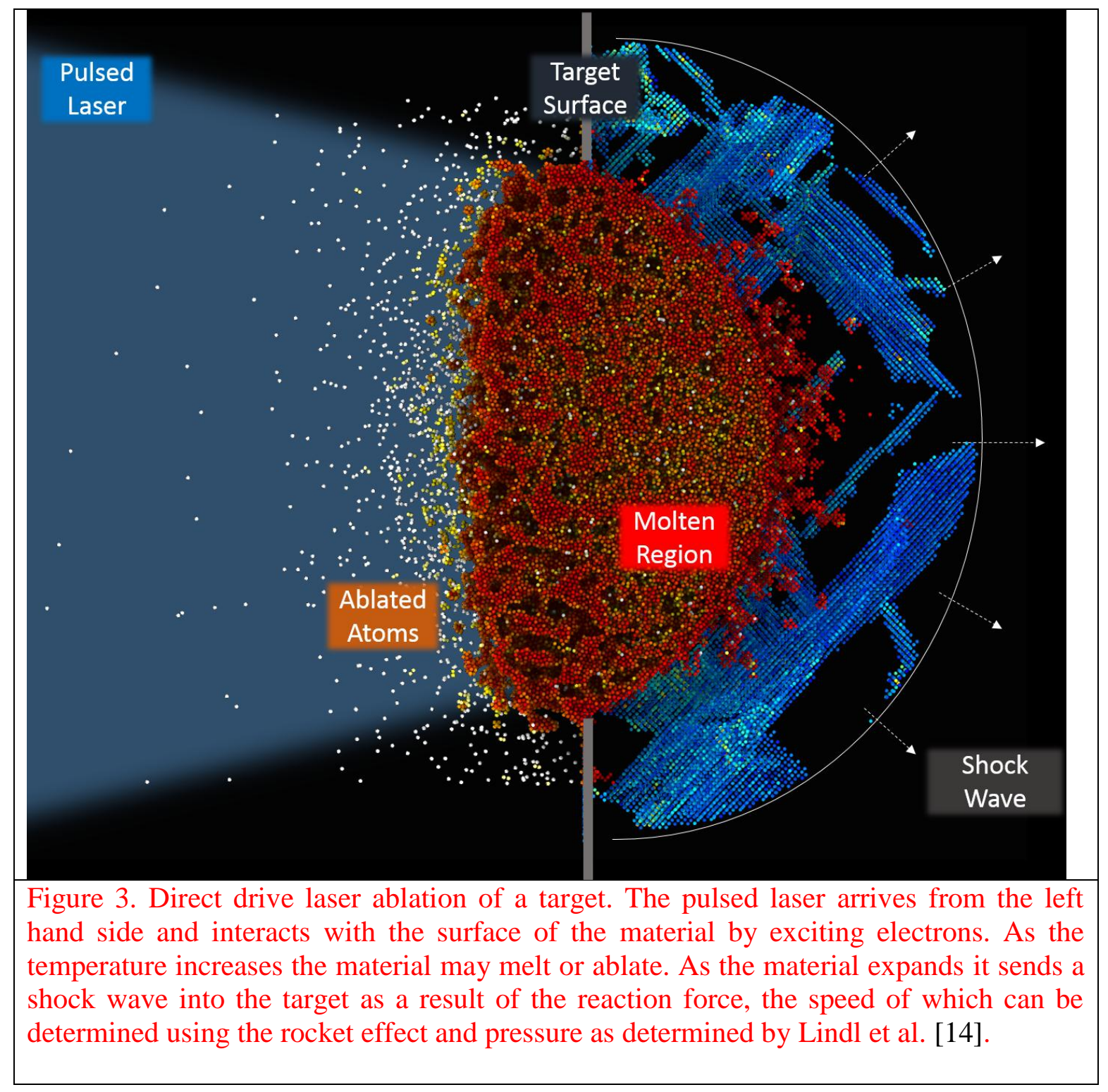


a)

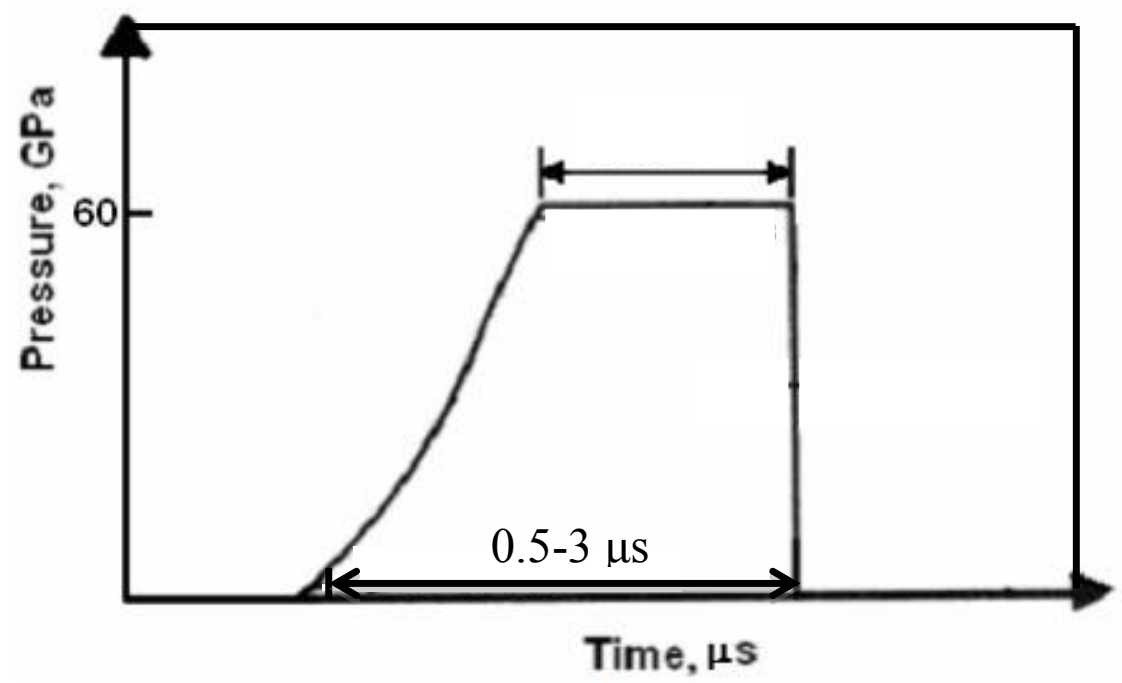

b)

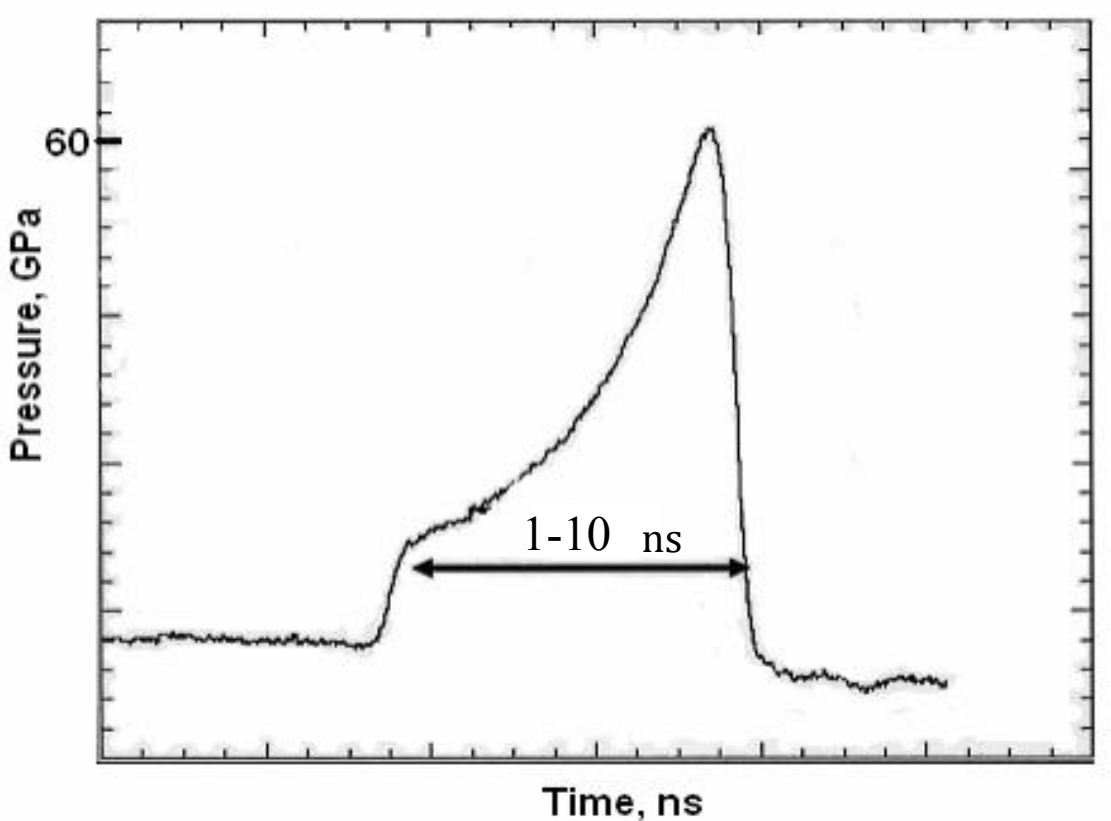

Figure 4. Two different types of shock wave configurations that can be produced: a) trapezoidal shock wave propagation created by a flyer plate impact; and b) triangular shock wave propagation created by a pulsed laser irradiation. Adapted from Cao et al. [17]. 


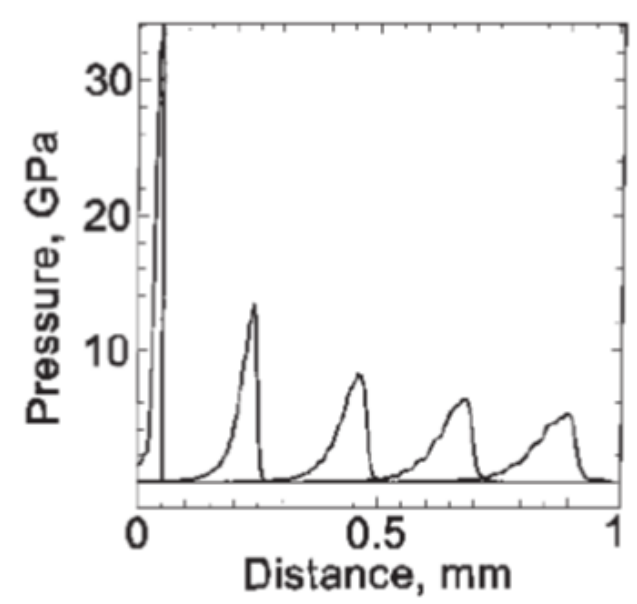

(a)

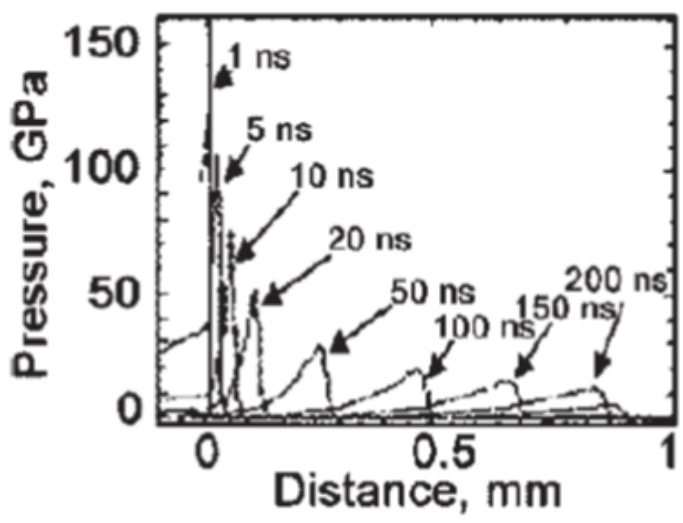

(c)

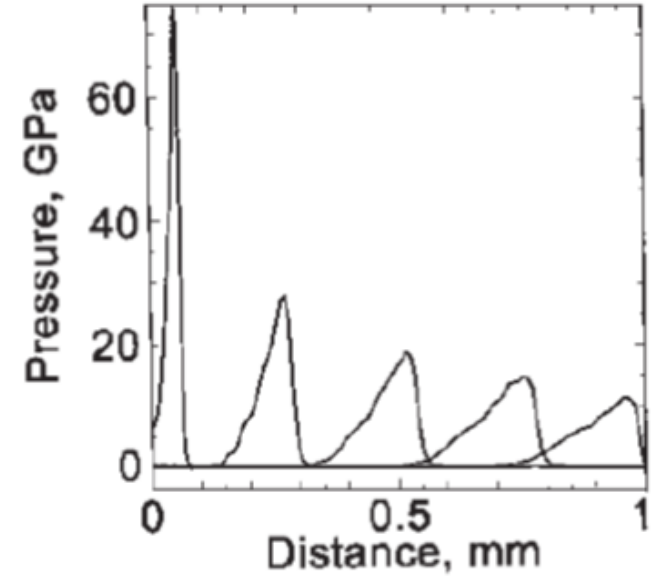

(b)

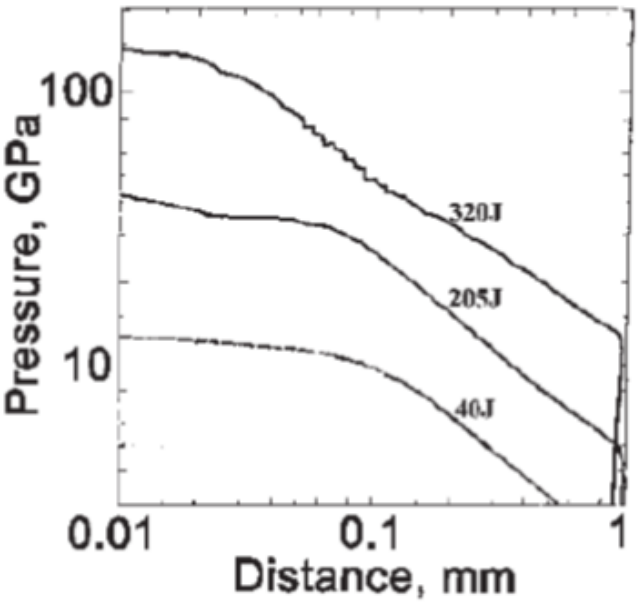

(d)

Figure 5. Change in pressure pulse shape as a function of distance for laser-generated shock calculated by HYADES. (a) laser energy of 70 K; (b) $200 \mathrm{~J}$; (c) $300 \mathrm{~J}$; (d) pressure decay for three laser energy levels. From Schneider et al. [18]. 


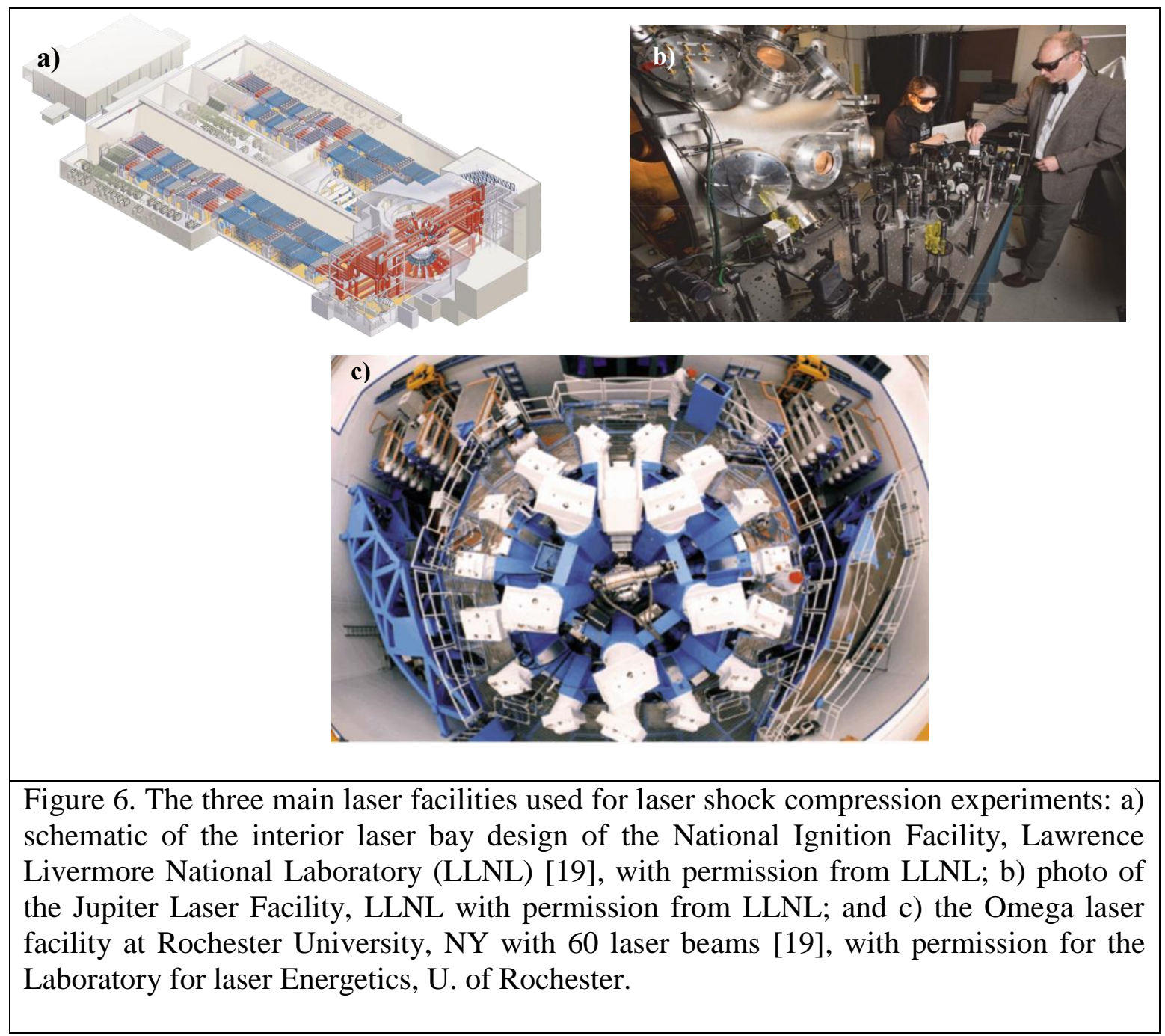




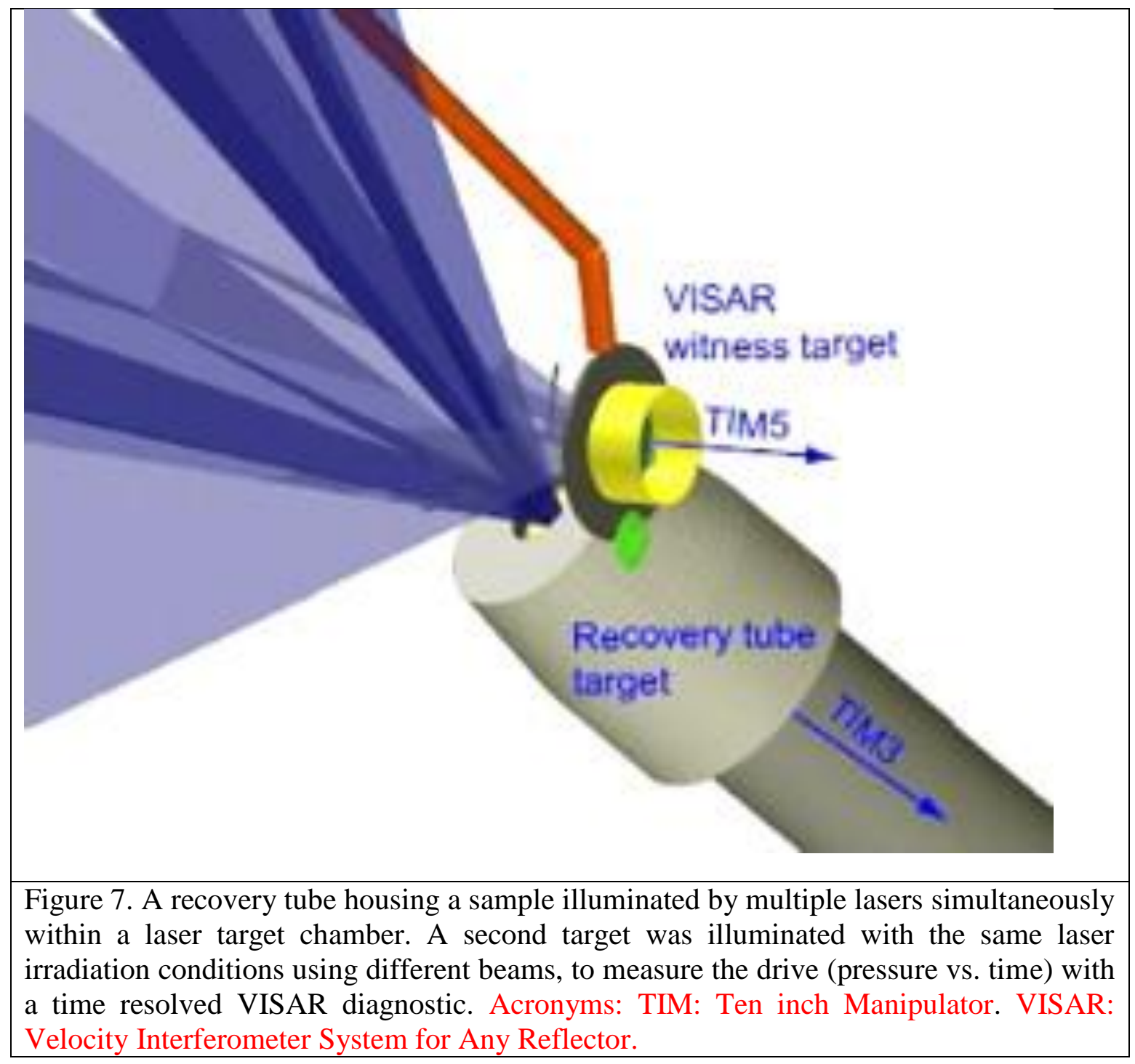



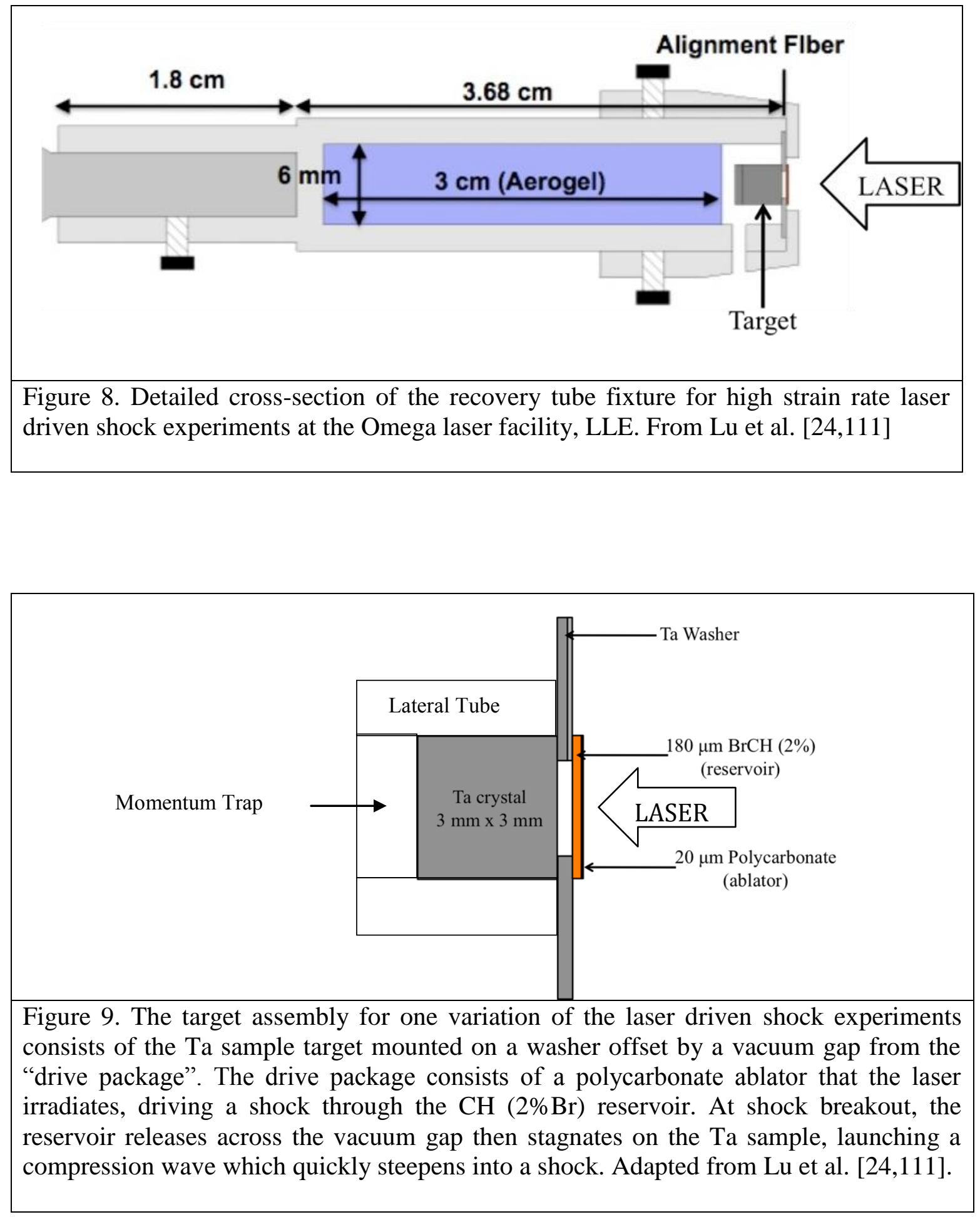
a)

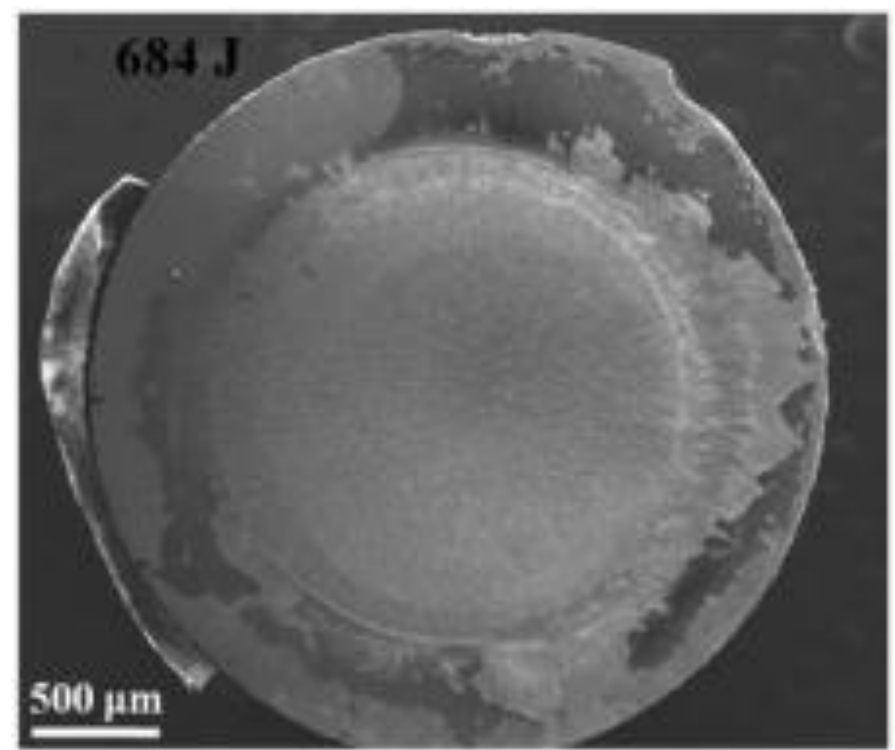

b)

\section{$684 \mathrm{~J}$}
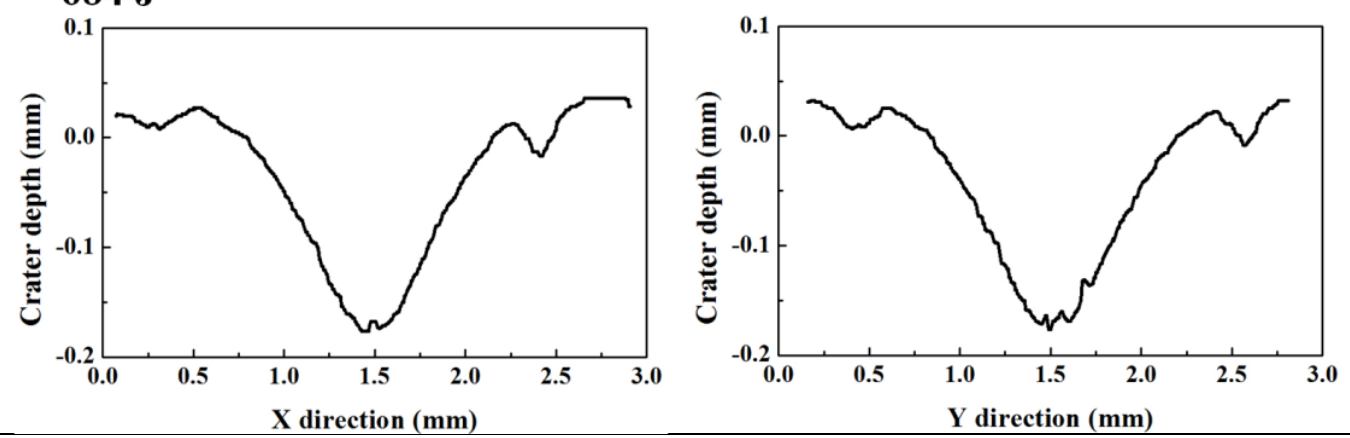

Figure 10. Crater formation from laser compression in tantalum: a) SEM image of single crystalline tantalum after laser shock; and b) the profilometry of the crater depth. From Lu et al. [24]. Initial pressure of ..GPa and duration of $3 \mathrm{~ns}$. 


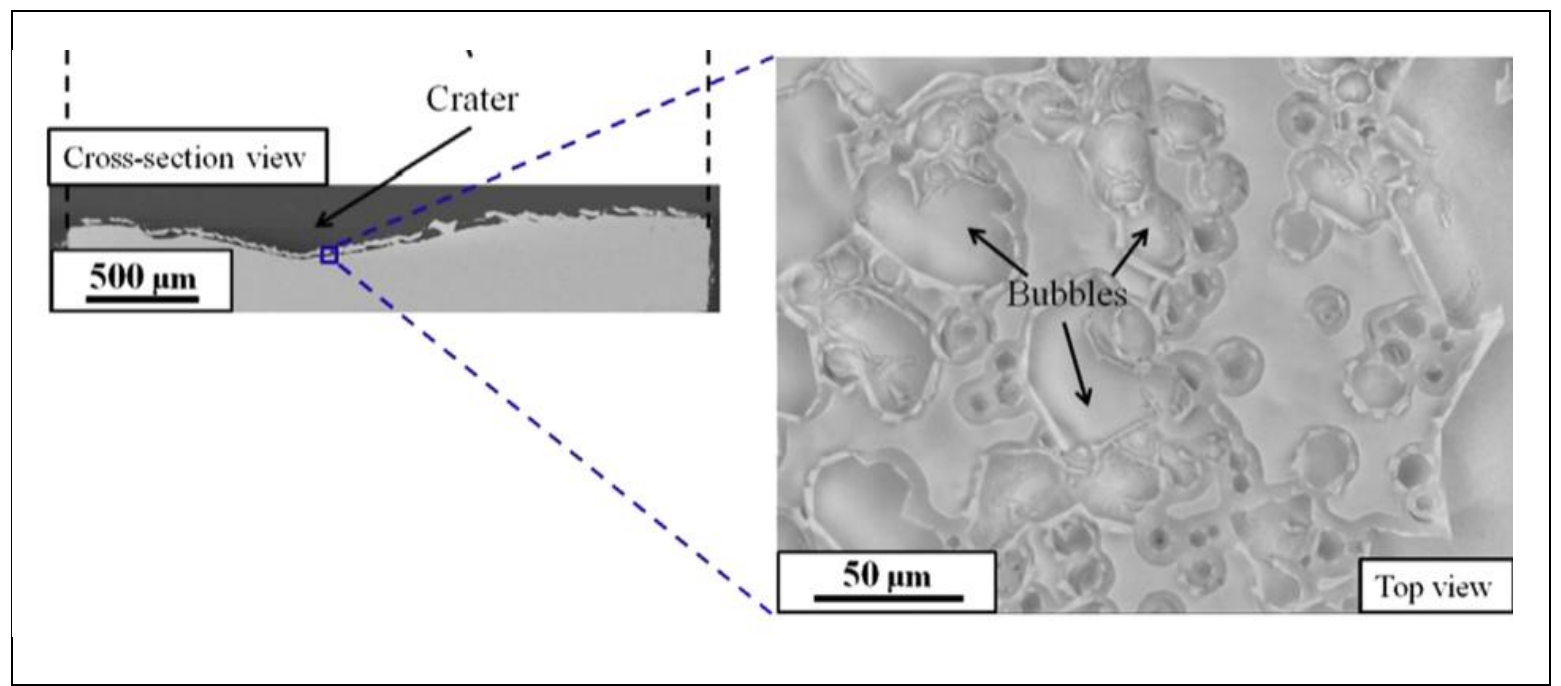

Figure 11. Voids and bubbles due to melting and vaporization from laser compression. From Maddox et al. [23].

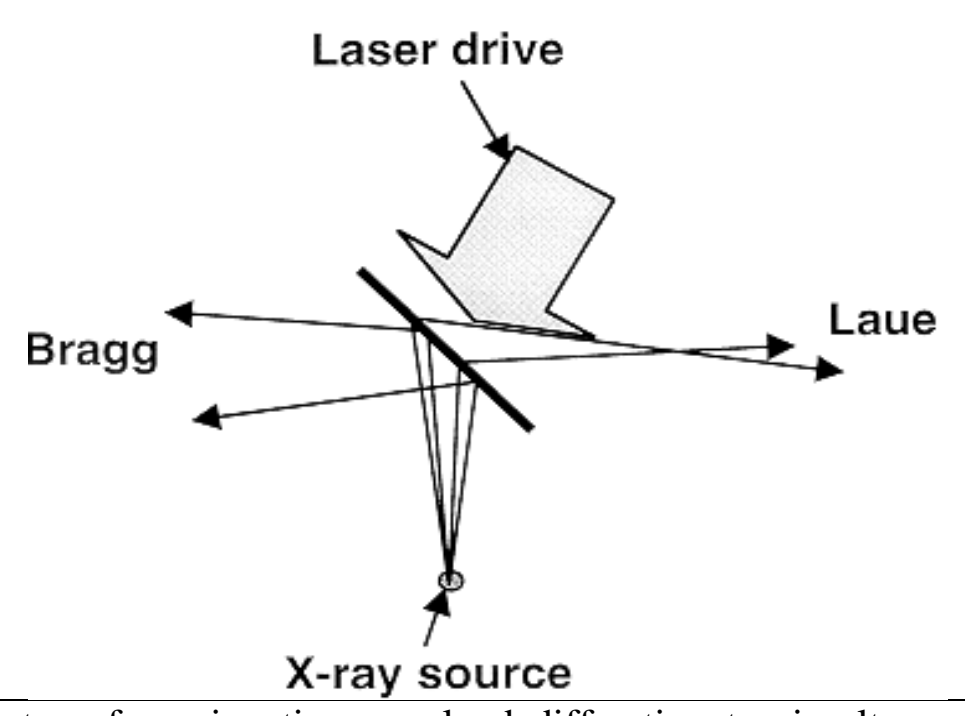

Figure 12 A set-up for using time resolved diffraction to simultaneously measure the lattice compression in the direction of the shock velocity (labeled Bragg in the figure), and the lattice response perpendicular to the direction of the shock velocity (labeled Laue), using a laser drive and a pulsed $\mathrm{x}$-ray source at the Omega laser. 
(a)

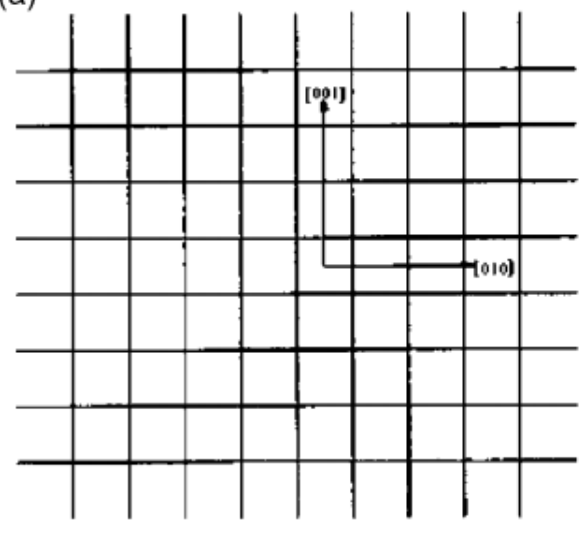

Reference Lattice

(c)

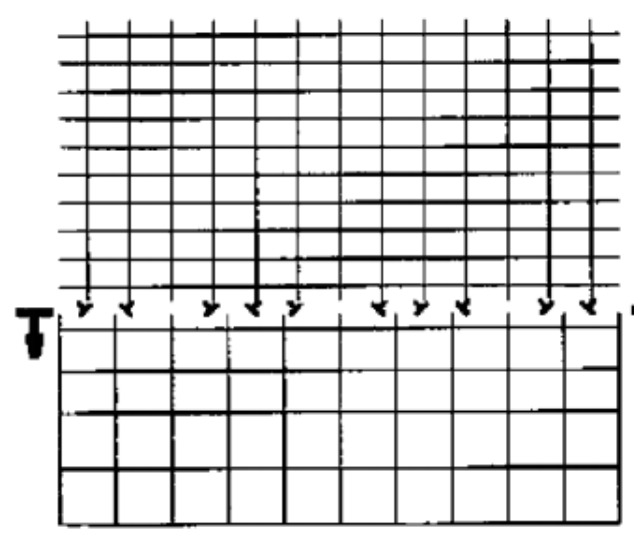

Elastic + Plastic (b)

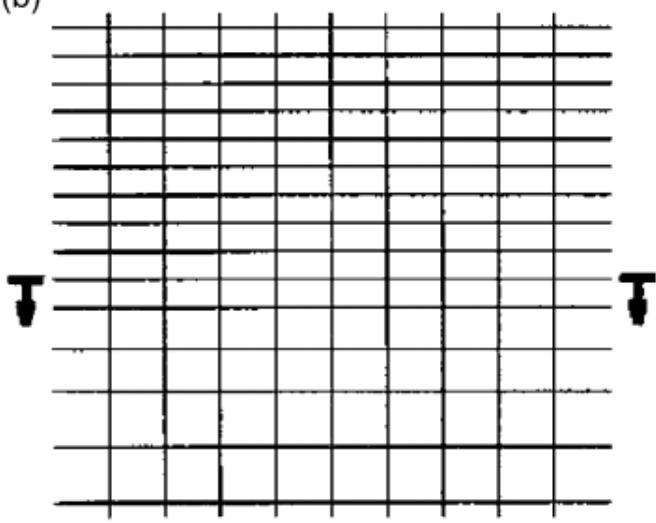

Elastic Distortion

(d)

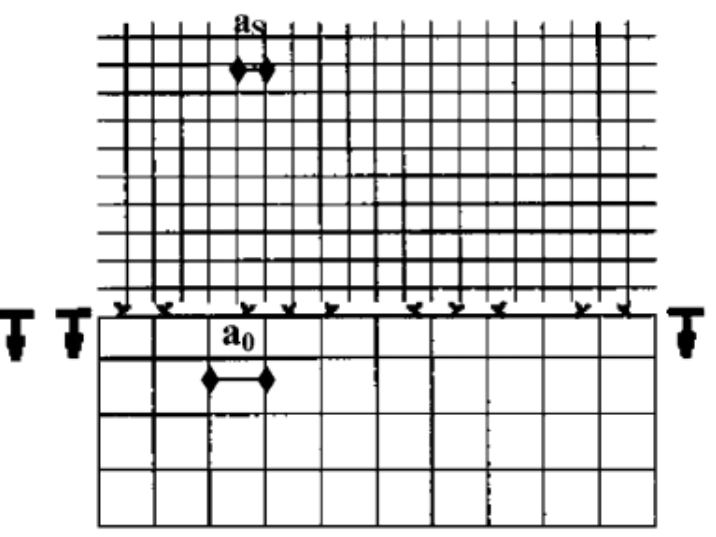

Hydrostatic Compression

Figure 13. Schematic representation of distortions caused in an ideal lattice due to a planar shock passing through the lattice from top to bottom in the figure; (a) original configuration of planes; (b) elastic compression, prior to the onset of plasticity; (c) plastic response with the lattice compression parallel to shock velocity larger than the compression perpendicular to the shock velocity, with the difference determined by the material strength of the lattice; (d) hydrostatic compression, when the compressions parallel and perpendicular are equal, as would be expected if there were no material strength. From Meyers et al. [28]. 


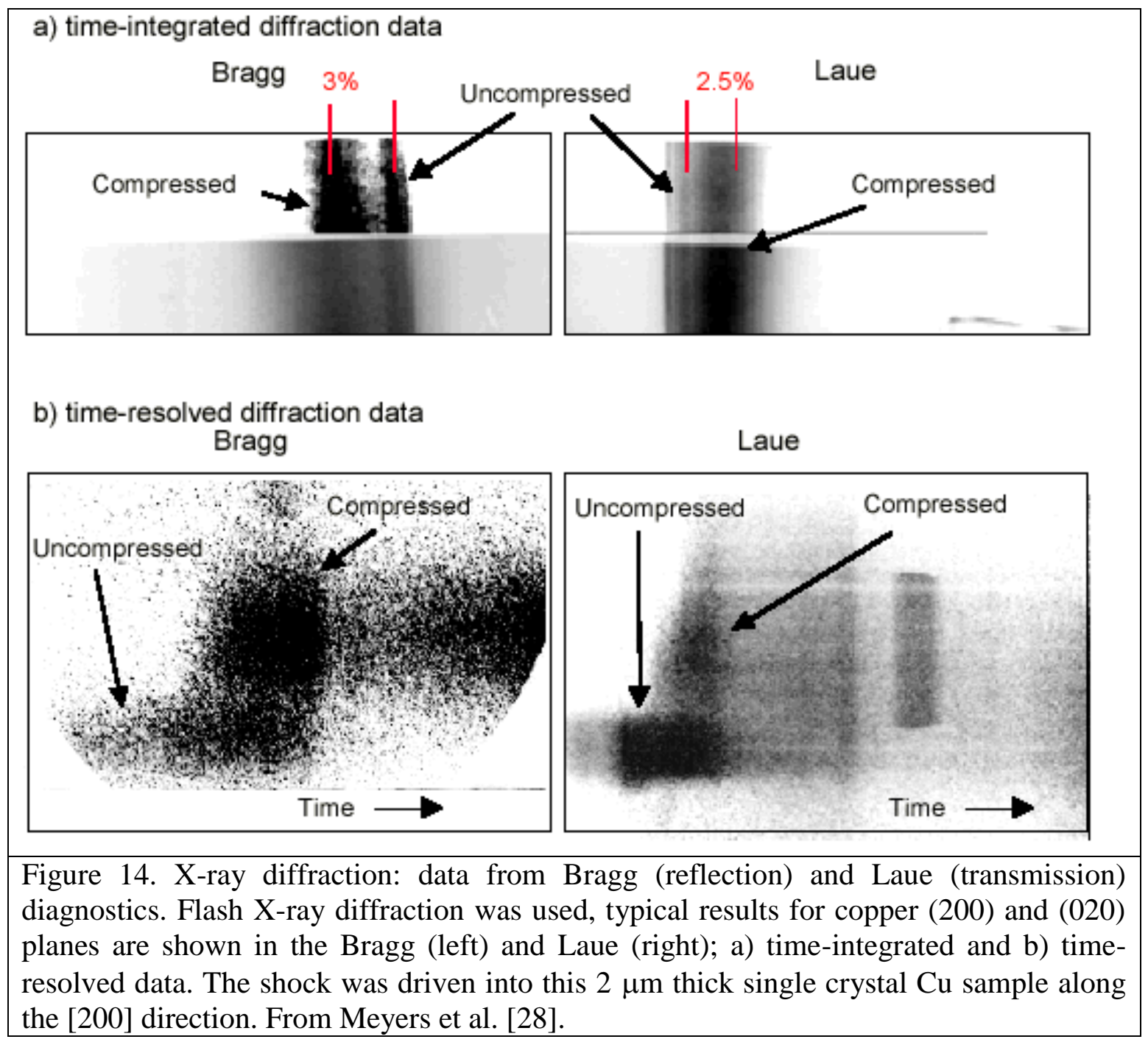




\section{Copper Shear Strength}

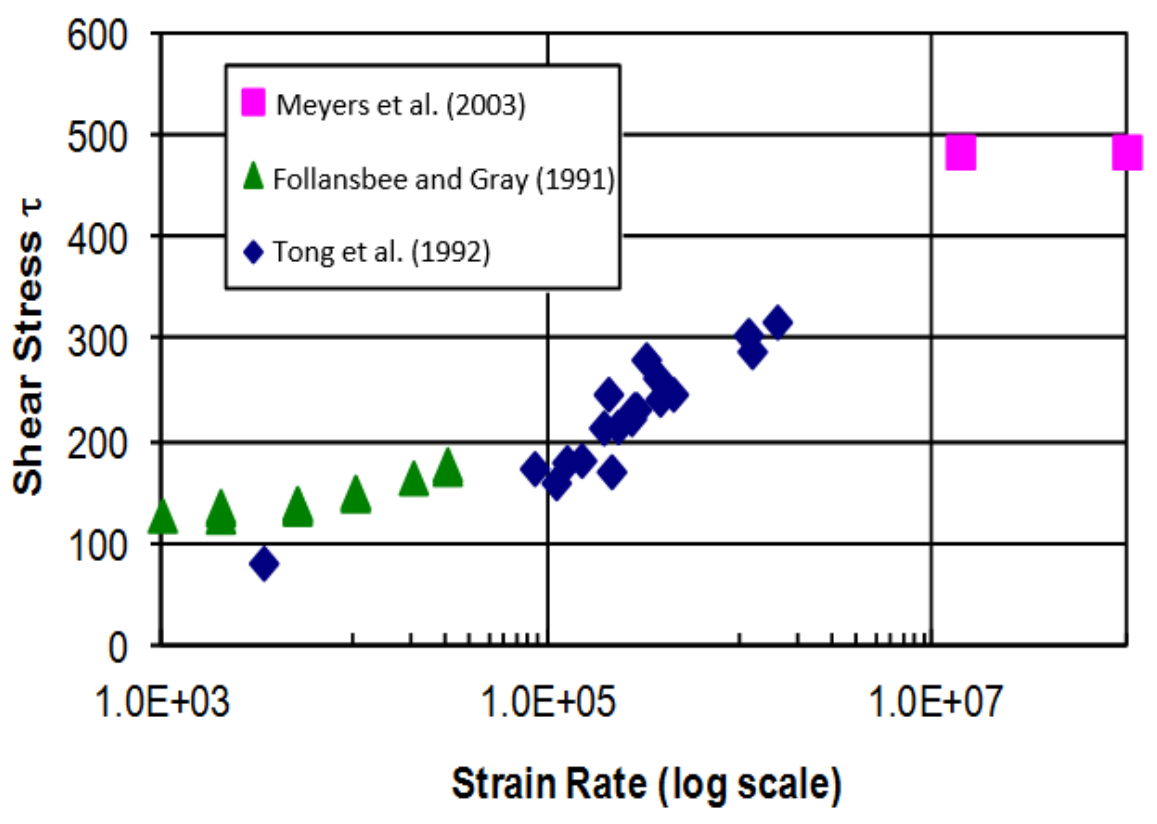

Figure 15. Copper shear strength results from the diffraction data from shock compression shown in Fig. 14. Adapted from Meyers et al. [28] 


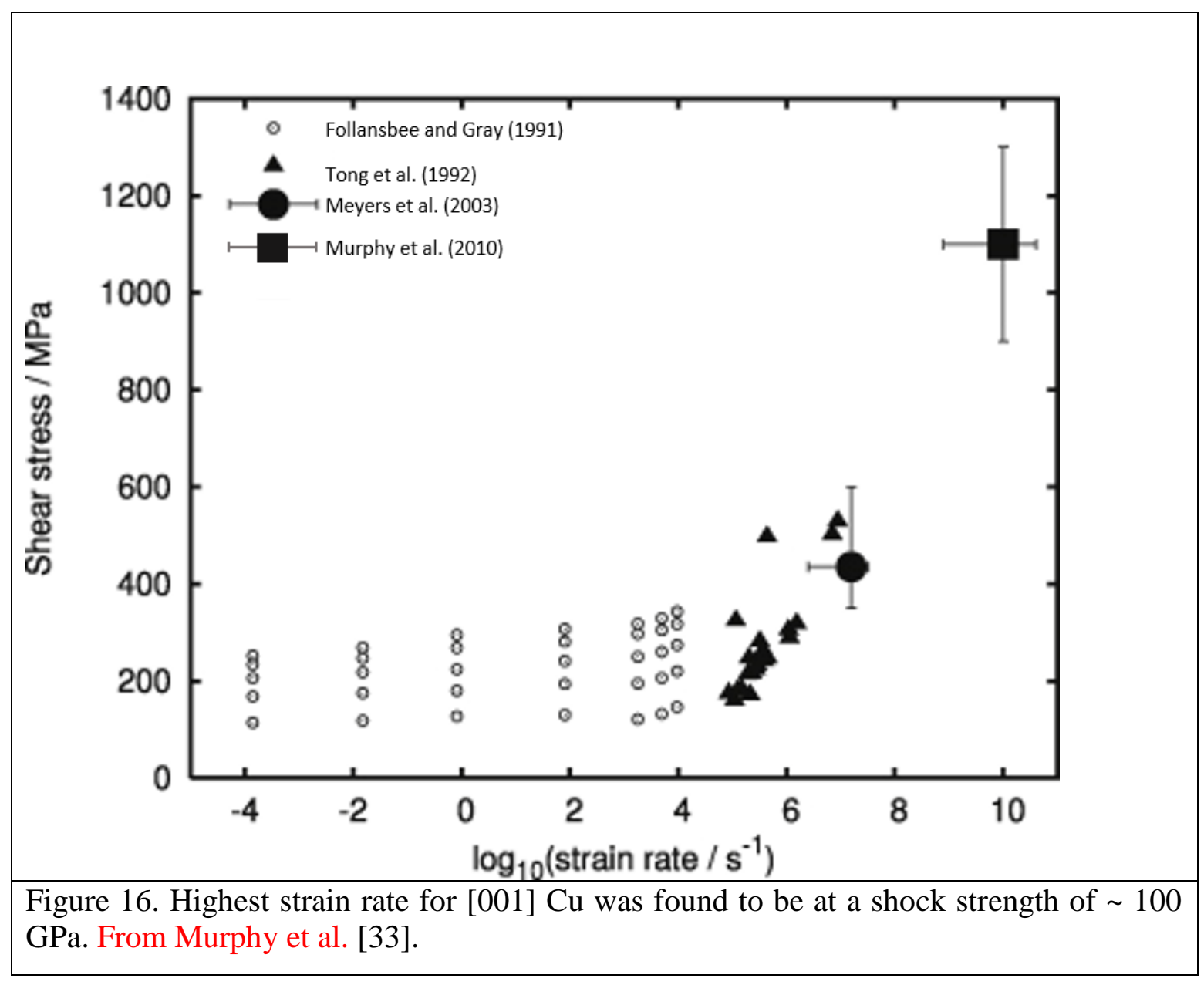




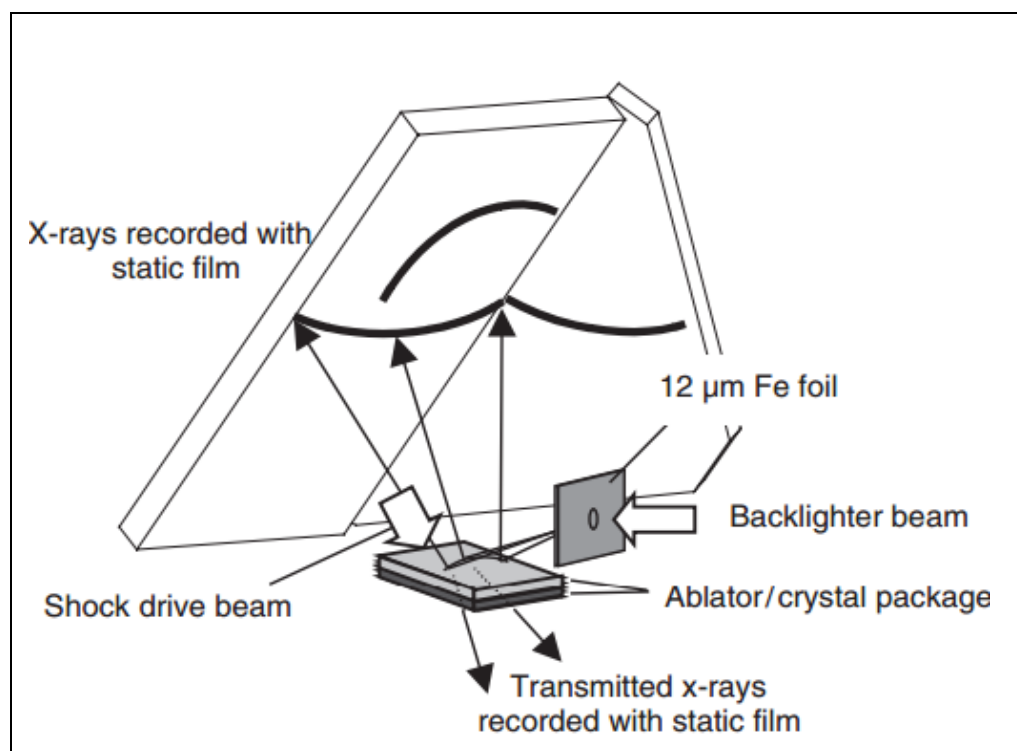

(a)

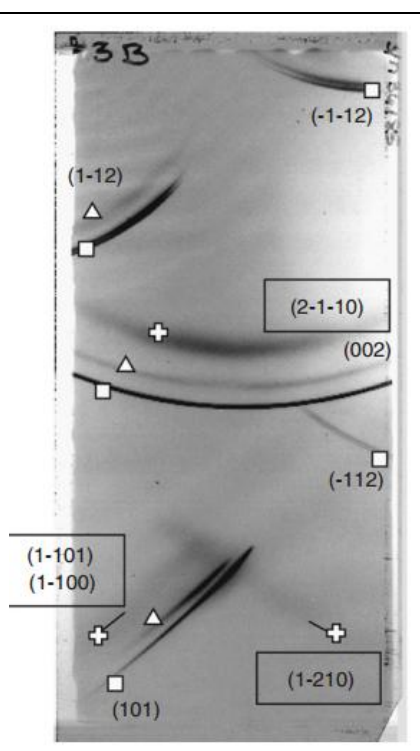

(b)

Figure 17. (a) Schematics of dynamic X ray diffraction setup showing simultaneous shock pulse propagation and diffraction of $\mathrm{X}$ rays and providing both time resolved and time integrated change in the diffraction angles due to changes in lattice parameter and/or appearance of a new phase; (b) Intersection of diffraction cones with recording plane for iron shocked at a pressure of $26 \mathrm{GPa}$, above the threshold for the alpha to epsilon transition $(=13 \mathrm{GPa})$. The diffraction lines from the static crystal are marked by squares, the ones from uniaxial compression by laser by triangles, and the lines from the epsilon phase are crosses. The shift in diffraction angle with pressure as well as the new lines provide a wealth of information on the extreme state accessed by shock compression. From Kalantar et al. [34]. 


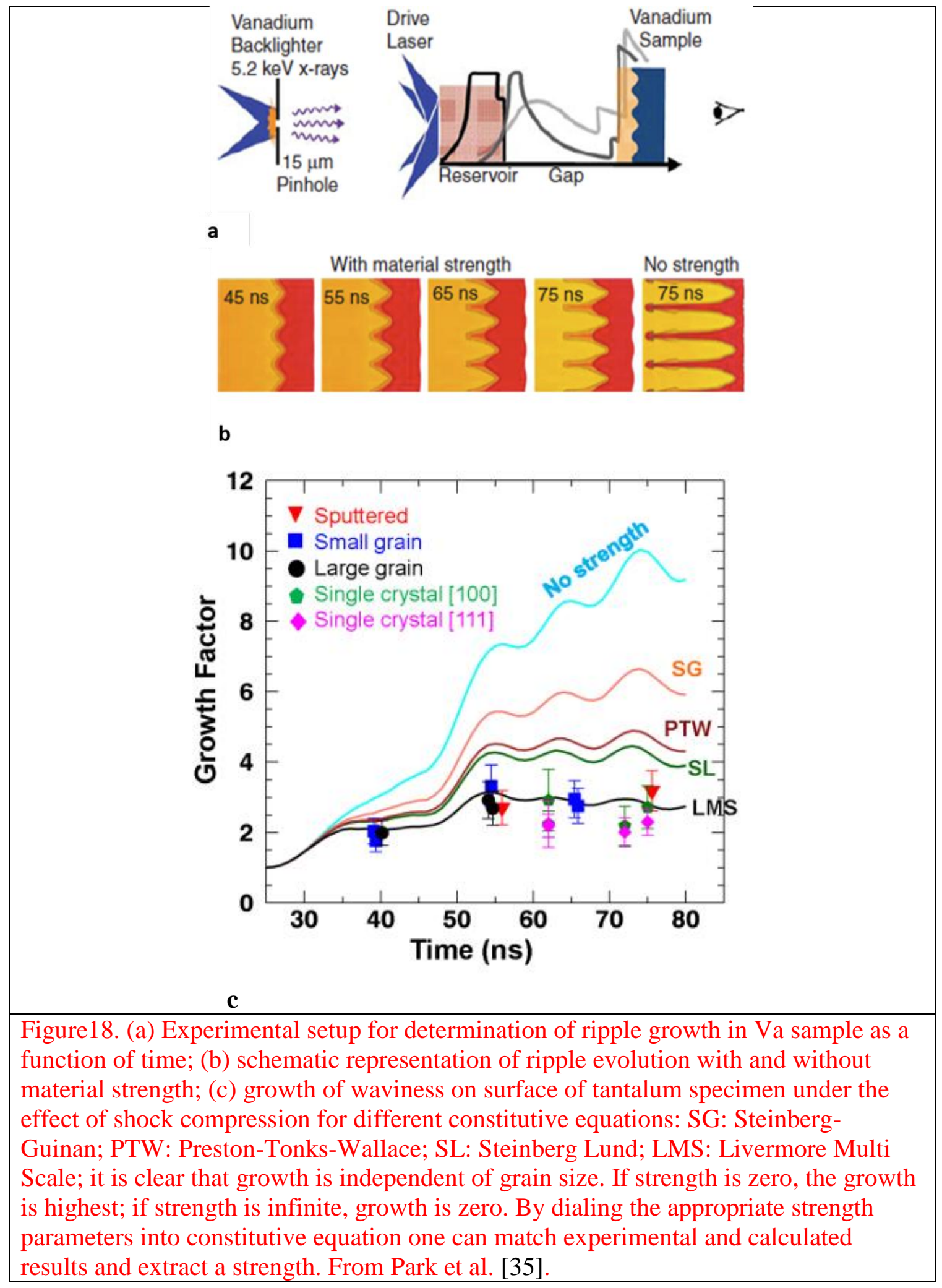




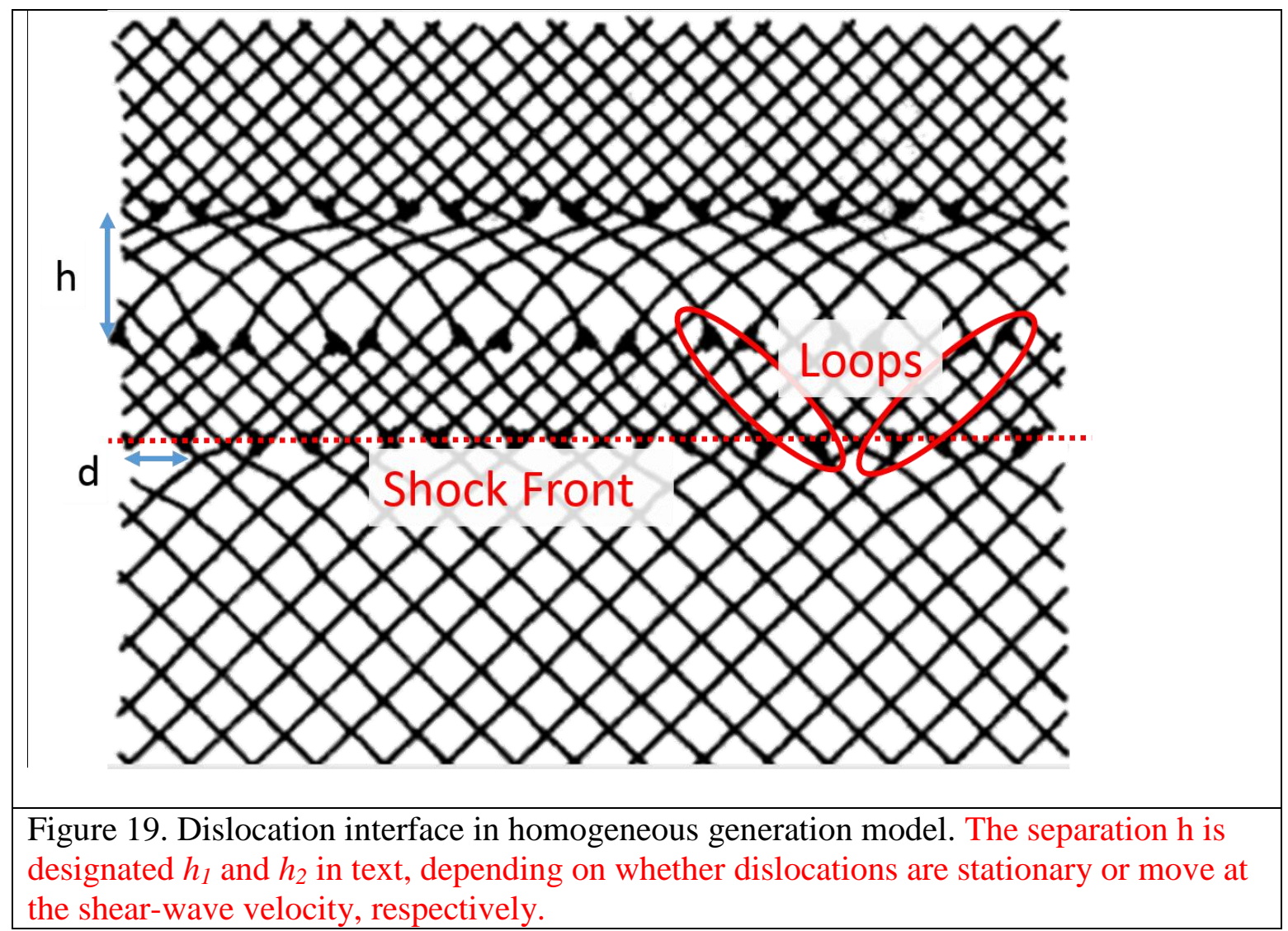




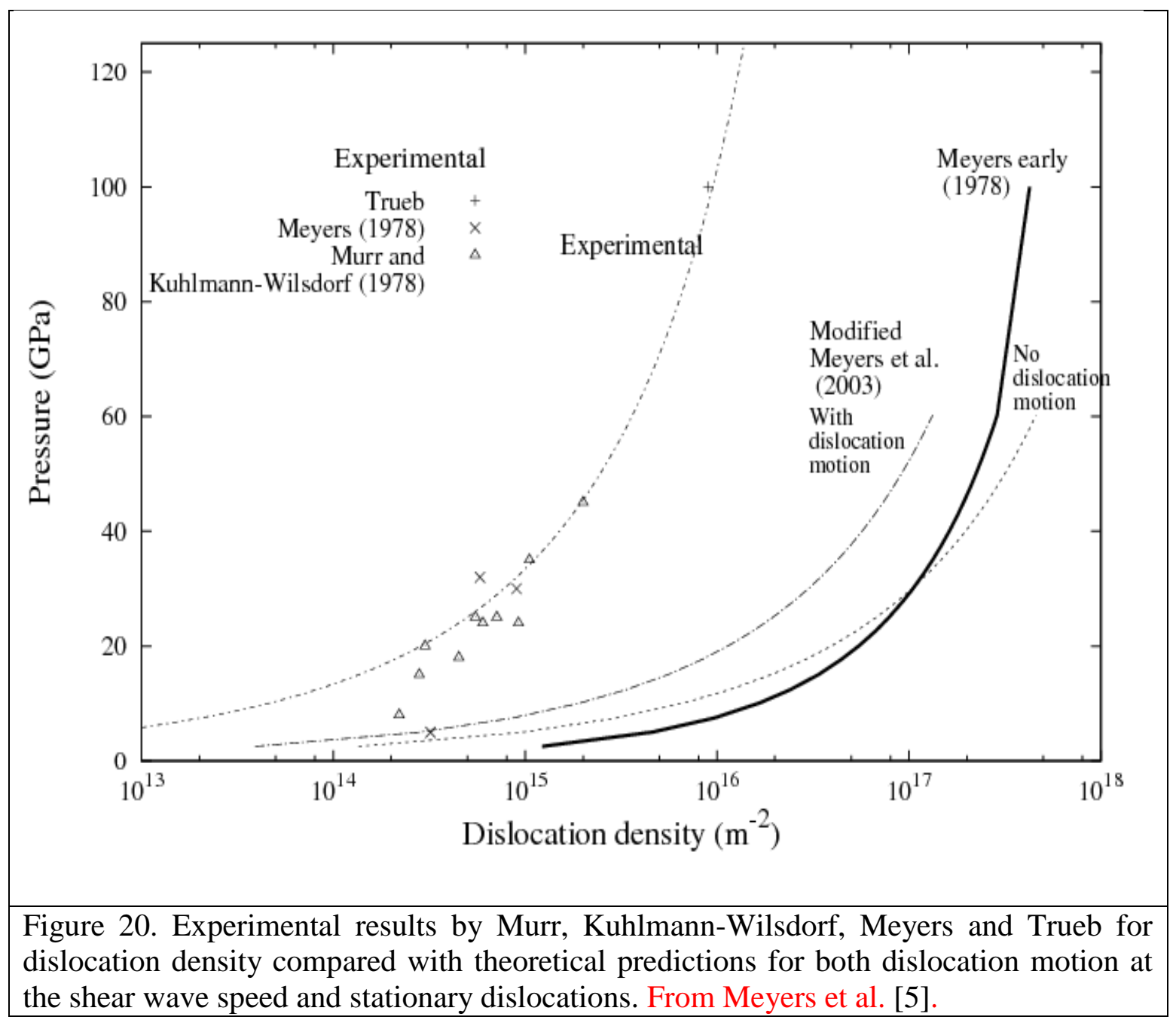




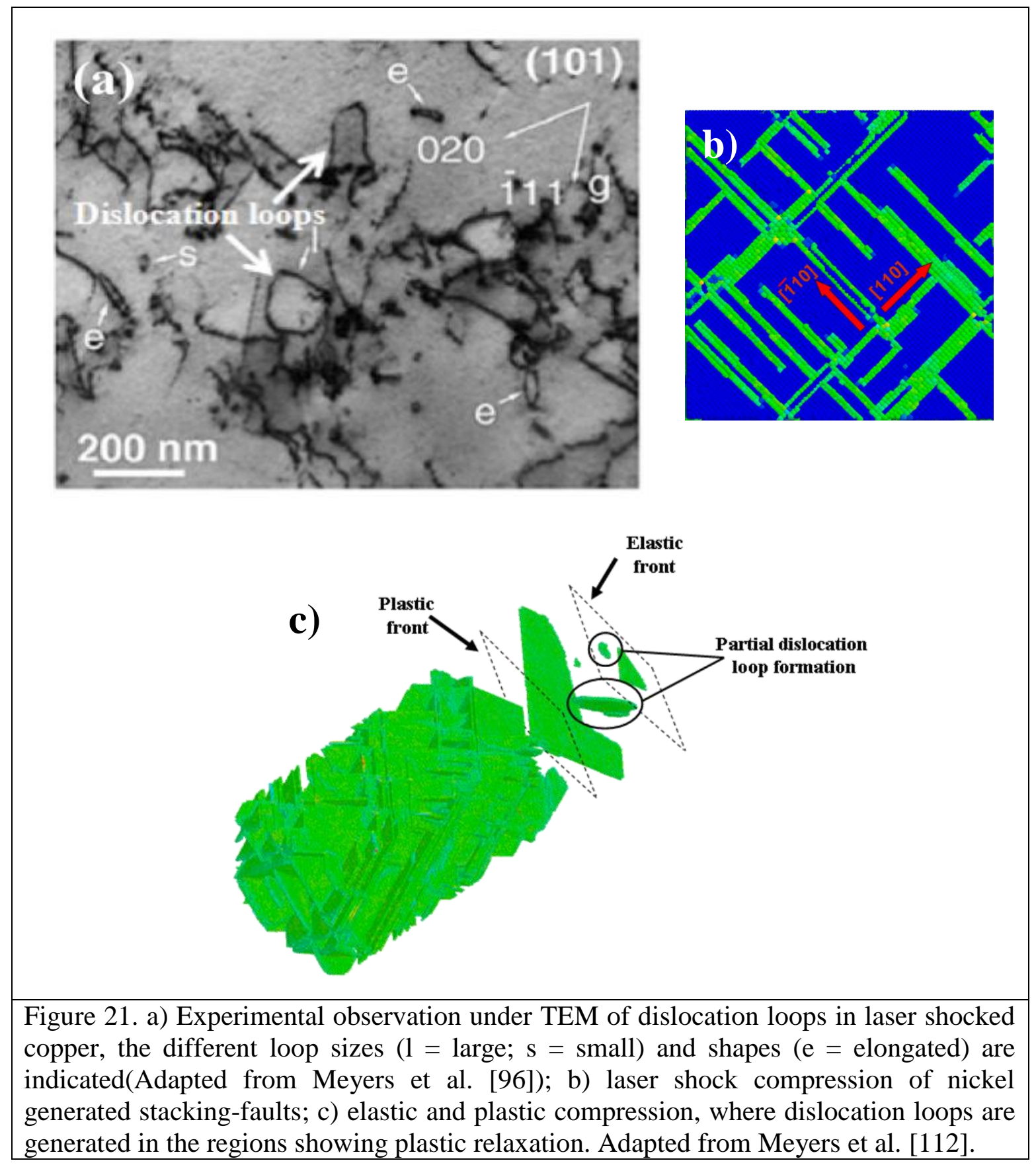


a)

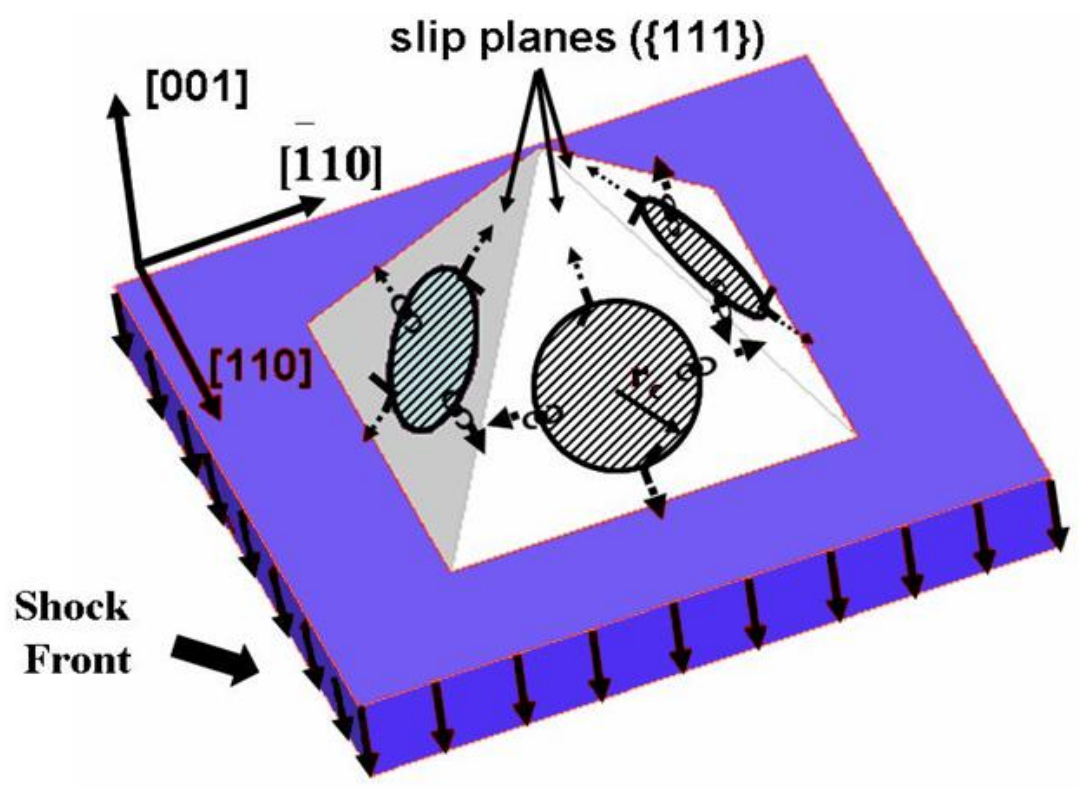

b)

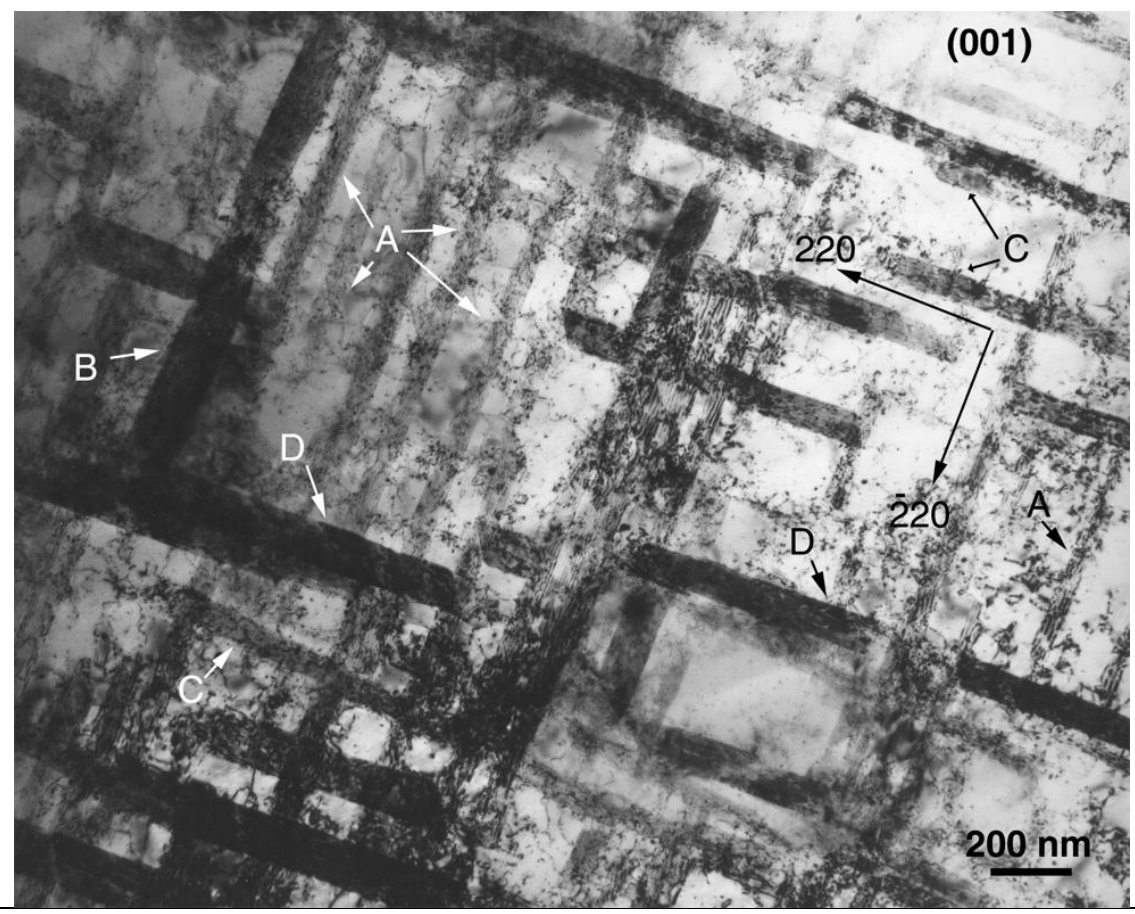

Figure 22. Face-centered cubic metals: generation of partial dislocation loops; a) Schematic showing dislocation loops nucleating at slip planes behind the shock front, shown in red with propagation along [001] Adapted from [5]; b) Stacking fault sets are marked as A, B, C, and D, where set A exhibits the highest density of occurrence (laser energy 205 J). From Schneider et al. [18] . 


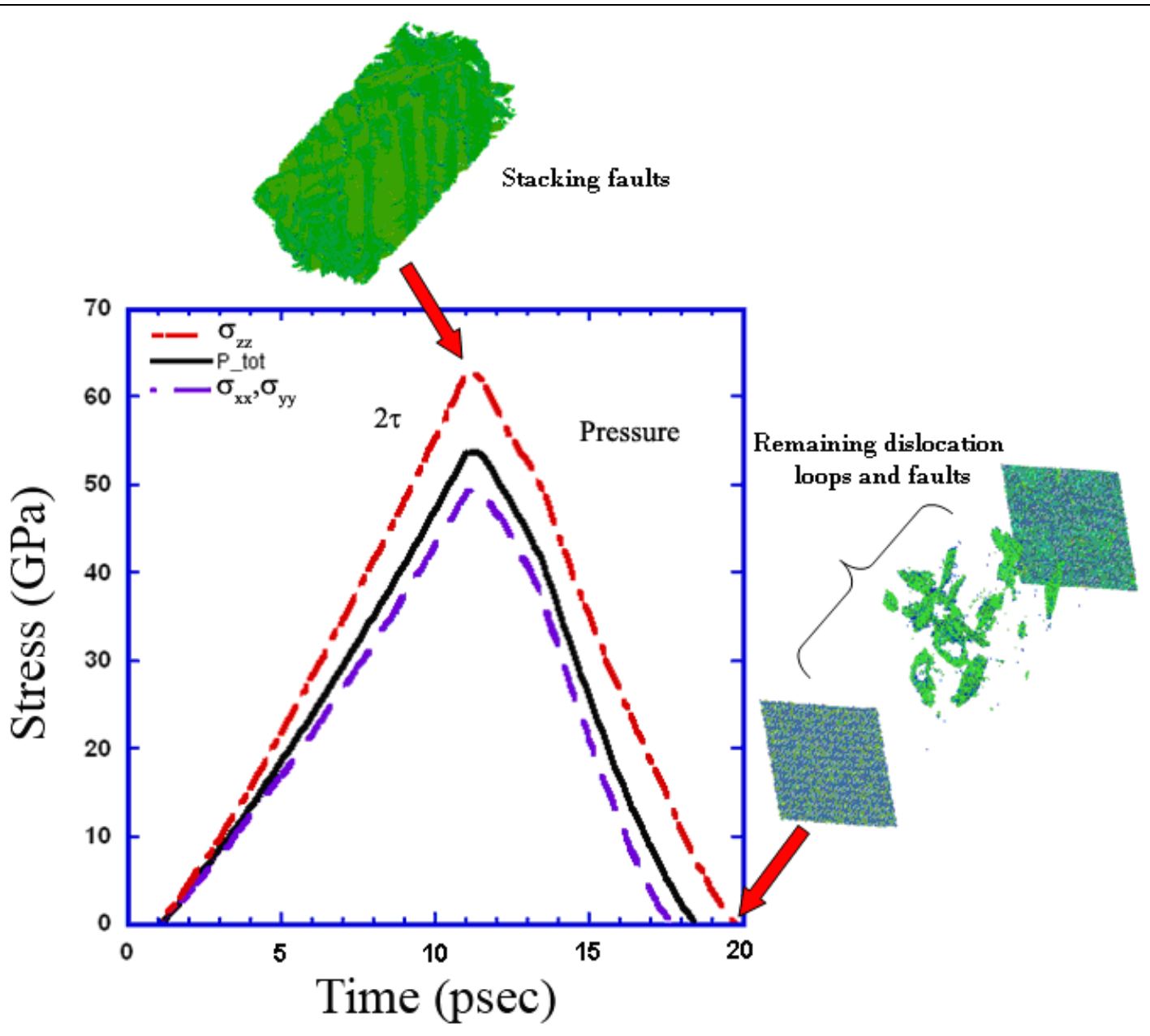

Figure 23. MD simulations showing peak applied stress $\left(\sigma_{z z}\right.$, equivalent to $\sigma_{11}$ in paper) during shock compression of nickel along [001], the lattice $\mathrm{z}$ direction. From Jarmakani et al.[44], Fig. 7. 


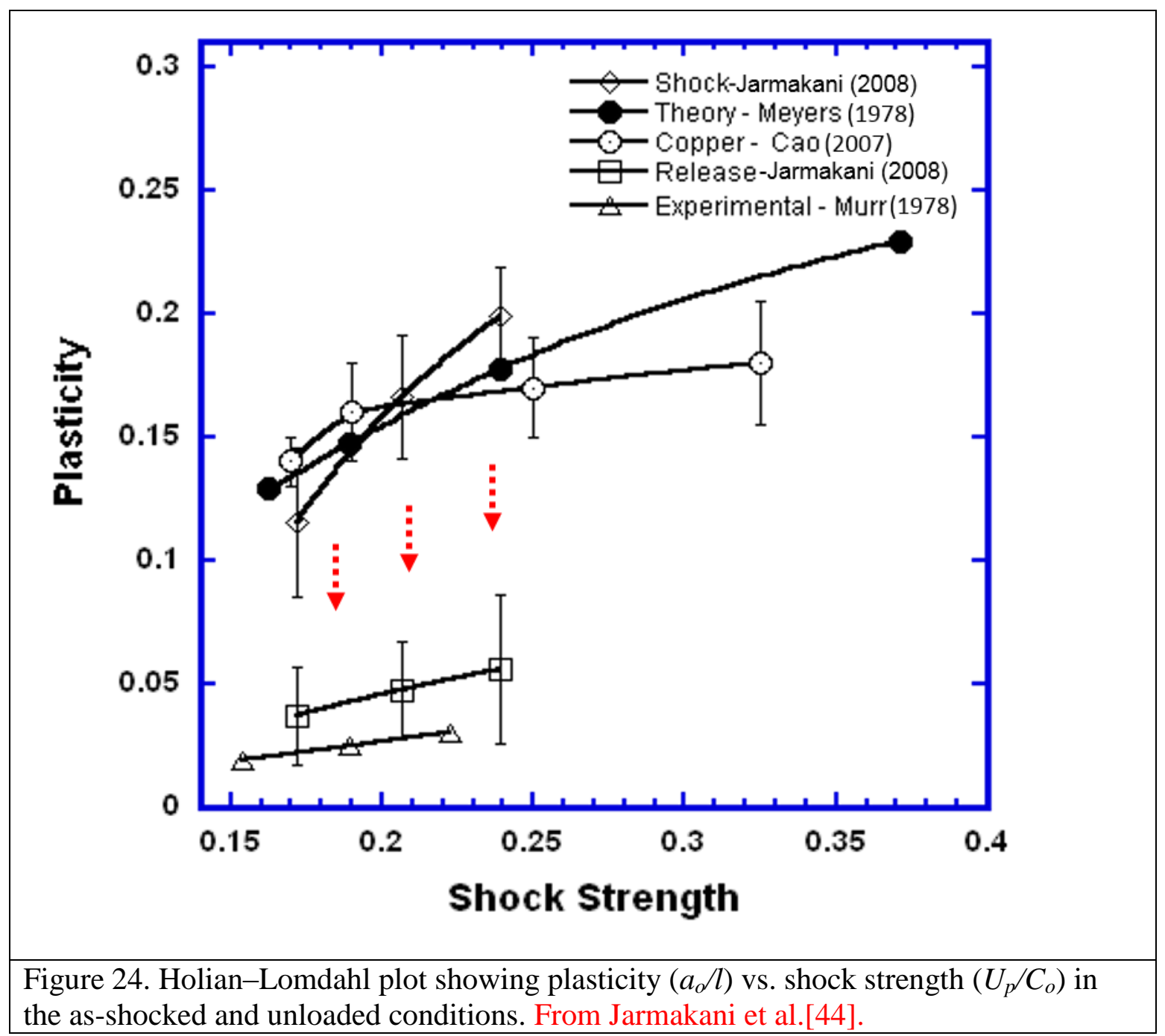




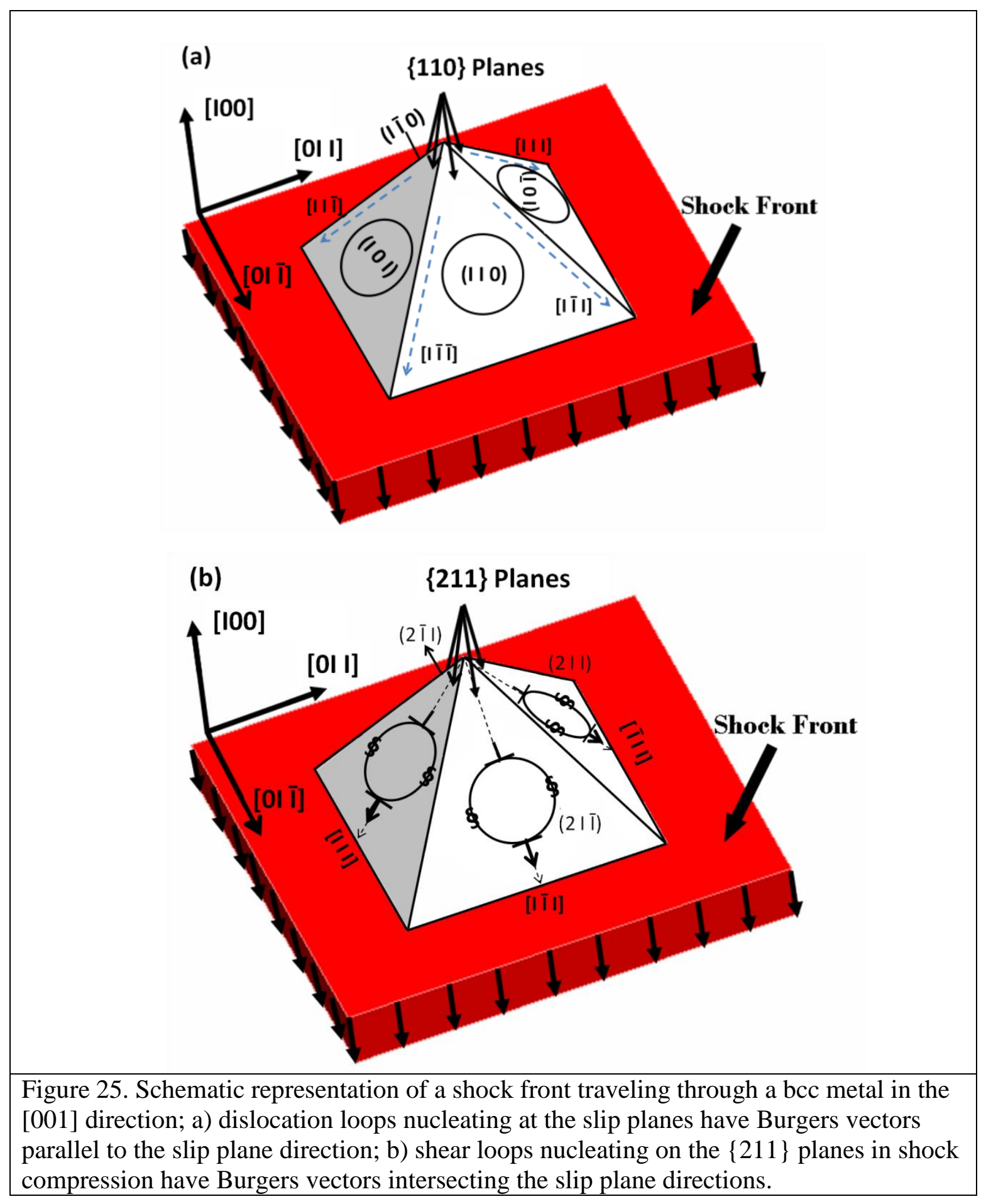




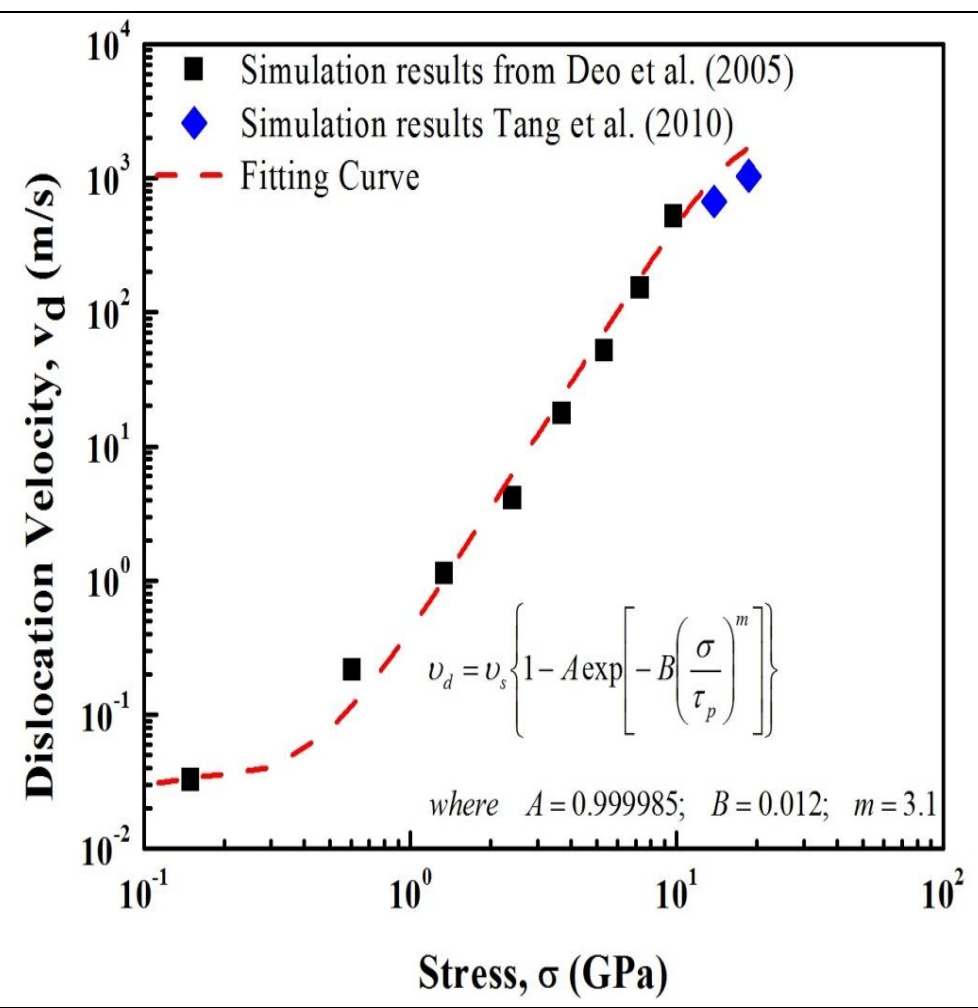

Figure 26. Predictions of dislocation velocity as a function of stress from the results of Tang et al. [49] and Deo et al. [50]. Adapted from Lu et al. [24]. 


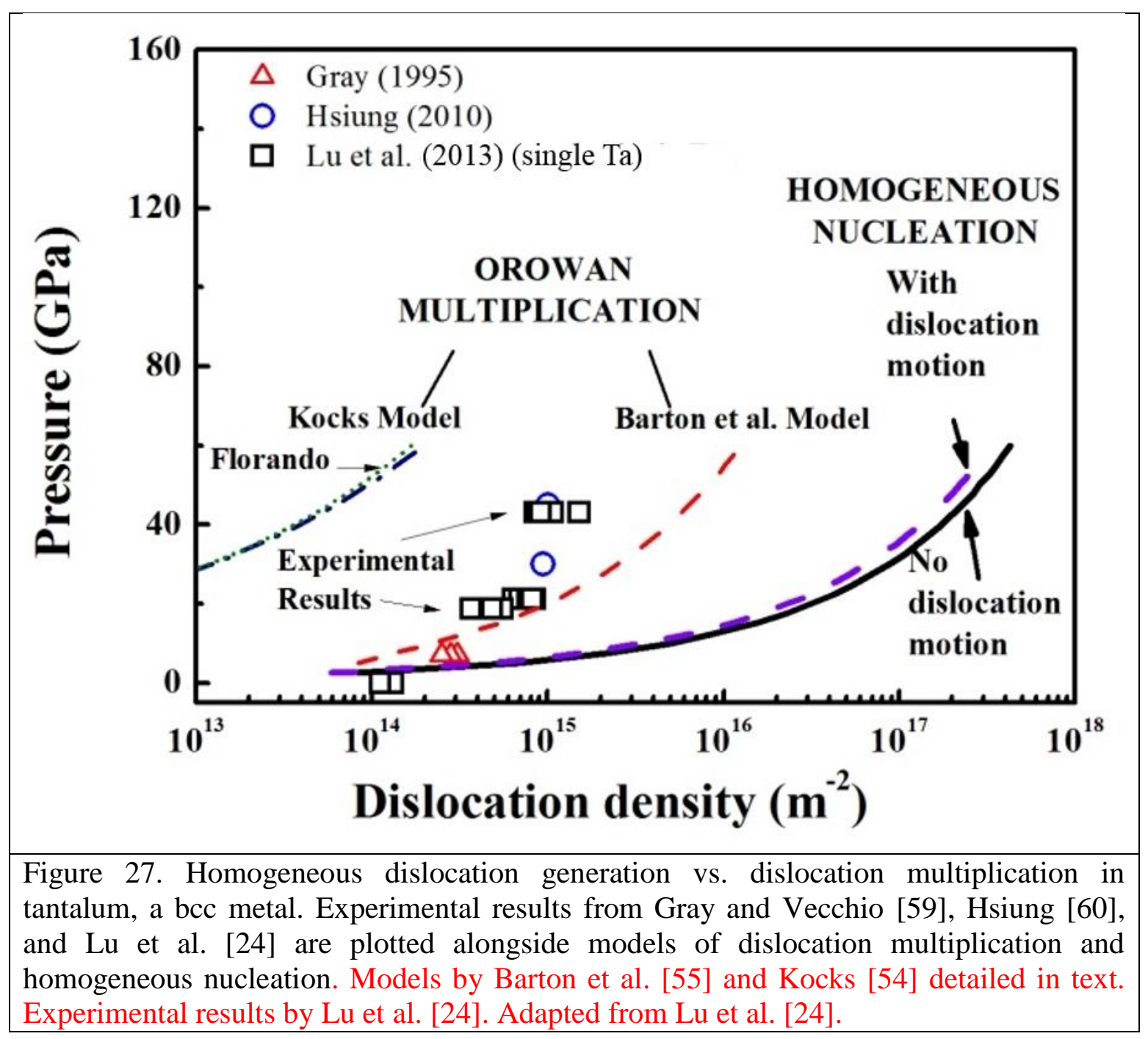




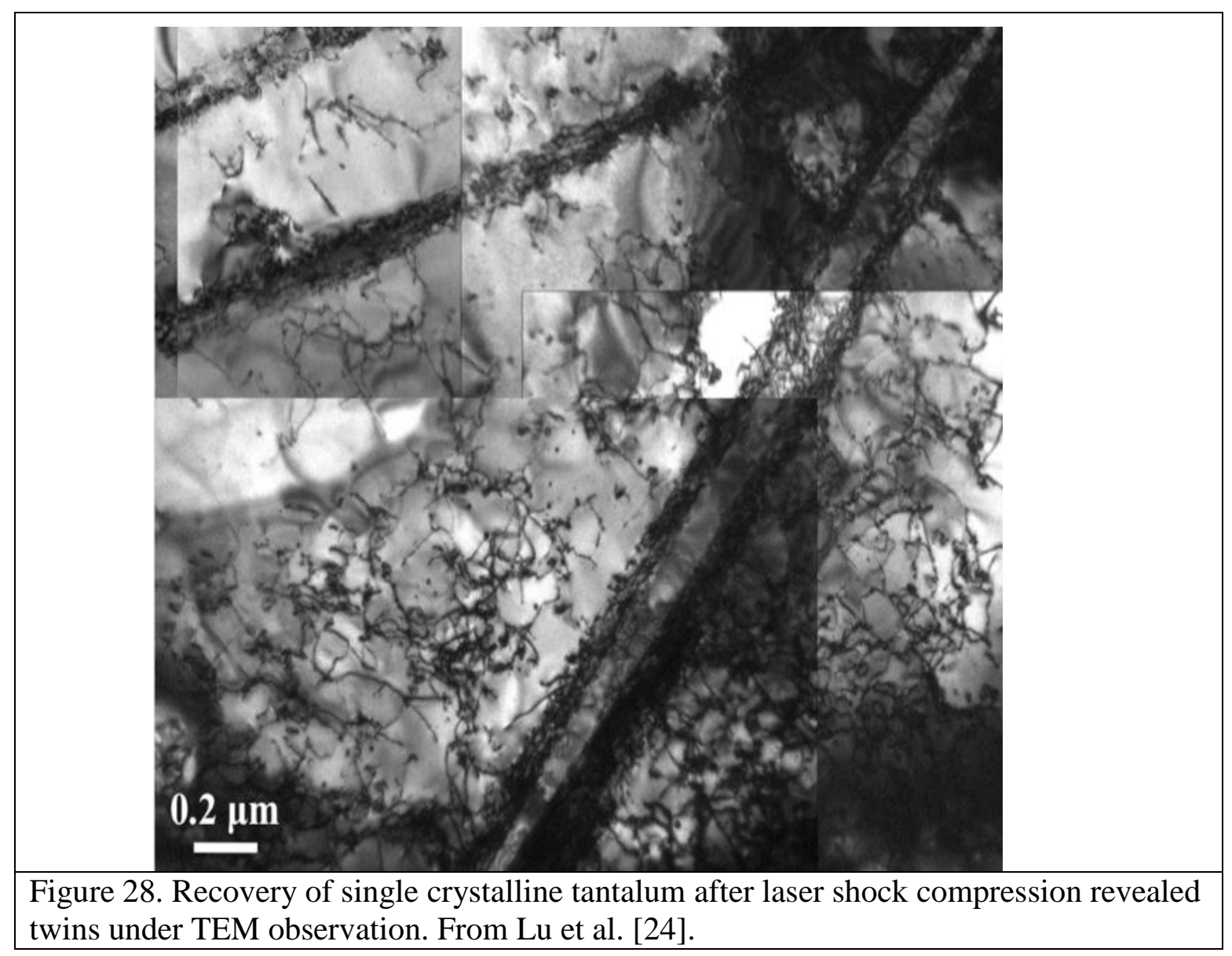




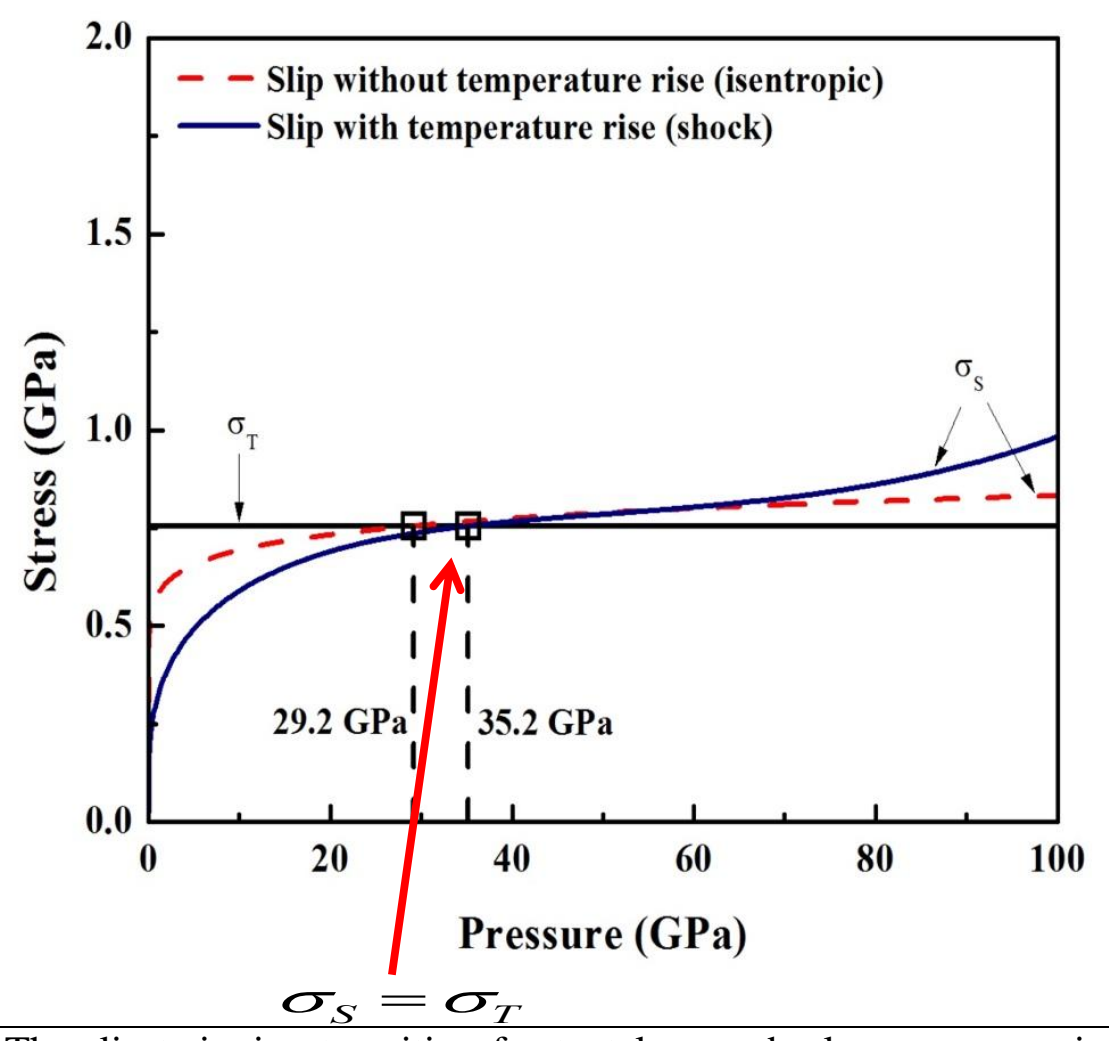

Figure 29. The slip-twinning transition for tantalum under laser compression is inferred to happen at a critical shock strength, above which slip cannot relieve the shock induced shear stress rapidly enough, and twinning commences (to relieve the shear stress). The twinning stress $\sigma_{\mathrm{T}}$ is assumed to be independent of temperature whereas the slip stress $\sigma_{\mathrm{S}}$ is dependent on it. From Lu et al. [24]. 


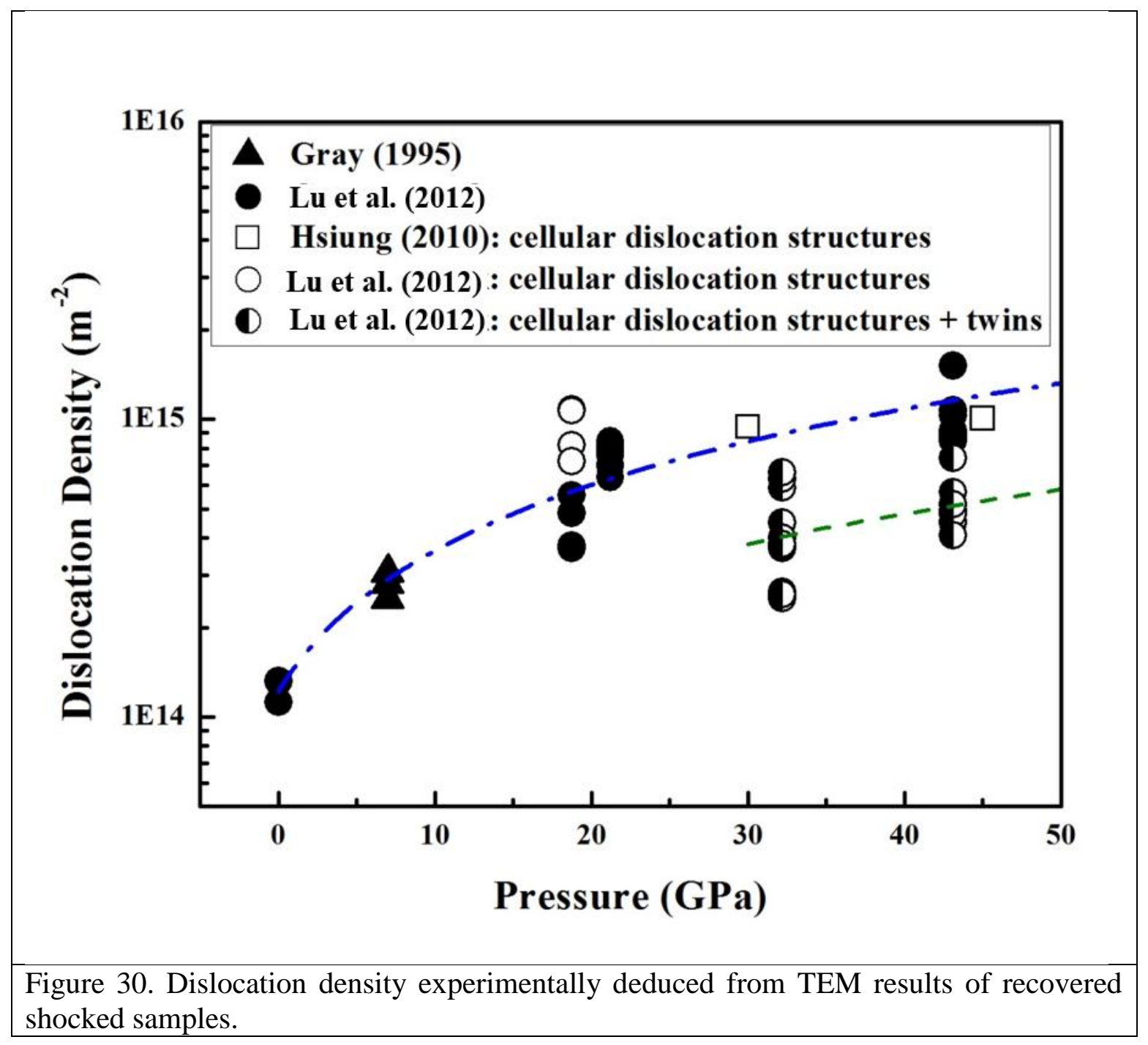




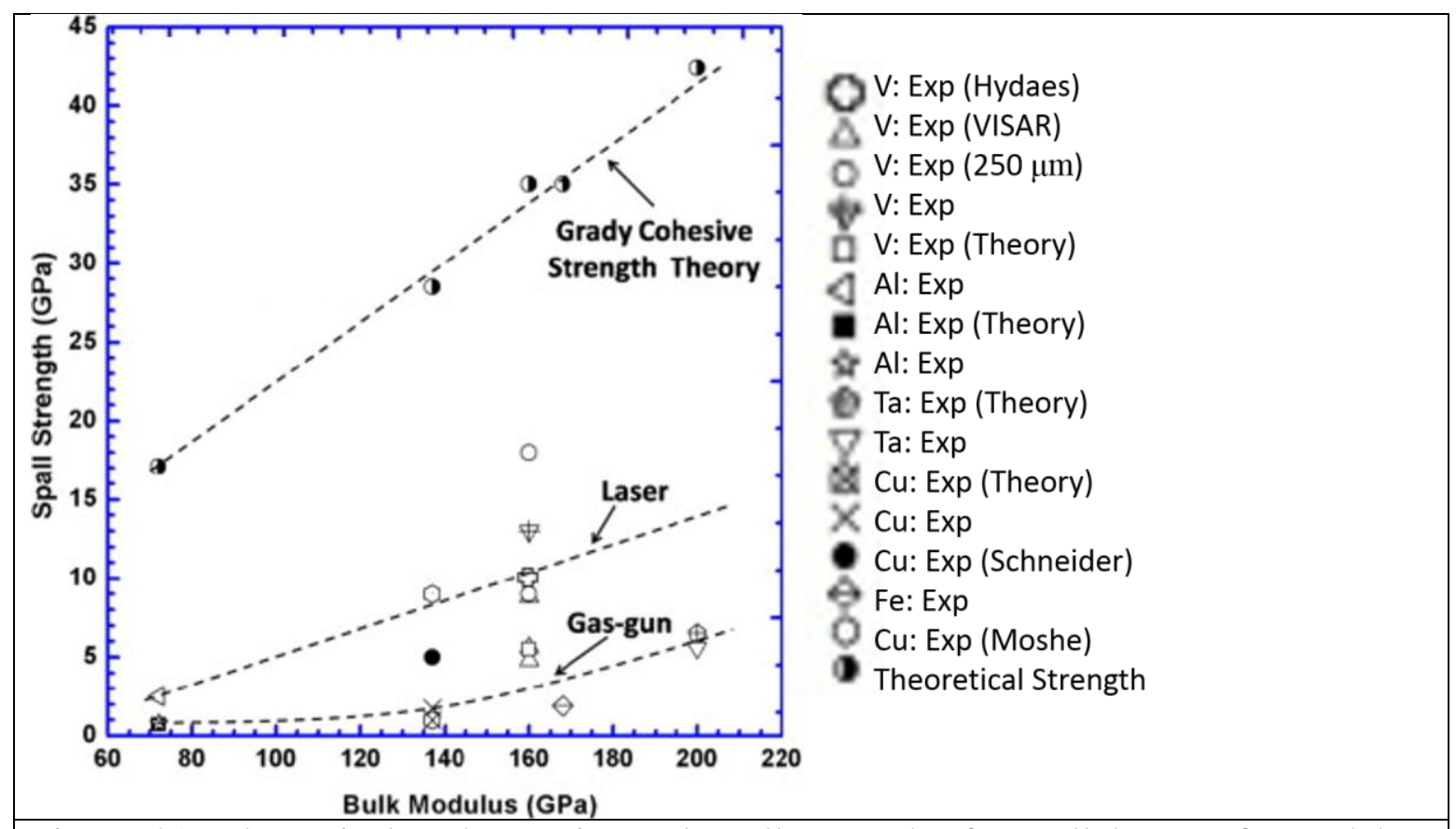

Figure 31. Theoretical and experimental spall strengths for well known fcc and bcc metals. Adapted from Jarmakani et al. [69]. 


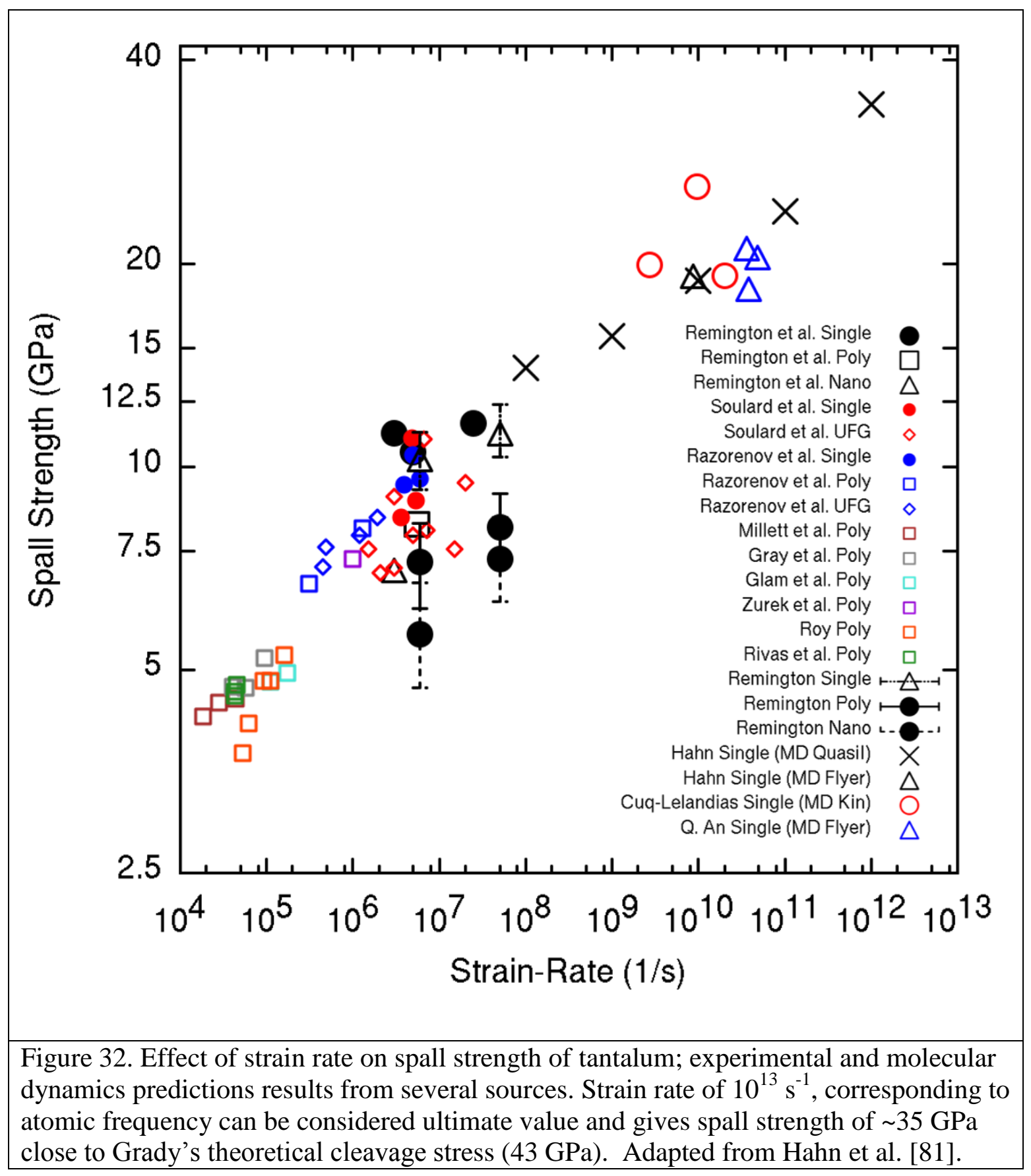




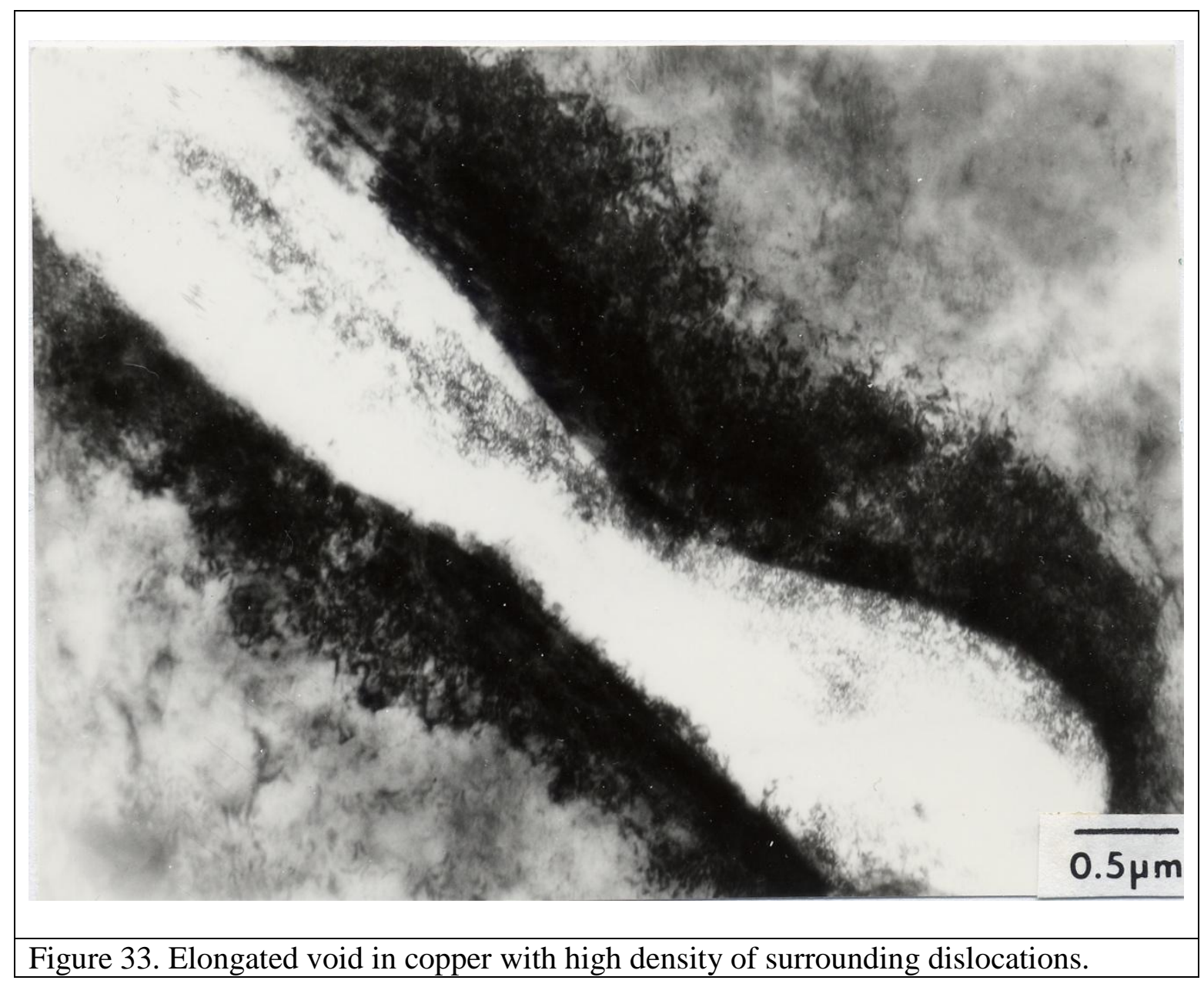




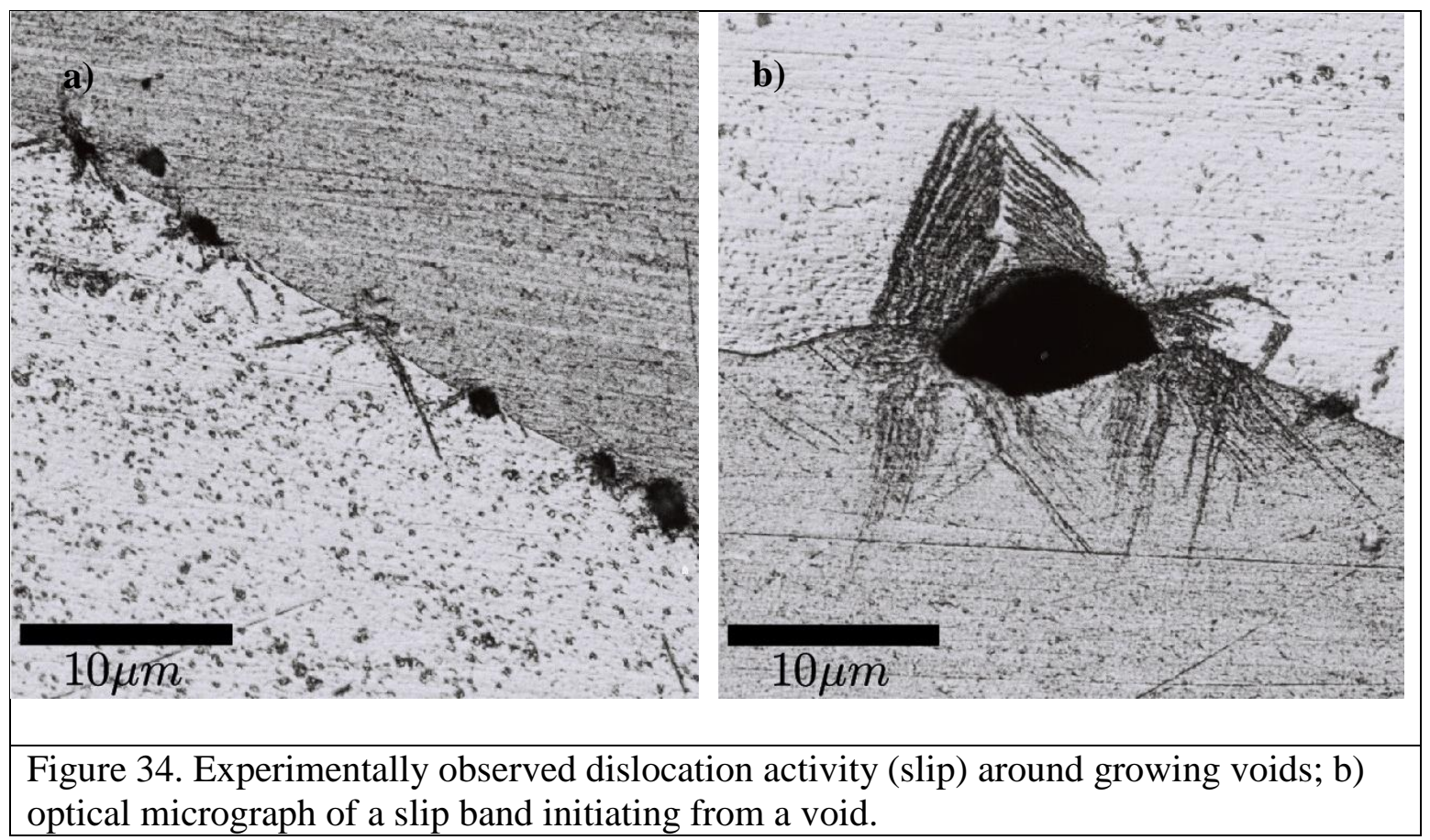




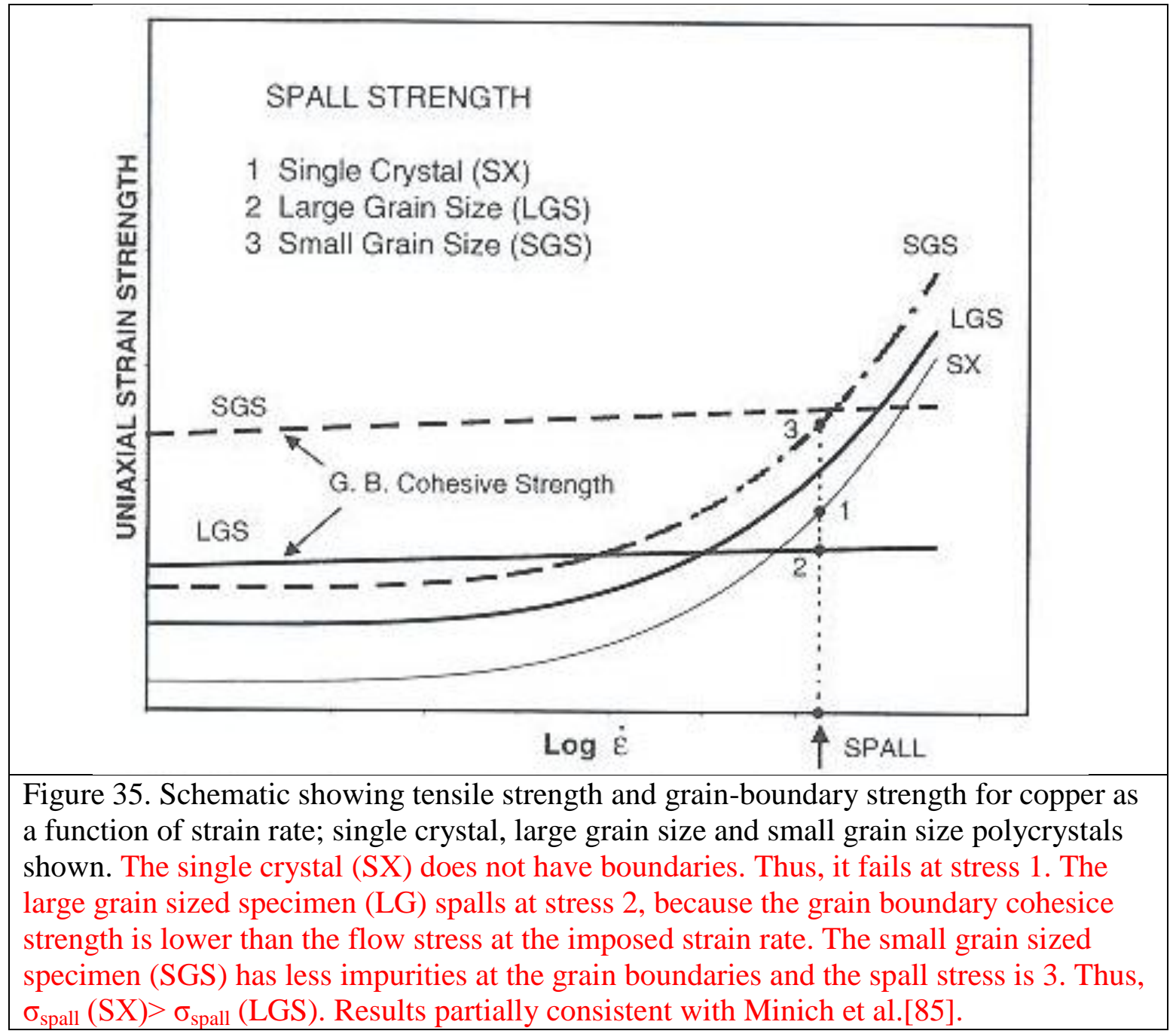




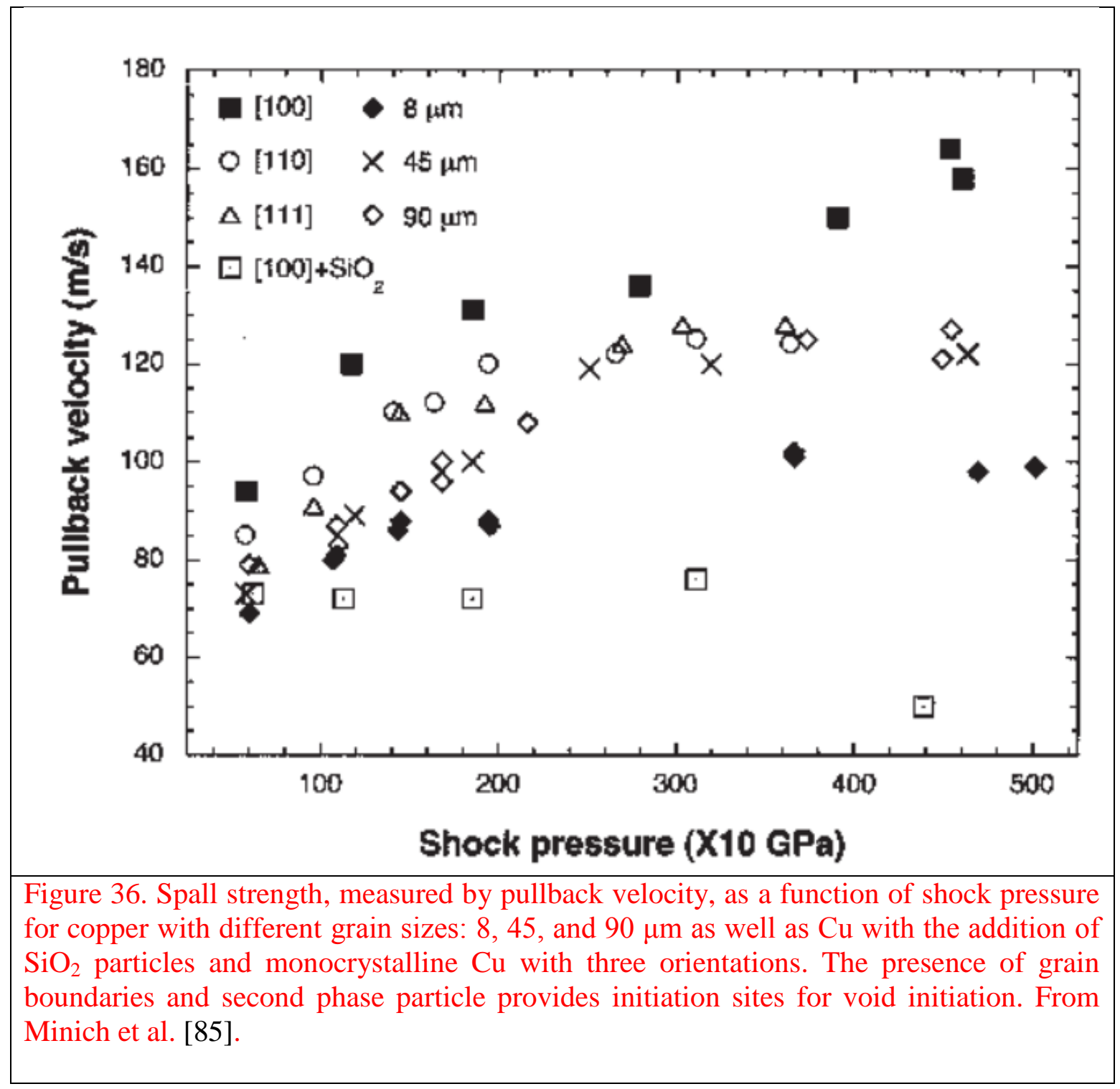




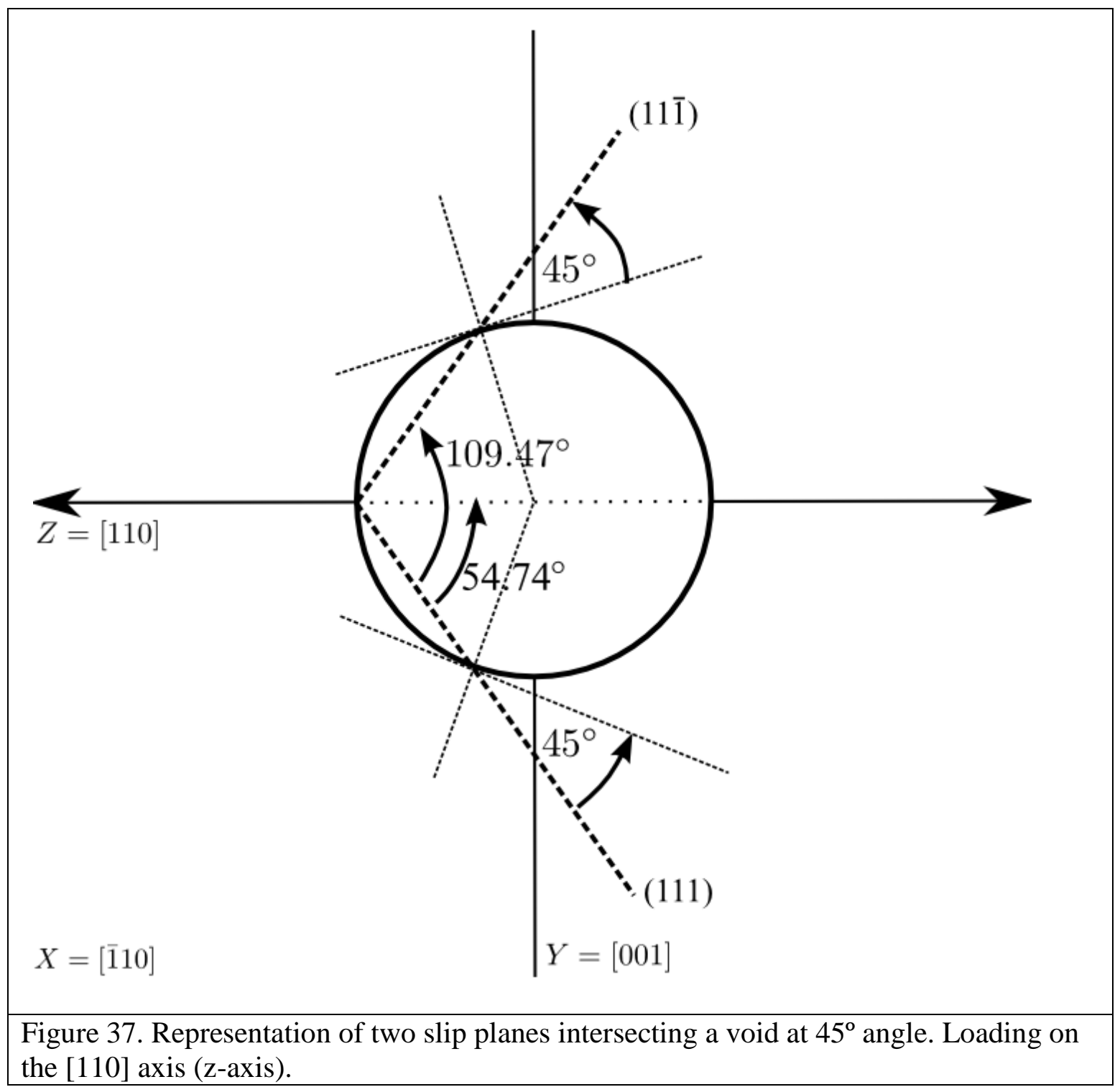




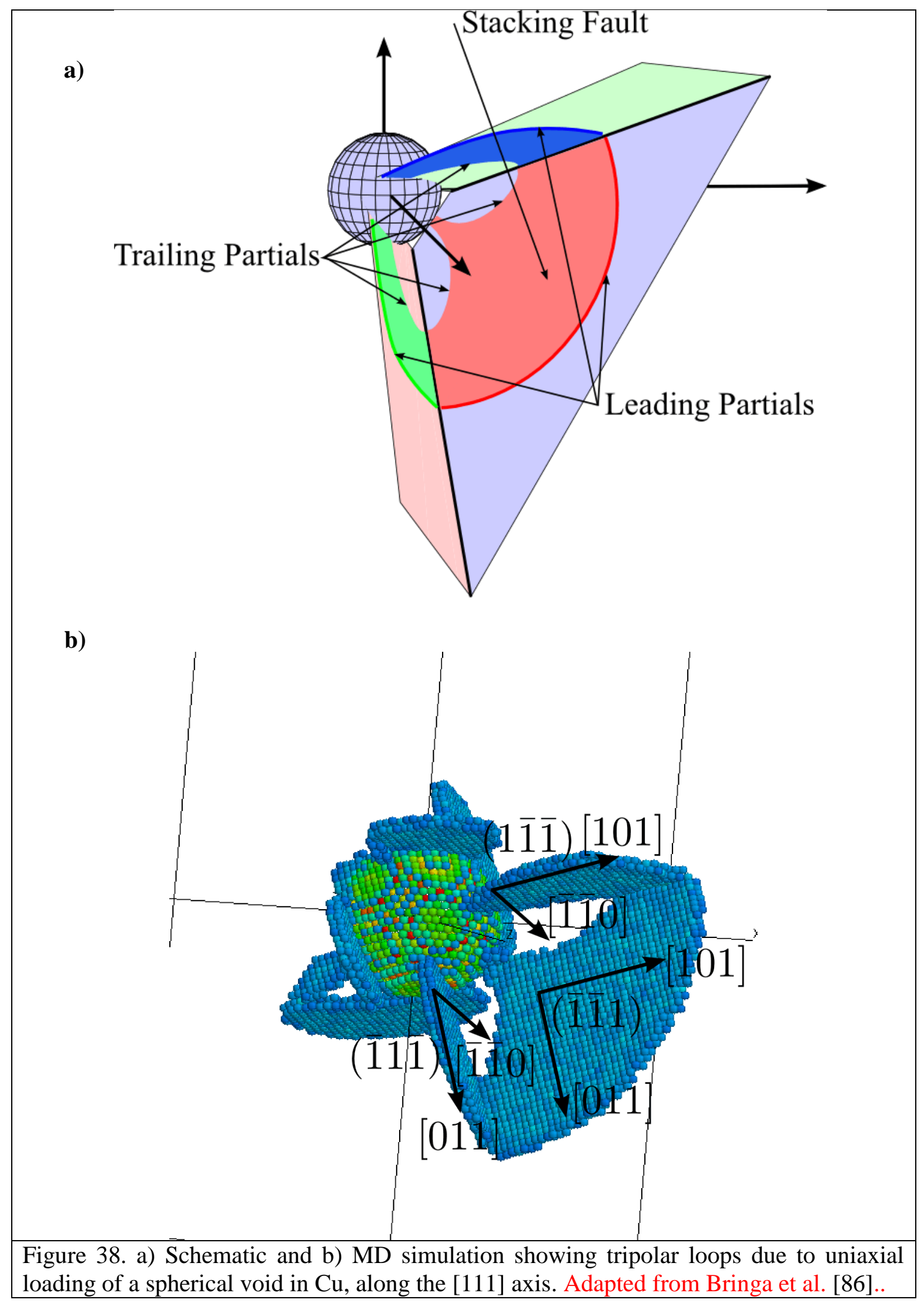




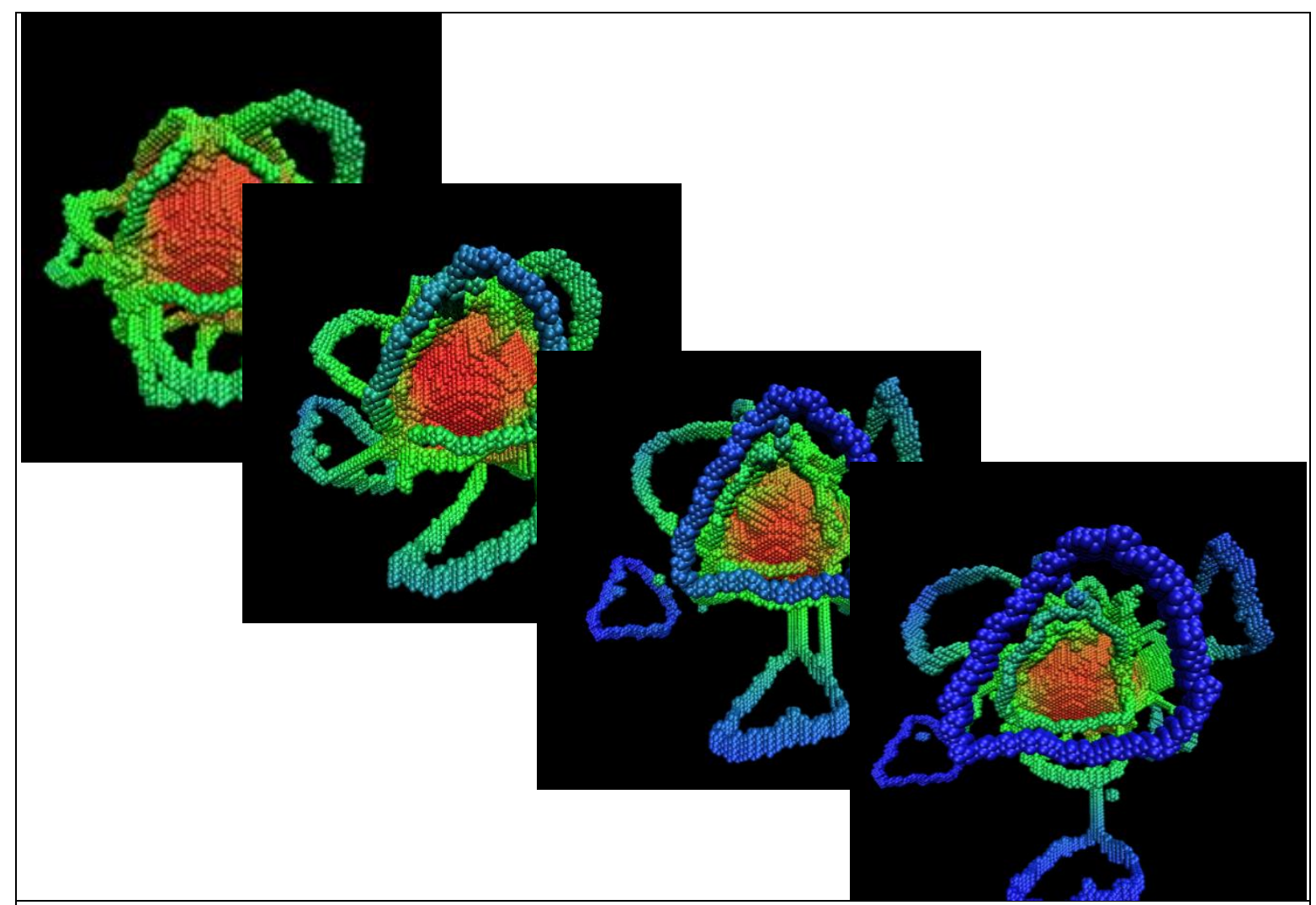

Figure 39. Molecular dynamics sequence showing shear and prismatic loop formation in single crystalline tantalum for high strain rates $\left(\sim 10^{8} \mathrm{~s}^{-1}\right)$ under hydrostatic tension.

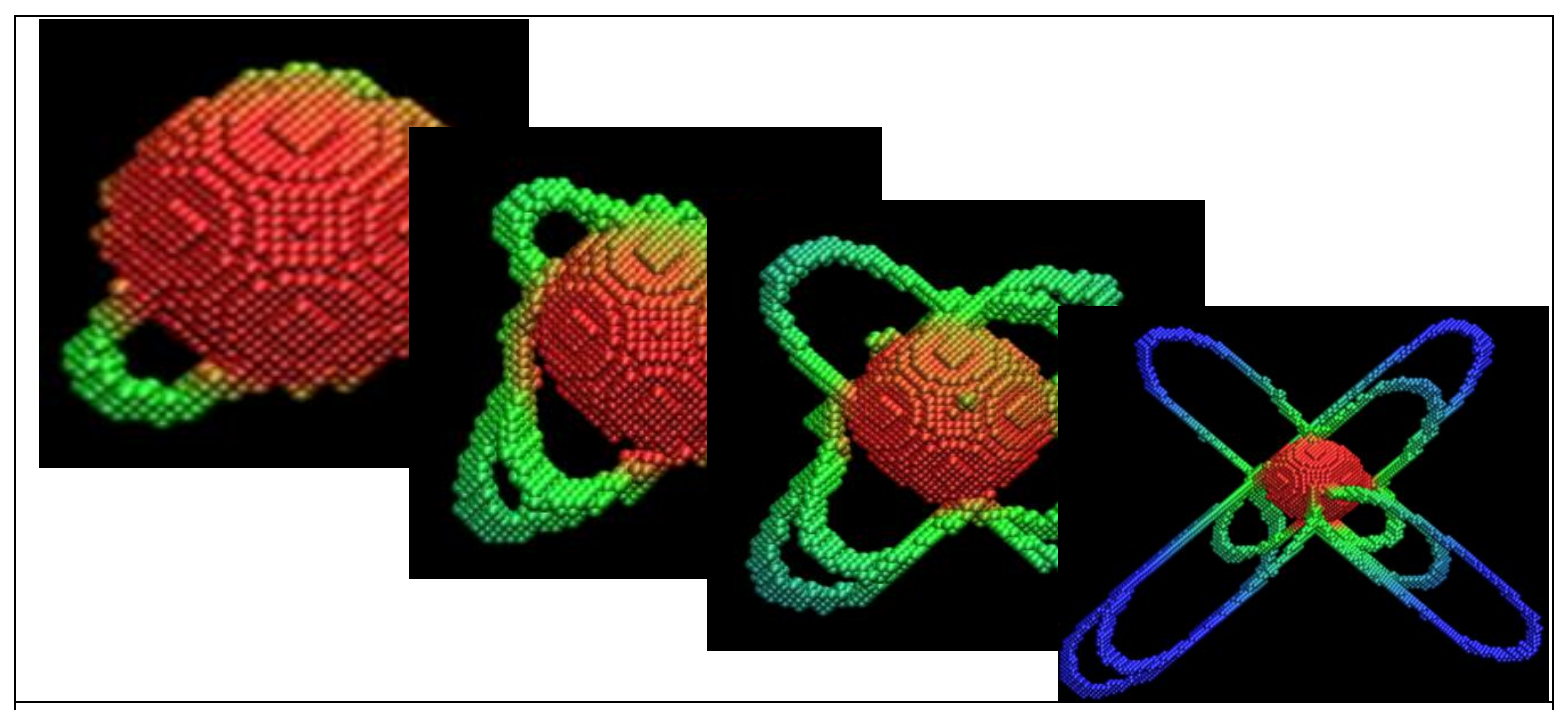

Figure 40. Molecular dynamics sequence showing shear loop formations in single crystalline tantalum for high strain rates $\left(\sim 10^{8} \mathrm{~s}^{-1}\right)$ under uniaxial compression. 
a)

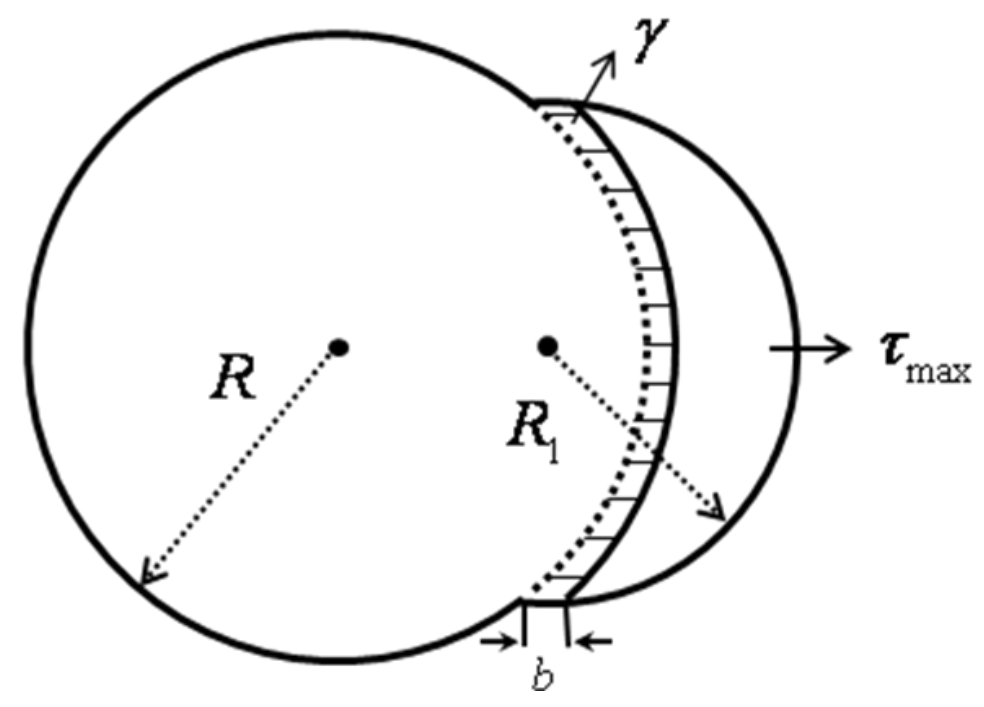

b)

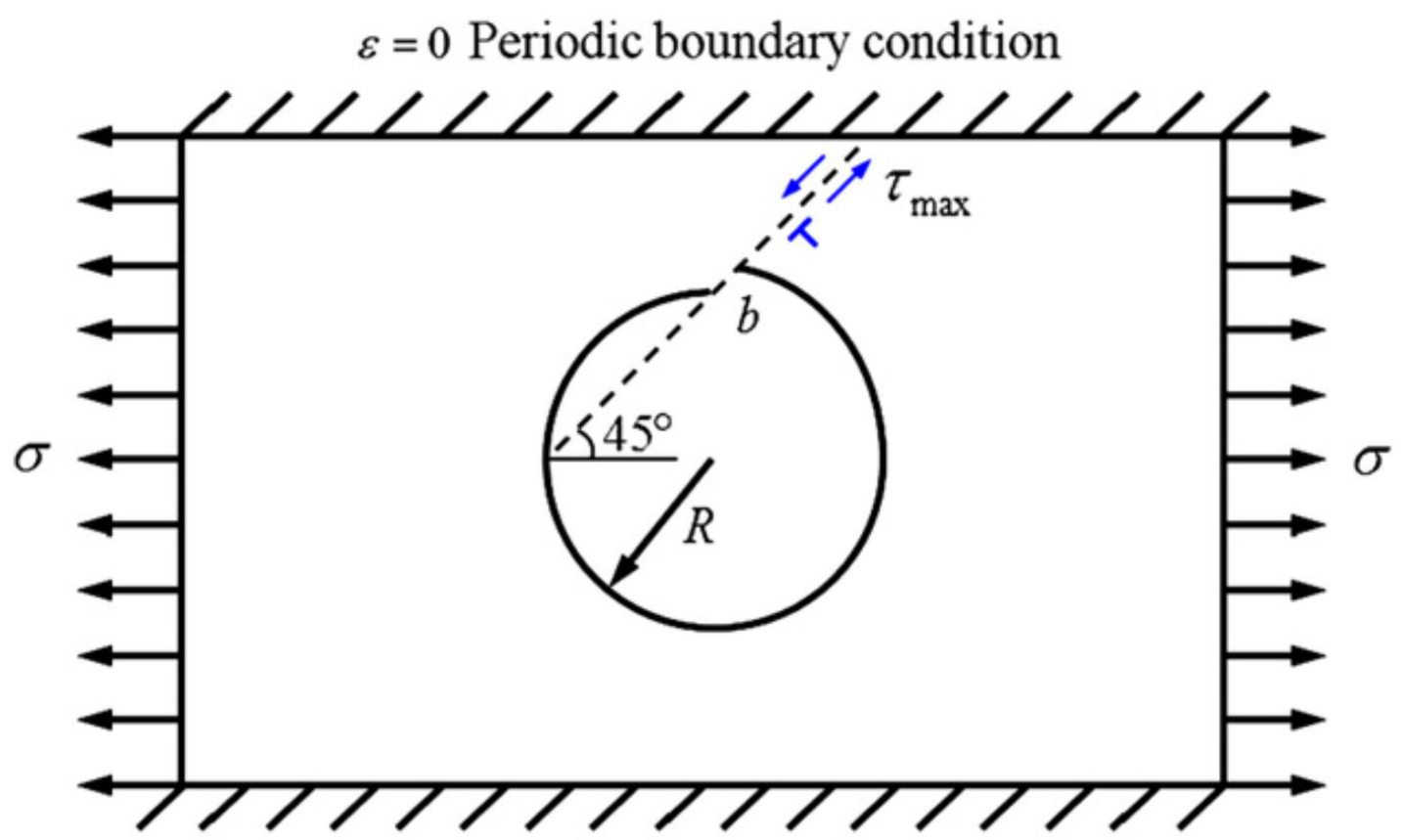

Figure 41. Representation of dislocation emission from a void for uniaxial tension: a) top view; and b) side view showing shear loop emission. 


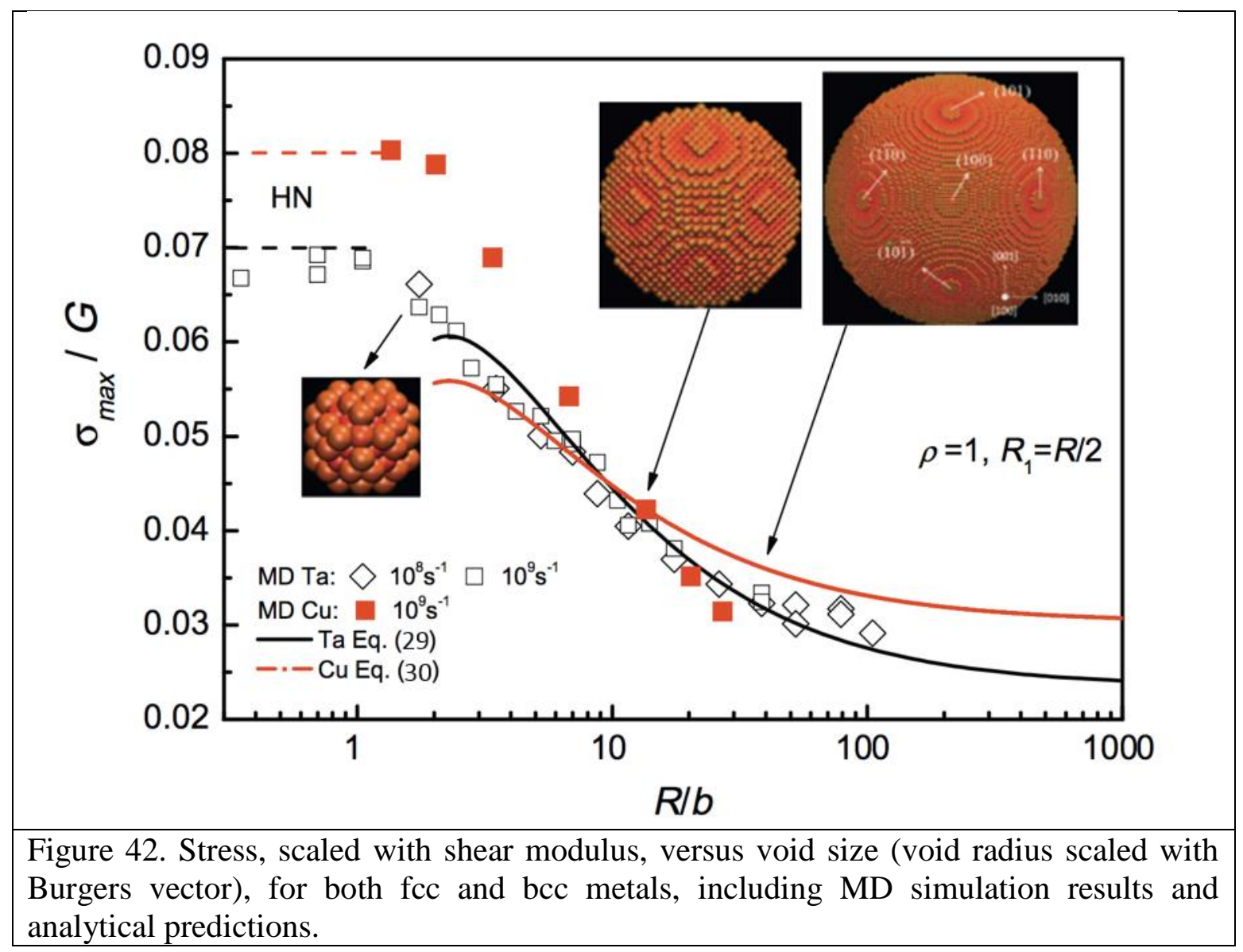




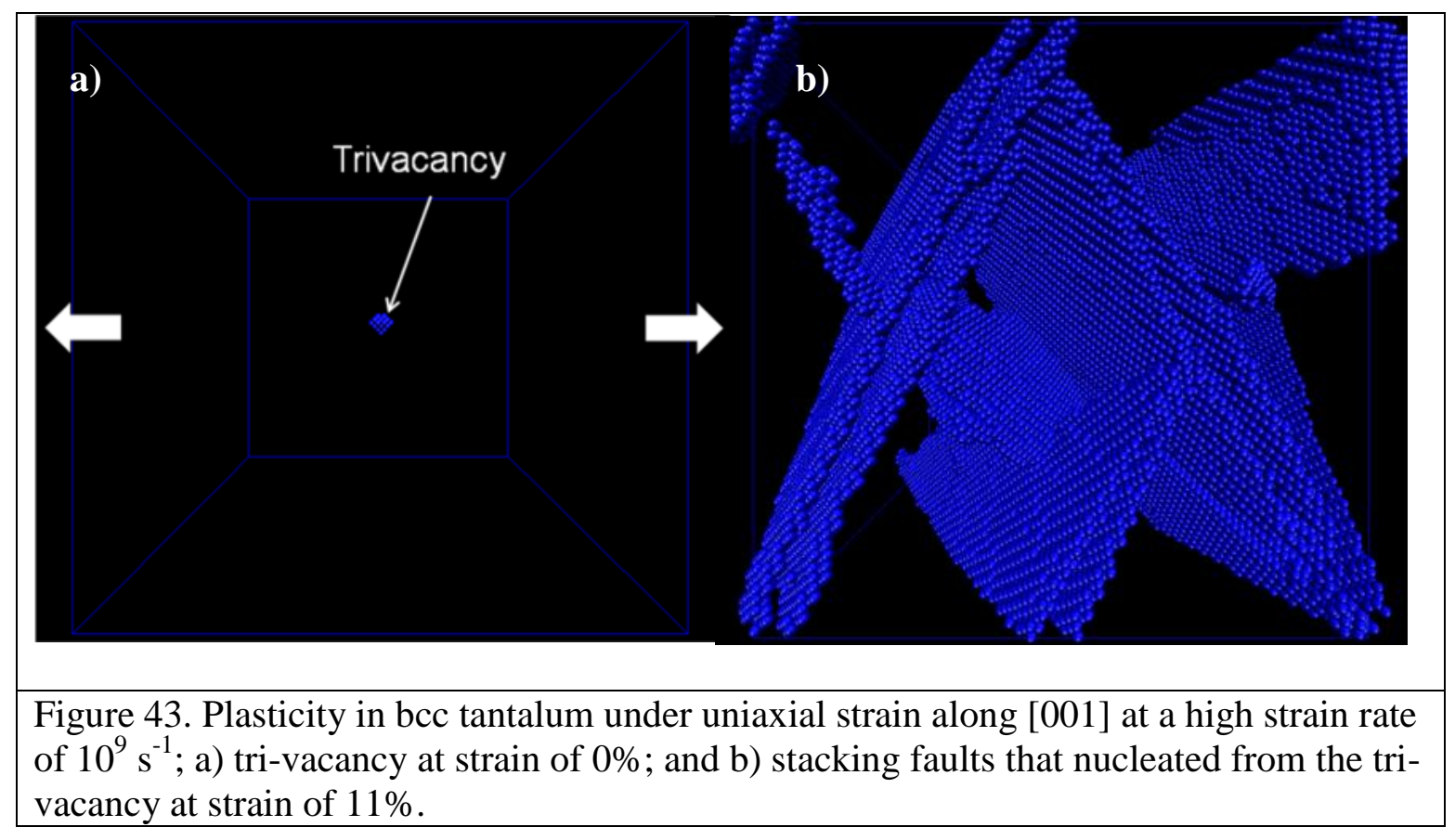




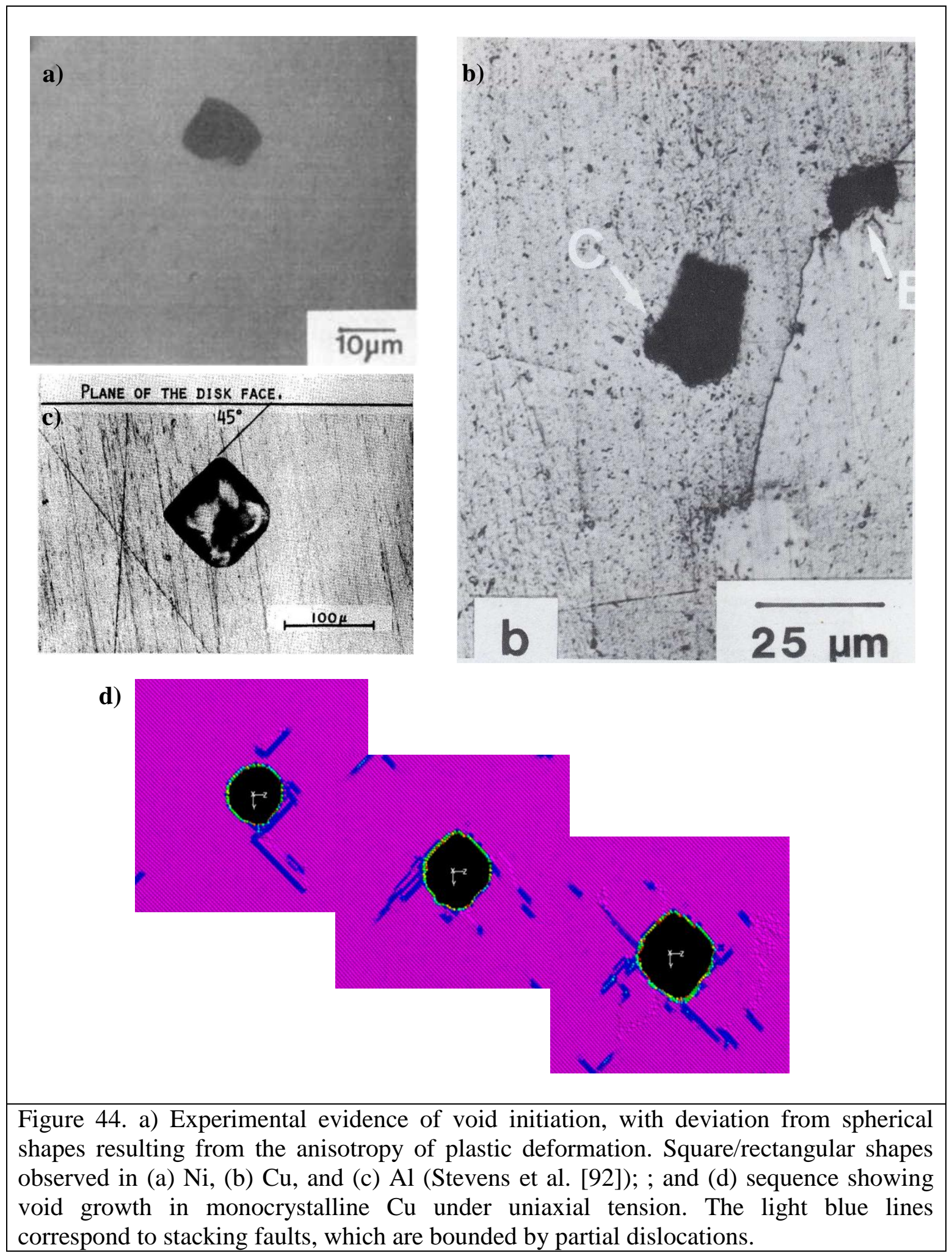



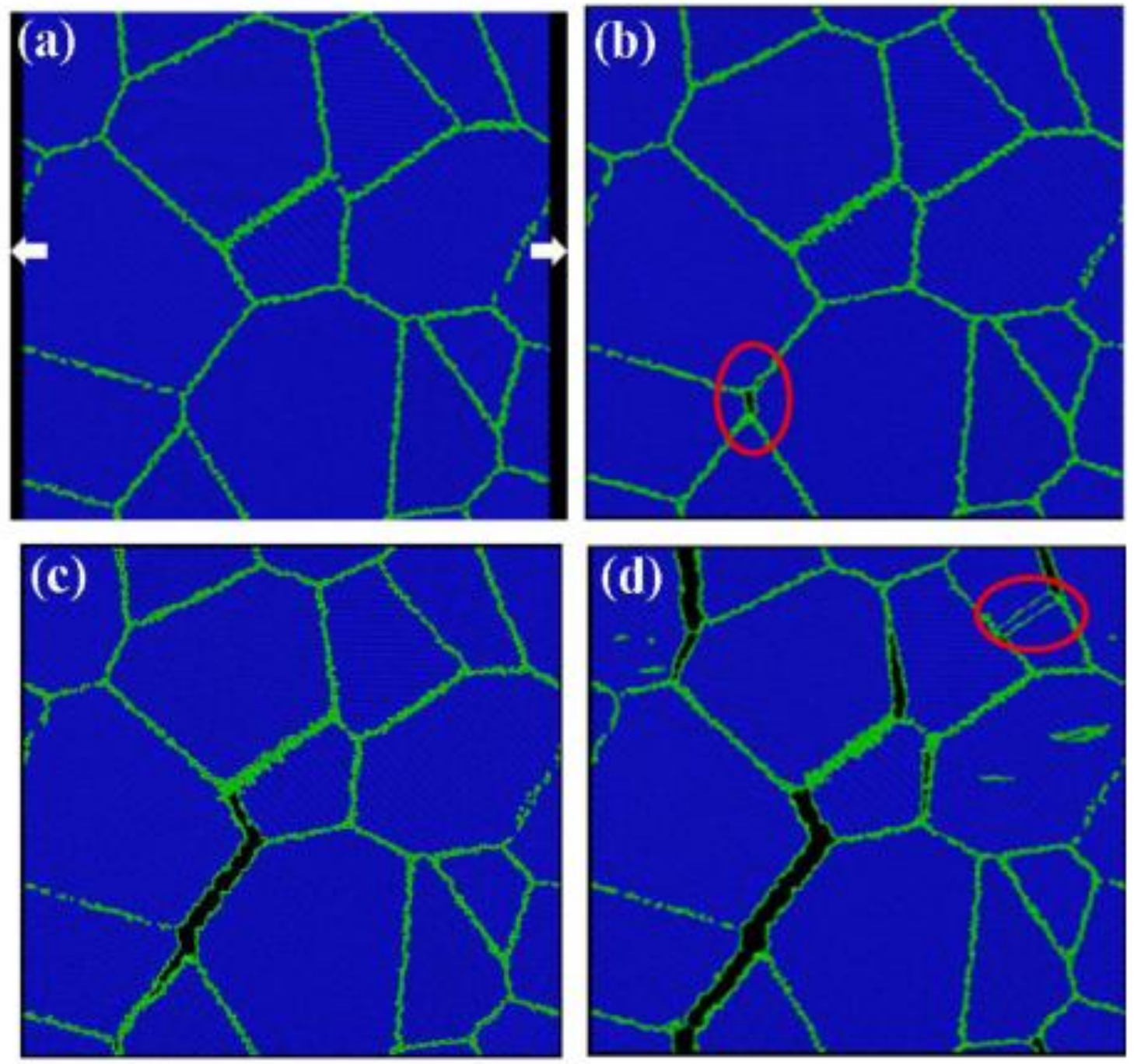

Figure 45. Simulation showing nanocrystalline Ta with an average grain size of $27 \mathrm{~nm}$, undergoing uniaxial tension; a) the arrows on the right and left signify the direction of tension; b) circled in red is the initiation of a void; c) void growth and coalescence leads to crack formation and propagation; and d) twinning is observed inside one of the grains, as circled in red. 


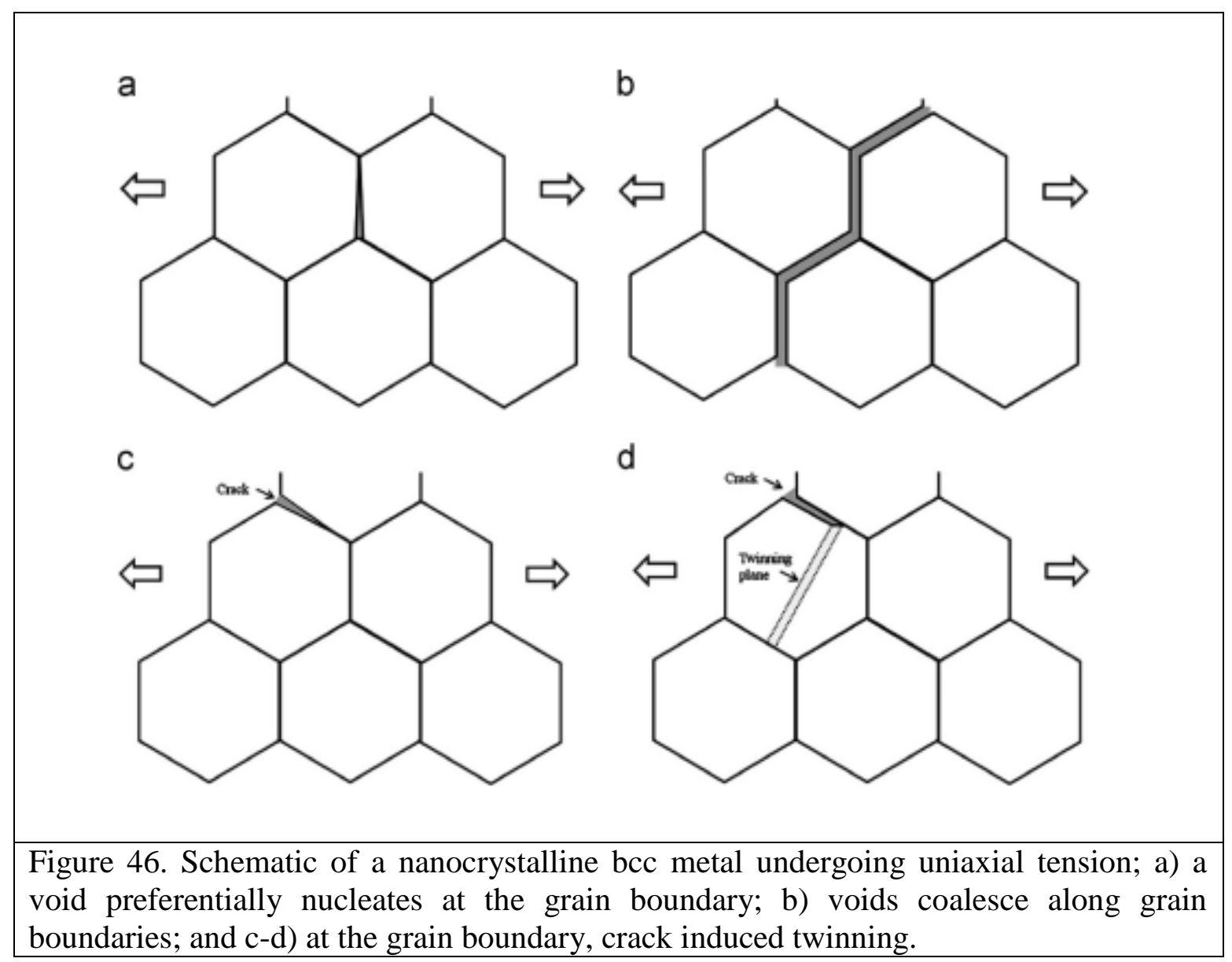




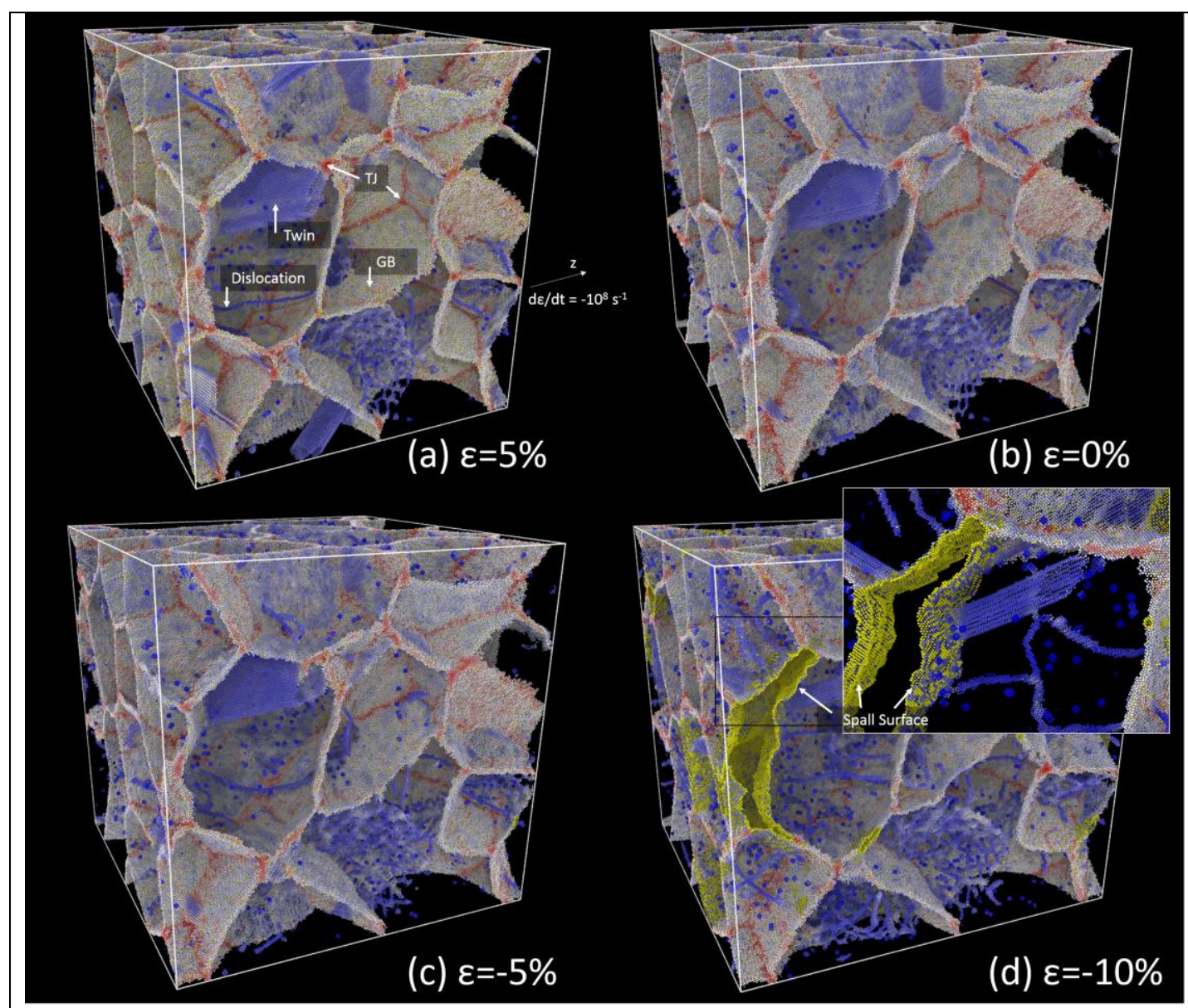

Figure 47. MD sequence showing spalling in nanocrystalline tantalum. Notice plastic deformation around growing voids, in contrast with situation in Fig. 45 and 46, where tension is applied uniformly on a nanocrystalline specimen without defects. Quasiisentropic compression followed by tension. The compressive strain rate is $10^{9} \mathrm{~s}^{-1}$ and tensile strain rate is $10^{8} \mathrm{~s}^{-1}$. Pre-compression leads to much more realistic grain boundary and defect structures. This in turn dictates that tensile failure is much more ductile including multiple dislocation and twin emissions that can be seen in (d), magnified in the inset. Adapted from Hahn et al. [81]. 


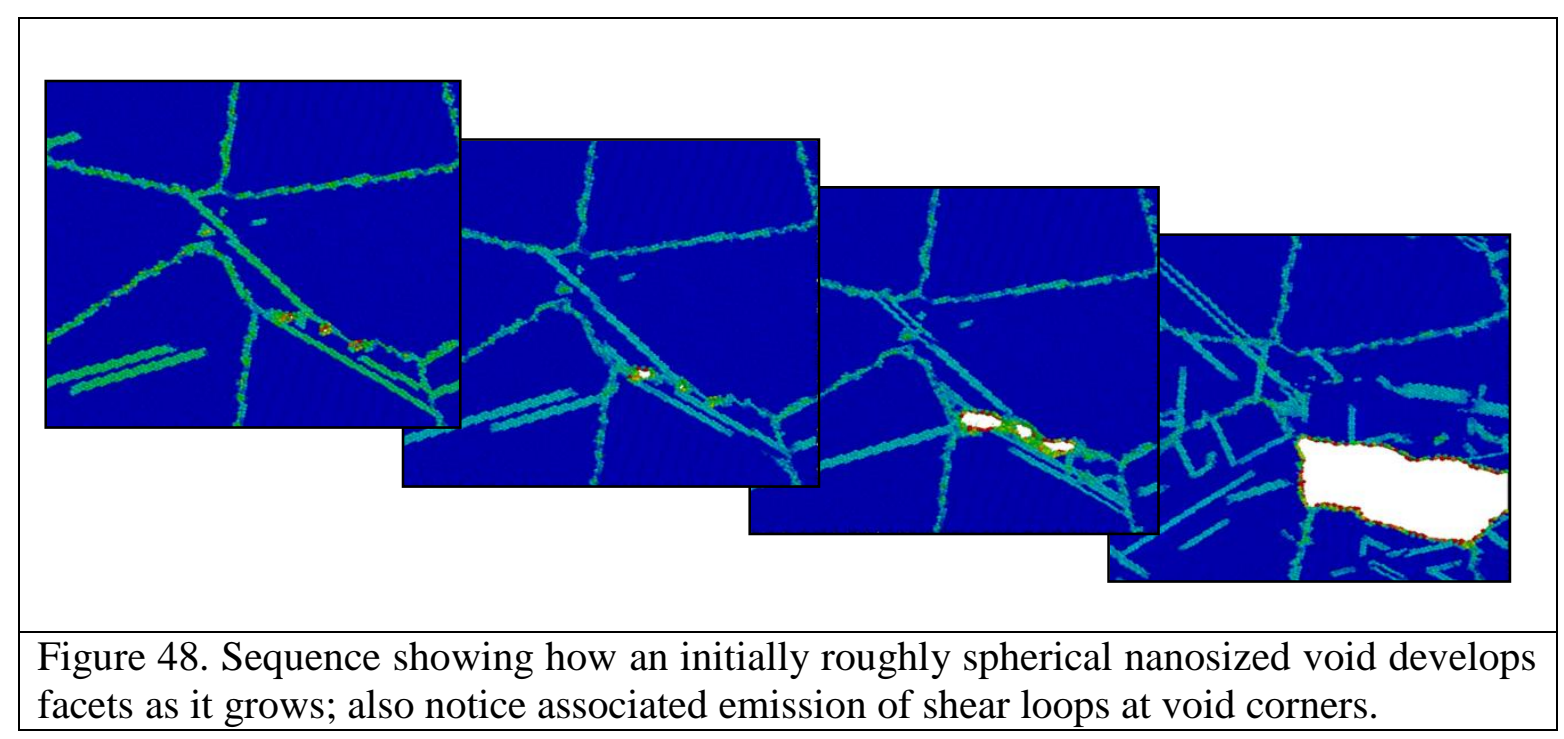



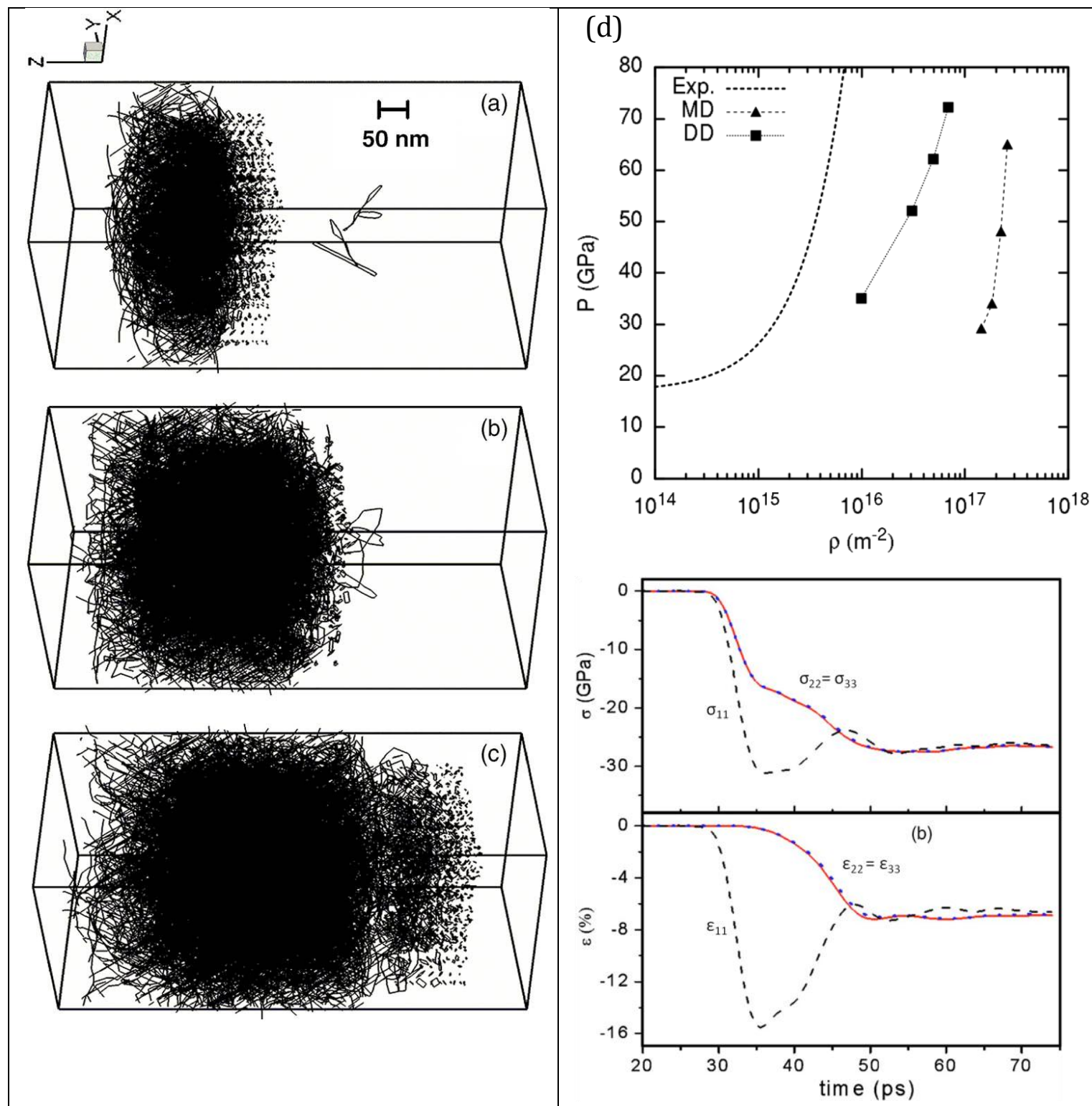

(e)

Figure 49. (a-c) Snapshots generated by dislocation dynamics (DD) simulations in copper subjected to shock compression at times of 67, 90, and 120 ps. Pressure of 35 $\mathrm{GPa}, 50 \mathrm{ps}$ rise time shock wave. Loops nucleate homogeneously as the wave travels through copper from left to right, while those previously nucleated grow as the crystal relaxes.(d) dislocation density as a function of pressure from DD and MD simulations compared to experimental results. (e) stresses and strains as a function of time obtained from DD; notice that stress state relaxes to hydrostatic as dislocations are nucleated and move. Adapted from Shehadeh et al. [98]. 


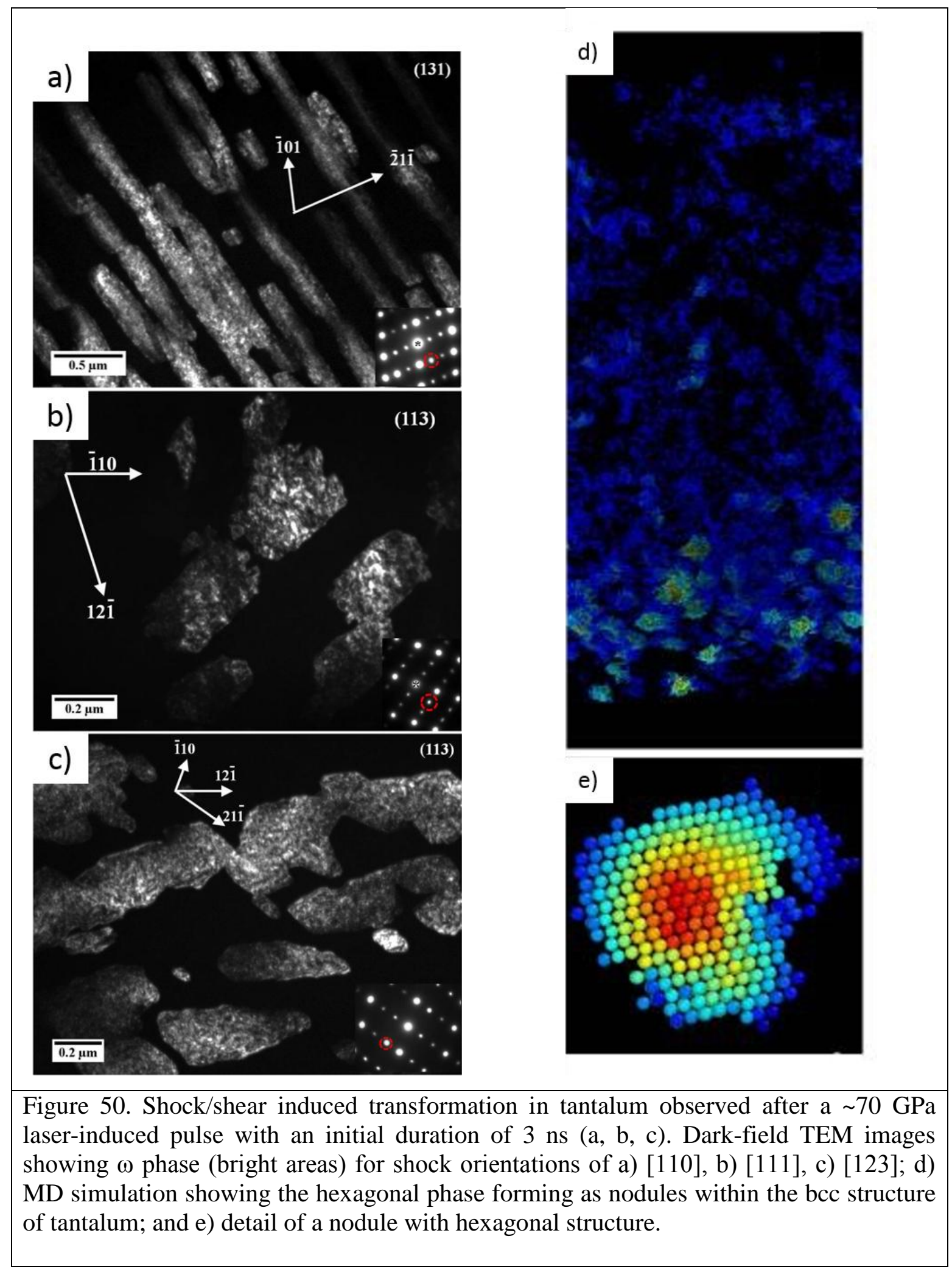




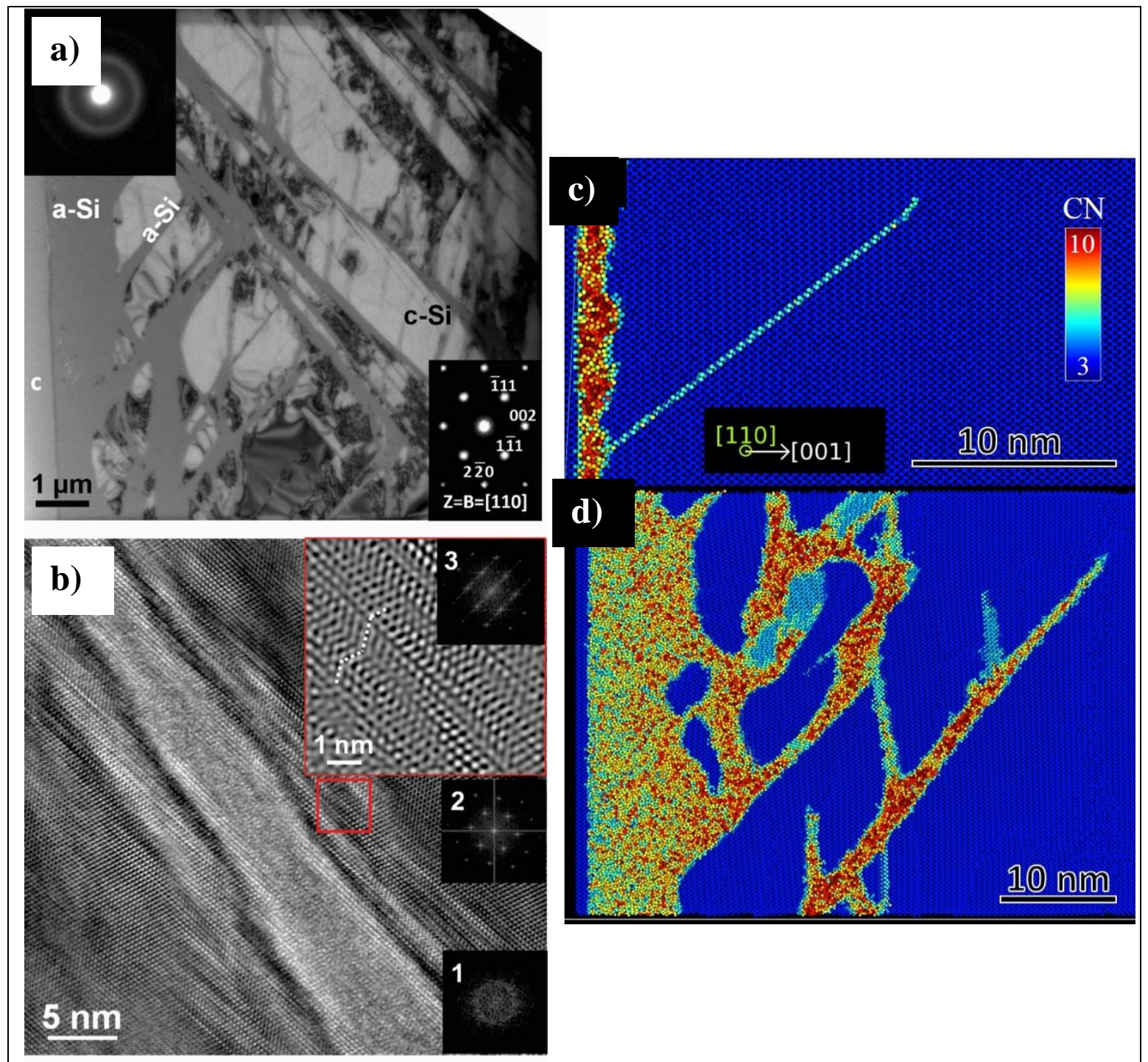

Figure 51. (a,b) Shock and shear-induced amorphization in silicon observed after pulsed laser compression at the Omega Facility (LLE). C-Si designates crystalline Si whereas a$\mathrm{Si}$ indicates the amorphous phase; and (c,d) MD simulations showing both the pressureinduced layer close to the energy deposition surface and the shear induced amorphization on right. From Zhao et al. [110]. 